DOE/BC/95000129

Distribution Category UC-122

Predicting the Transport Properties of

Sedimentary Rocks from Microgeometry

By

E.M. Schlueter

February 1995

Work Performed Under Contract No. DE-AC03-76SF00098

Prepared for

U.S. Department of Energy

Assistant Secretary for Fossil Energy

Robert Lemmon, Project Manager

Bartlesville Project Office

P.O. Box 1398

Bartlesville, OK 74005

Prepared by

Department of Materials Science and Mineral Engineering University of California and

Earth Sciences Division

Lawrence Berkeley Laboratory

University of California

Berkeley, CA 94720

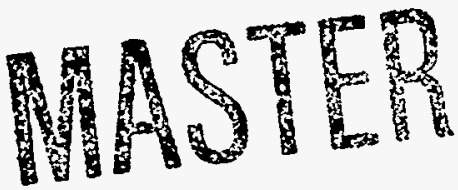

ACTBZUTION OF THIS DOCUMENT IS UNL NMTER 


\section{DISCLAIMER}

This report was prepared as an account of work sponsored by an agency of the United States Government. Neither the United States Government nor any agency thereof, nor any of their employees, make any warranty, express or implied, or assumes any legal liability or responsibility for the accuracy, completeness, or usefulness of any information, apparatus, product, or process disclosed, or represents that its use would not infringe privately owned rights. Reference herein to any specific commercial product, process, or service by trade name, trademark, manufacturer, or otherwise does not necessarily constitute or imply its endorsement, recommendation, or favoring by the United States Government or any agency thereof. The views and opinions of authors expressed herein do not necessarily state or reflect those of the United States Government or any agency thereof. 


\section{DISCLAIMER}

Portions of this document may be illegible in electronic image products. Images are produced from the best available original document. 


\section{Contents}

I. INTRODUCTION 2

II. OBJECTIVES 3

III. PREDICTING THE PERMEABILITY OF SEDIMENTARY ROCKS FROM MICROGEOMETRY

A. Effective medium theory $\ldots \ldots \ldots \ldots \ldots \ldots \ldots \ldots \ldots \ldots$

B. Method of analysis ..................... 6

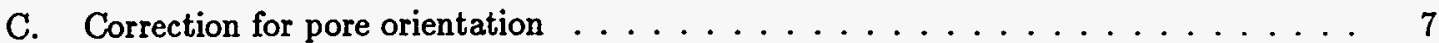

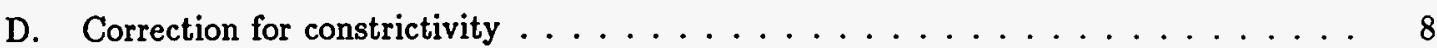

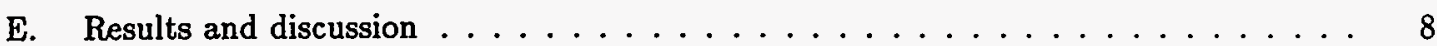

IV. PERIMETER-AREA POWER-LAW RELATIONSHIP OF PORES IN SEDIMENTARY ROCKS AND IMPLICATIONS FOR PERMEABILITY 16

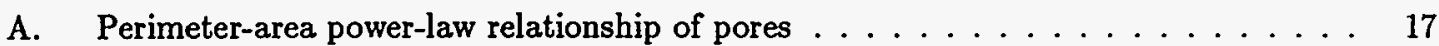

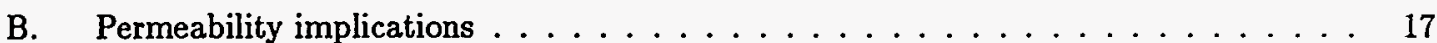

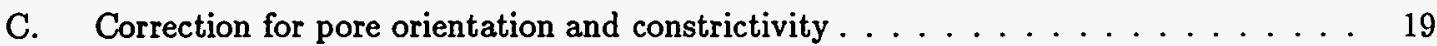

D. Results and discussion . . . . . . . . . . . . . . . . . . . . 19

V. PREDICTING THE ELECTRICAL CONDUCTIVITY OF SEDIMENTARY ROCKS FROM MICROGEOMETRY 27

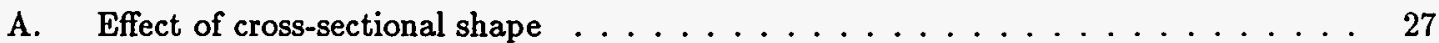

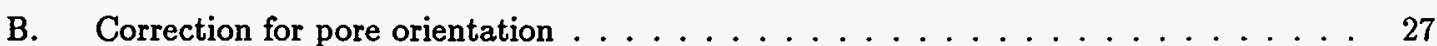

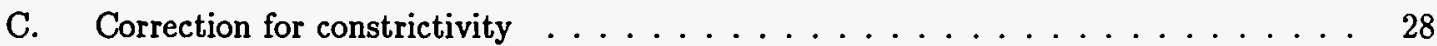

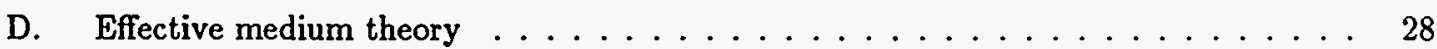

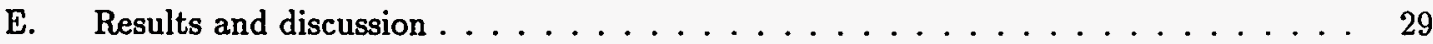

VI. FORMATION FACTOR AND THE MICROSCOPIC DISTRIBUTION OF WETTING PHASE IN PORE SPACE OF BEREA SANDSTONE

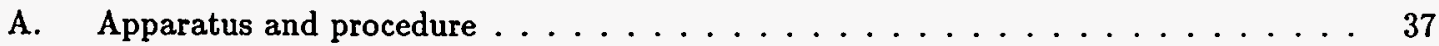

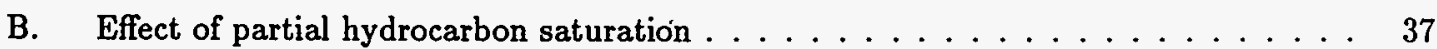

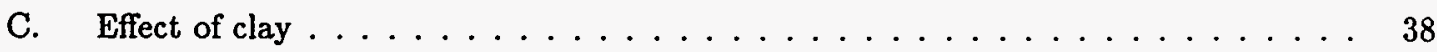

D. Effect of surface conductance $\ldots \ldots \ldots \ldots \ldots \ldots \ldots$

iii 
E. Effect of pore structure and topology $\ldots \ldots \ldots \ldots \ldots \ldots \ldots \ldots$

F. The Archie saturation exponent $\ldots \ldots \ldots \ldots \ldots \ldots \ldots$

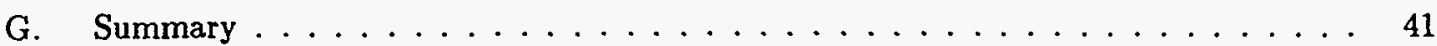

VII. PREDICTING THE CAPILLARY PRESSURE OF BEREA SANDSTONE FROM MICROGEOMETRY

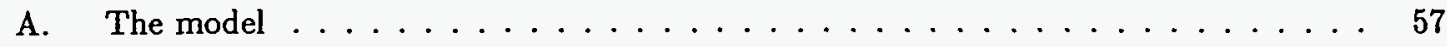

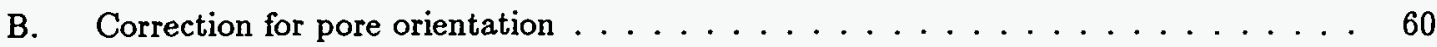

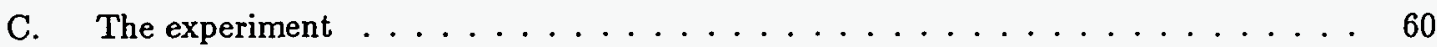

D. Effect of pore structure and topology $\ldots \ldots \ldots \ldots \ldots \ldots$

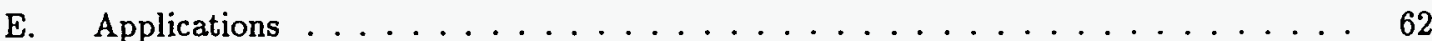

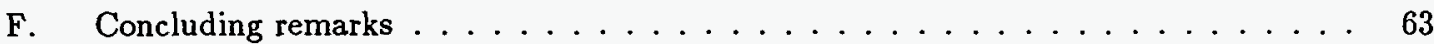

VIII. RECAPITULATION

$\begin{array}{ll}\text { IX. EXTENSIONS } & 84\end{array}$

$\begin{array}{ll}\text { X. ACKNOWLEDGMENTS } & 85\end{array}$ 


\section{List of Figures}

3.1 Stereo scanning electron micrograph of a Berea sandstone pore cast. The pore space is partially impregnated with Wood's metal alloy and the quartz grains removed by

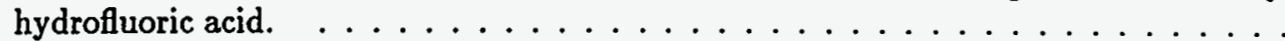

3.2 Typical scanning electron microscope photomicrograph of Berea sandstone. The rock is composed mainly of quartz grains (dark gray), feldspar grains (medium gray), and

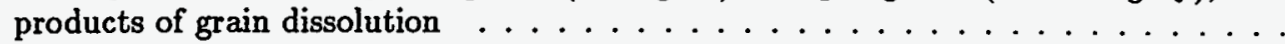

3.3 Pore-space contours obtained from computerized image analysis of the photomicrograph shown in Fig. $3.2 . \ldots \ldots \ldots \ldots \ldots$

3.4 Constriction factor for hydraulic flux, as function of the ratio of the minimum pore radius to the maximum pore radius of an individual pore. The calculated conductances of the pores must be $\ldots \ldots \ldots \ldots \ldots \ldots \ldots$

3.5 Schematic diagram of the 'skeleton' of a microscopically inhomogeneous pore system (after Doyen, 1988). . . . . . . . . . . . . . . . . . .

4.1 Schematic drawing of a rough walled tube (of radius $R_{o}$ ). Surface roughness does not have a strong effect on the overall fluid permeability of a tube, because a slightly smaller tube

4.2 Typical serial section of Saint-Gilles sandstone, at $100 \mathrm{X}$ magnification. The mineral grains of different shades are quartz, carbonate, feldspar, and muscovite, whereas the

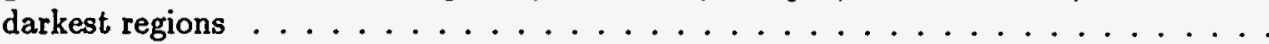

4.3 Pore-space contours obtained from serial section of Massilon sandstone (after Koplik et al., 1984) . . . . . . . . . . . . . . . . . . . . . . . . . . . . . .

4.4 Perimeter-area power-law relationship for Massilon sandstone. . . . . . . . . . .

4.5 Area frequency distribution of pores obtained from serial section of Massilon sandstone shown in Fig. 4.3. . . . . . . . . . . . . . . . . .

5.1 Typical scanning electron microscope photomicrograph of a Berea sandstone section. The rock is composed mainly of quartz grains (dark gray), feldspar grains (medium gray), and products of grain dissolution $\ldots \ldots \ldots \ldots$

5.2 Pore-space contours obtained from computerized image analysis of the section shown

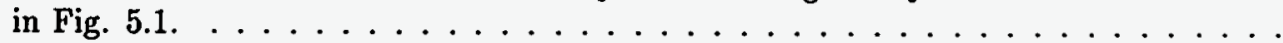

5.3 Constriction factor for electric flux, as functions of the ratio of the minimum pore radius to the maximum pore radius of an individual pore. The calculated conductances of the pores must be . . . . . . . . . . . . . . .

6.1 Schematic representation of the laboratory apparatus designed to simultaneously measure both electric and hydraulic conductivity. . . . . . . . . . . . . . . . 43

6.2 Experimental setup for one-dimensional paraffin impregnation. . . . . . . . . . 44

6.3 Effective formation factor vs. hydrocarbon paraffin saturation for Berea sandstone. The pore space was partially saturated with hydrocarbon paraffin. The remaining portion of the pore 
6.4 Scanning electron micrographs of a Berea sandstone specimen showing the presence of fine-grained clay minerals (mainly kaolinite) partly filling pores . . . . . . . . .

6.5 X-ray spectrum of clay minerals coating Berea sandstone sample pores shown in figure 6.4. EDX analysis yielding nearly equal peak heights of $\mathrm{Si}$ and $\mathrm{Al}$ confirms

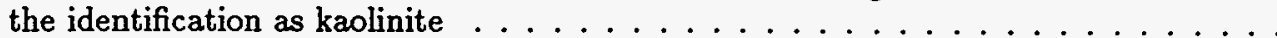

6.6 Conductivity data of Berea sandstone at different electrolyte concentrations. Zinc nitrate electrolyte solutions with concentrations ranging from $0.005 \mathrm{M}$ to $0.5 \mathrm{M}$ were used. . . . . . . . . . . . . . . . . . .

6.7 Mobility for kaolinite as a function of salt concentration and $\mathrm{pH}$ (after Williams and Williams, 1978).

6.8 Scanning electron micrograph collage of a Berea sandstone specimen impregnated with approximately $20 \%-30 \%$ paraffin. Actual width of field is about $6 \mathrm{~mm}$. The gray phase is quartz $\ldots \ldots \ldots \ldots \ldots \ldots \ldots$

6.9 Scanning electron micrograph collage of a Berea sandstone specimen impregnated with approximately $40 \%-50 \%$ paraffin. Actual width of field is about $6 \mathrm{~mm}$. The gray phase is quartz $\ldots \ldots \ldots \ldots \ldots \ldots \ldots$

6.10 Scanning electron micrograph collage of a Berea sandstone specimen impregnated with approximately $60 \%-70 \%$ paraffin. Actual width of field is about $6 \mathrm{~mm}$. The gray phase is quartz $\ldots \ldots \ldots \ldots \ldots \ldots \ldots$

6.11 Scanning electron micrograph collage of a Berea sandstone pore cast. Actual width of field is about $6 \mathrm{~mm}$. The rock pore space was completely filled with Wood's metal alloy and the quartz $\ldots \ldots \ldots \ldots \ldots \ldots \ldots \ldots$

6.12 Scanning electron micrograph collage of a Berea sandstone sample fully impregnated with Wood's metal alloy. Actual width of field is about $3 \mathrm{~mm}$. The gray phase is quartz grains and $\ldots \ldots \ldots \ldots \ldots \ldots \ldots \ldots \ldots \ldots \ldots \ldots \ldots \ldots$

6.13 Effective formation factor vs. electrolyte saturation for Berea sandstone (Case B). The pore space was partially saturated with hydrocarbon paraffin, with the remainder of

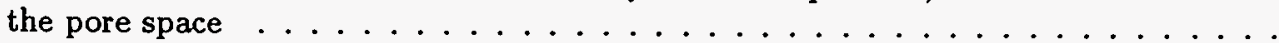

7.1 Experimental setup for three-dimensional Wood's metal imbibition. . . . . . . . 66

7.2 Experimental capillary pressure function of Berea sandstone. The rock has been imbibed with a nonwetting fluid (Wood's metal) at different equilibrium pressures and solidified in place. . . . . . . . . . . . . . . .

7.3 Experimental mercury porosimetry saturation curve of Berea sandstone (after Chatzis

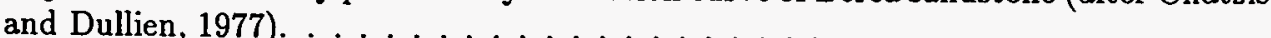

7.4 Top and bottom axial quarter sections of Berea sandstone core partially saturated with a nonwetting fluid (Wood's metal) at different equilibrium pressures, and solidified in place. . . . . . . . . . . . . . . . . . . .

7.5 Top section of Berea sandstone core partially saturated with a nonwetting fluid (Wood's metal) at an equilibrium pressure of $6.8 \mathrm{psia}$, and solidified in place. The procedure allows 
7.6 Top section of Berea sandstone core partially saturated with a nonwetting fluid (Wood's metal) at an equilibrium pressure of $6.9 \mathrm{psia}$, and solidified in place. The procedure allows $\ldots \ldots \ldots \ldots \ldots \ldots \ldots \ldots \ldots \ldots \ldots \ldots \ldots \ldots \ldots$

7.7 Top section of Berea sandstone core partially saturated with a nonwetting fluid (Wood's metal) at an equilibrium pressure of $7.2 \mathrm{psia}$, and solidified in place. The procedure allows $\ldots \ldots \ldots \ldots \ldots \ldots \ldots \ldots \ldots \ldots \ldots \ldots \ldots \ldots \ldots$

7.8 Scanning electron microscope photomicrograph collage of a 1 in $\times 1$ in Berea sandstone specimen partially saturated with approximately $50 \%$ Wood's metal at 8.5 psia equilibrium pressure $\ldots \ldots \ldots \ldots \ldots \ldots$

7.9 Scanning electron microscope photomicrograph collage of an enlarged partial section obtained from a Berea sandstone sample partially saturated with approximately $50 \%$ Wood's metal $\ldots \ldots \ldots \ldots \ldots \ldots \ldots \ldots \ldots \ldots \ldots \ldots \ldots$

7.10 Typical scanning electron microscope photomicrograph of Berea sandstone. The rock is composed mainly of quartz grains (dark-gray phase), feldspar grains (medium-gray phase), and products of grain dissolution $\ldots \ldots \ldots \ldots$

7.11 Computerized analysis of Berea sandstone image shown in Fig. 7.10 display intergranular pore space contours from which hydraulic radius distribution and transport

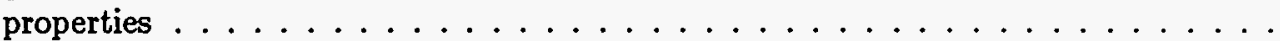

7.12 Perimeter-area power-law relationship of Berea sandstone obtained from pore space contours shown in previous figure. $\ldots \ldots \ldots \ldots \ldots$

7.13 Pore hydraulic radii frequency distribution of Berea sandstone obtained from pore space contours shown in Fig. $7.11 . \ldots \ldots \ldots \ldots$

7.14 Experimental vs. predicted capillary pressure function of Berea sandstone. To obtain the experimental capillary pressure function, the rock has been imbibed with a nonwetting fluid $\ldots \ldots \ldots \ldots \ldots \ldots \ldots \ldots \ldots \ldots \ldots \ldots \ldots \ldots$

7.15 Pore size distribution representative of a consolidated porous medium (Berea sandstone). Pore-throats are smaller than pore-bodies; $r_{t_{m}}=11 \mu \mathrm{m}, r_{b_{m}}=38 \mu \mathrm{m} \ldots \ldots$ 
$\ldots$ 


\section{List of Tables}

3.1 Intrinsic permeability of four sedimentary rocks. $\ldots \ldots \ldots \ldots \ldots$

4.1 Perimeter-area power-law relationship $\gamma$ parameter, and correlation coefficient $r$ measured from perimeter-area data of five sedimentary rocks. . . . . . . . . . . 26

5.1 Formation factor of three sedimentary rocks. $\ldots \ldots \ldots \ldots \ldots \ldots$

6.1 Formation Factor Data - Berea Sandstone, with a $0.5 \mathrm{M}$ zinc nitrate solution used as

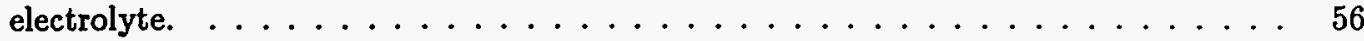

6.2 Resistivity Data - Berea Sandstone, with zinc nitrate solution saturating the sample. 56

7.1 List of comparative values to show equivalence of the reciprocal hydraulic radius $\left(1 / R_{H}\right)$ and the reciprocal mean radius of curvature $\left(1 / r_{1}+1 / r_{2}\right)$ in a capillary . 81 


\title{
Predicting the Transport Properties of Sedimentary Rocks from Microgeometry*
}

\author{
E. M. Schlueter \\ Department of Materials Science and Mineral Engineering \\ University of California \\ Berkeley, California 94720 \\ and \\ Earth Sciences Division \\ Lawrence Berkeley Laboratory \\ University of California
}

\begin{abstract}
We investigate through analysis and experiment how pore geometry, topology, and the physics and chemistry of mineral-fluid and fluid-fluid interactions affect the flow of fluids through consolidated/partially consolidated porous media. Our approach is to measure fluid permeability and electrical conductivity of rock samples using single and multiple fluid phases that can be frozen in place (wetting and nonwetting) over a range of pore pressures. These experiments are analyzed in terms of the microphysics and microchemistry of the processes involved to provide a theoretical basis for the macroscopic constitutive relationships between fluid-flow and geophysical properties that we develop. The purpose of these experiments and their analyses is to advance the understanding of the mechanisms and factors that control fluid transport in porous media. This understanding is important in characterizing porous media properties and heterogeneities before simulating and monitoring the progress of complex flow processes at the field scale in permeable media.
\end{abstract}

*This research was supported by the U.S. Department of Energy through the Assistant Secretary for Fossil Energy, Bartlesville Project Office, Advanced Extraction Process Technology (AEPT), under contract No. DE-AC22-89BC14475, with the University of California at Berkeley. Additional funding was provided by the Director, Office of Energy Research, Office of Basic Energy Sciences, Engineering and Geosciences Division, under Contract No. DE-AC03-76SF00098 with the Lawrence Berkeley Laboratory. 


\section{INTRODUCTION}

Determination of subsurface hydrologic parameters that characterize fluid flow through porous media (e.g., hydraulic conductivity, capillary pressure, and relative permeability) is crucial to the understanding, monitoring, and simulating of complex flow problems in permeable media, including multiphase, multicomponent field scale processes as well as waste remediation and/or isolation. Results of numerical simulations quantifying the effects of the release and transport of non-condensible gas and non-aqueous phase liquid (NAPL) contaminants in subsurface formations highlight the impact of macroscopic transport properties on system behavior (Schlueter et al., 1990; Falta et al., 1992). Unfortunately, a simulation is only as good as the underlying reservoir description, and therefore it depends heavily on the physical properties as defined. The macroscopic transport properties of porous (and fractured) media depend critically upon processes at the pore level, which are controlled by the geometry and connectivity of the pore space. Consequently, there is a need for a first-principle understanding of how pore morphology and other related factors can be used to predict basic porous media physical properties (hydraulic and electric) such as intrinsic permeability, formation factor, relative permeability, and capillary pressure.

Our approach is to study, through analysis and experiment how pore geometry, topology and the physics of mineral-fluid and fluid-fluid interactions affect both the flow of fluids through rocks and the relationship between the fluid transport properties and the electrical behavior and properties of the porous media under consideration. The theoretical analysis has been useful in defining and ranking needed experiments, and to successfully correlate measurements of important transport properties, such as permeability and electrical conductivity of consolidated/partially consolidated porous media, to the microgeometry of the pore space. On the other hand, the experimental work has provided valuable insights into the pore level complexity of the natural porous media. It is clear from this study that heterogeneity characterization at the scale of pores, conventional macroscopic flow theory, and fluid flow modeling will not only improve our understanding of the fluid dynamics in permeable media but also will enable us to better monitor, simulate, and predict complex flow processes at the field scale.

${ }^{1}$ Schlueter, E.M., and Pruess, K. 1990. Sensitivity studies on parameters affecting gas release from an underground rock cavern. Lawrence Berkeley Laboratory Report LBL-28818, Berkeley, Calif., January. 
${ }^{2}$ Falta, R.W., Pruess, K., Javandel, I., and Witherspoon, P.A. 1992. Numerical modeling of steam injection for the removal of nonaqueous phase liquids from the subsurface, 2, code validation and application. Water Resour. Res., 28 (2), February.

\section{OBJECTIVES}

The overall objectives of this study are:

- to study the microscopic geometry, topology and heterogeneity of the pore space of consolidated/partially consolidated rocks by direct observation, i.e. dissolution of the mineral phase, or sectioning and polishing of the specimen for study in scanning electron and optical microscopes;

- to measure fluid permeability, electrical conductivity, and capillary pressure of rocks using multiple fluid phases that can be frozen in place to allow direct visualization and analysis of wetting and non-wetting fluid distributions in the pore space;

- to compare hydraulic and electrical conductivity, and capillary pressure data to existing data on rocks (if available) obtained using conventional methods;

- to evaluate hydraulic and electrical conductivity experimental data in terms of microphysics and microchemistry of processes involved (i.e., effect of clay coating pores);

- to examine hydraulic and electrical conductivity experimental data in terms of the role of the pore structure and heterogeneities on the observed fluid distributions at each saturation;

- to understand the relationship of pore microstructure and heterogeneity to macroscopic hydraulic and electrical conductivities for consolidated/partially consolidated porous materials;

- to study the experimental data and fluid distributions of rocks at low wetting and nonwetting fluid saturations and near threshold saturation conditions;

- to develop relations between pore microstructure and macroscopic single and multiphase transport properties of rocks using appropiate statistical and network techniques as well as fractal distributions and percolation theory; 
- to ultimately use true macroscopic transport properties to better understand, simulate, and monitor large scale transport processes in permeable media.

The following sections (III-VII) provide examples of results up to date (November, 1992) from the author's doctoral research at the University of California at Berkeley (Schlueter E.M., 1993).

${ }^{1}$ Schlueter, E.M. 1993. Predicting the transport properties of sedimentary rocks from microgeometry. Doctoral Dissertation, Univ. of California at Berkeley, in progress.

\section{PREDICTING THE PERMEABILITY OF SEDIMENTARY ROCKS FROM MICROGEOMETRY}

The macroscopic transport properties of porous and fractured media depend critically upon processes at the pore level, which are very dependent on the geometry and connectivity of the pore space. The primary focus of this research is to understand, through analysis and experiment, how fluids in pores affect the hydraulic properties of rocks, and to develop equations relating these macroscopic properties to the microscopic geometry and structure of the pore space. It has been our aim to assemble a comprehensive picture of a rock based on a geologically sound and physically accurate framework. The theoretical analysis has been useful in defining and ranking needed experiments, and has been able to successfully correlate measurements of important transport properties such as intrinsic permeability of sandstone to the microgeometry of the pore space.

In this study, 2-D scanning electron microscope (SEM) photomicrographs of rock cross-sections have been employed to infer the hydraulic conductance of the individual pores. We assume that the pores are cylindrical tubes of varying radius, and that they are arranged on a cubic lattice, so that the coordination number of the network is 6 . The hydraulic conductance of each tube is estimated from its area and perimeter, using the hydraulic radius approximation and the Hagen-Poiseuille equation. In the section under consideration, the pore cross-sections are assumed to be randomly oriented with respect to the directions of the channel axes. The orientation effect has been corrected by means of geometrical and stereological considerations. Account is also taken for possible variation of the cross-sectional area along the length of each tube, e.g., pore necks and bulges. The effective medium theory of solid state physics is then used to replace each individual conductance with 
an effective average conductance. Finally, a unit cubic cell is extended to relate the effective hydraulic tube conductances to the continuum values of permeability. Preliminary results, using Berea, Boise, Massilon, and Saint-Gilles sandstone, yield very close agreement between the predicted and measured permeabilities, with essentially no arbitrary parameters in the model.

\section{A. Effective medium theory}

Consider an inhomogeneous, disordered, continuous composite system (conductive/non conductive) in which one can define locally a given conductive property, e.g., hydraulic conductance, which can be calculated from the geometry of the conductive element (e.g., the coefficient in Poiseuille's law for cylindrical tubes). This is possible as long as the dimensions of the local elements are large with respect to the scale of the conduction process involved. According to Kirkpatrick (1973), such a medium can be approximated by a 3-D network with the same topology in which all the conductances have a single effective value. The effective medium can be defined as one in which the macroscopic conductance is the same as for the heterogeneous system, and therefore the effective conductance can be considered as the mean value controlling the physical property of concern. Since we are concerned with a random medium, it is assumed that no spatial correlation exists between the individual conductances. The theory leads to the requirement that the perturbation introduced when each individual conductance is replaced by the effective conductance averages to zero. The resulting implicit equation defining the effective conductance is

$$
\left\langle\frac{c_{e f f}-c_{i}}{[z / 2-1] c_{e f f}+c_{i}}\right\rangle=0
$$

where the angle brackets are for the average with respect to the probability distribution function $p(c)$ of the individual conductances $c_{i}$, and $z$ is the average coordination number of the network (the number of branches meeting at a node). The effective medium theory coupled with a network of resistors has been used by Koplik et al. (1984) to predict permeability of Massilon sandstone, although the predicted value was ten times higher than the measured value. Doyen (1988) calculated the transport properties of Fontainebleau sandstone, and predicted permeability within a factor of three of the measured value. These models do not account for the fact that the 2-D section under consideration slices each pore at a random angle to its axis, nor for the variation of the cross-sectional area along the length of each tube, both of which are significant effects. 


\section{B. Method of analysis}

The concept of permeability allows a macroscopic description of the fluid flow phenomena in porous media under a regime of sufficiently low fluid velocities (Scheidegger 1974). This property is linked to other properties of porous media such as capillary pressure and relative permeability. In order to understand the relationships, one has to understand how all those properties are conditioned by the connectivity and geometrical properties of the pore space. The simplest model that can be constructed is one representing a porous medium by a bundle of straight, parallel cylindrical capillaries of uniform diameter that go from one face of the porous medium to the other. Equations based on these type of 1-D model are called the Kozeny equations. The opposite extreme of this parallel case would be to assume a serial model in which all the pores are connected in series. Obviously, this model is as unrealistic as the parallel model, and a realistic model lies between these extremes.

A SEM stereo photomicrograph taken from a pore cast of Berea sandstone pore space is presented in Fig. 3.1. As revealed, the pore space is composed of a 3-D irregular network of irregularly shaped pores. Although an exact description of key pore space morphological characteristics is rather complex, it is possible to isolate three main features: (1) multiple connectivity of pore segments, (2) converging-diverging cross-section of pores, and (3) roughness and irregularity of the pore wall.

Since the actual rock geometry is too complex for any quantitative study, we have replaced it by a standard model geometry that preserves the main observed morphological features. The local conductive elements have been obtained from 2-D SEM micrographs of Berea, Boise, Massilon, and Saint-Gilles sandstone (Fig. 3.2). Typical pore-space contours obtained from serial sections are shown in Fig. 3.3. The hydraulic conductance of each tube of varying radius is estimated from its area and perimeter, using the hydraulic radius approximation and Poiseuille's law, while the electrical conductance is related only to the cross-sectional area of the tube.

According to the Hagen-Poiseuille equation, the volumetric flux of fluid through a cylindrical tube of radius $\mathrm{r}$ is

$$
q=\frac{\pi r^{4}}{8 \mu} \nabla P
$$

where $\mu$ is the absolute viscosity, and $\nabla P$ is the pressure gradient. We now use the hydraulic radius concept to rewrite Eq. 2 in a form that is applicable to noncircular pores. (As part of our analysis of the relationships between pore structure and transport properties, we have studied the effect of pore shape on permeability using the mathematical analogy between fluid flow through a cylindrical tube and elestic torsion of a cylindrical rod (Berker R., 1963). The results of this study show that the 
error involved in the hydraulic radius approximation lies well within $\pm 30 \%$.) We have therefore concluded that the conductivity of a tubular pore is well approximated by the hydraulic radius theory. The hydraulic radius $R_{H}$ of the tube is defined as

$$
R_{H}=\frac{\text { area }}{\text { wetted perimeter }}=\frac{r}{2}
$$

Using Eq. 3, we can rewrite Eq. 2 as

$$
q=\frac{R_{H}^{2} A}{2 \mu} \nabla P
$$

where $A$ is the area of the tube. The hydraulic conductance per unit length of each tube is therefore given by

$$
C_{h}=\frac{R_{H}^{2}}{2} A
$$

\section{Correction for pore orientation}

In the 2-D section under consideration, however, the pore cross-sections are randomly oriented with respect to the directions of the channel axes. The orientation effect has been corrected by means of the following geometrical and stereological considerations, which are exact for the case of circular cross-sections:

$$
\left(R_{H}^{2} A\right)_{a c t u a l}=\frac{1}{2}\left\langle\frac{1}{\cos \theta\left(1+\cos ^{2} \theta\right)}\right\rangle^{-1}\left(R_{H}^{2} A\right)_{\text {measured }}
$$

where the angle bracketed expression is the spherical average for pores of random orientation:

$$
\left\langle\frac{1}{\cos \theta\left(1+\cos ^{2} \theta\right)}\right\rangle=\frac{\int_{0}^{\pi} \int_{0}^{\theta_{\max }} \frac{\sin \theta d \theta d \phi}{\cos \theta\left(1+\cos ^{2} \theta\right)}}{\int_{0}^{\theta_{\max }} \sin \theta d \theta d \phi}
$$

with $\theta_{\max }=\arctan (L / D)$, and $L / D$ being the maximum ratio of pore length to diameter. Using an average value of $L / D=5$, as estimated from the micrographs, we find that

$$
\left(R_{H}^{2} A\right)_{\text {actual }}=0.40\left(R_{H}^{2} A\right)_{\text {measured }}
$$

(Evualation of the integral appearing in Eq. 7 shows that the factor 0.40 appearing in Eq. 8 is very insensitive to the value chosen for $L / D$ ). 


\section{Correction for constrictivity}

In addition, constrictions within the individual branch channels, i.e., pore necks and bulges, have been taken into account using an analysis based on a sinusoidal variation of cross-section. In the permeability analysis, for example, the factor accounts for the ratio of $\left\langle R^{-4}\right\rangle^{-1}$, which governs the conductance of a tube of varying radius, to $\langle R\rangle^{4}$, which is the value estimated from the micrographs. We have estimated a throat-to-pore radius ratio of 0.5 from a pore cast of Berea sandstone, and tentatively use this value for each rock. Figure 3.4 then indicates an hydraulic constriction factor of 0.55 . Although some sandstones also exhibit roughness at scales much smaller than the average pore diameter, it is known that such roughness has little effect on the hydraulic conductance (Berryman and Blair, 1987), and can therefore be ignored. Study of SEM photomicrographs of Berea sandstone has indicated the presence of a statistically isotropic 3-D pore structure well represented by Fig. 3.5. These observations have led to the assumption that the pores of varying size are arranged on a cubic lattice, so that the coordination number of the network is 6 . The effective medium theory from solid state physics is then used to replace each individual conductance with an effective average conductance as follows. For a general discrete distribution of conductances, Eq. 1 takes the form,

$$
\sum_{i=1}^{N} \frac{c_{e f f}-c_{i}}{[z / 2-1] c_{e f f}+c_{i}}=0
$$

where the sum is over all $N$ conductive elements. For a coordination number of $z=6$, Eq. 9 has been solved numerically to allow for the calculation of the effective medium conductance, given the individual conductances.

Finally, a unit cubic cell is extended to relate the effective hydraulic tube conductance to the continuum values of permeability by means of the following expression,

$$
k=\frac{N C_{e f f}}{A_{p h} \tau}
$$

where $C_{\text {eff }}$ is the effective conductance of the cubic network of coordination number $6, N$ the number of pore elements in the micrograph, $A_{p h}$ the area of the photograph, and $\tau$ the tortuosity of a cubic lattice, which is 3 .

\section{E. Results and discussion}

Preliminary results are presented in Table 3.1. Good agreement was found between measured and predicted hydraulic conductivity, for a variety of sandstones when using the cubic lattice model, with essentially no arbitrary adjustable parameters. It should 
be noted that if a Kozeny-type parallel $(z=\infty)$ or a serial model $(z=2)$ is applied instead, permeability is overpredicted or underpredicted by at least a factor of three.

${ }^{1}$ Berker, R. 1963. Integration des equations du mouvement d'un fluid visqueux incompressible. In Handbuch der Physik 8 (2), p.1-384. Berlin: Springer-Verlag.

${ }^{2}$ Berryman, J.G., and Blair, S.C. 1987. Kozeny-Carman relations and image processing methods for estimating Darcy's constant. J. Appl. Phys. 62 (6), p.2221-2228.

${ }^{3}$ Doyen, P. 1988. Permeability, conductivity, and the geometry of sandstone. J. Geophys. Res. 93 (B7), p.7729-7740.

${ }^{4}$ Kirkpatrick, S. 1973. Percolation and conduction. Rev. Mod. Phys. 45 (4), p.574587.

${ }^{5}$ Koplik, J., Lin, C., and Vermette, M. 1984. Conductivity and permeability from microgeometry. J. of Appl. Phys. 56 (11), p.3127-3131.

${ }^{6}$ Persoff, P. 1990. Private communication (Gaz de France. Departement Reservoirs Souterrains. Memorandum: M.D5 - LM/DT - G 1036, June 22 ${ }^{\text {nd }}, 1988$ ).

${ }^{7}$ Scheidegger, A.E. 1974. The physics of flow through porous media. University of Toronto Press. 


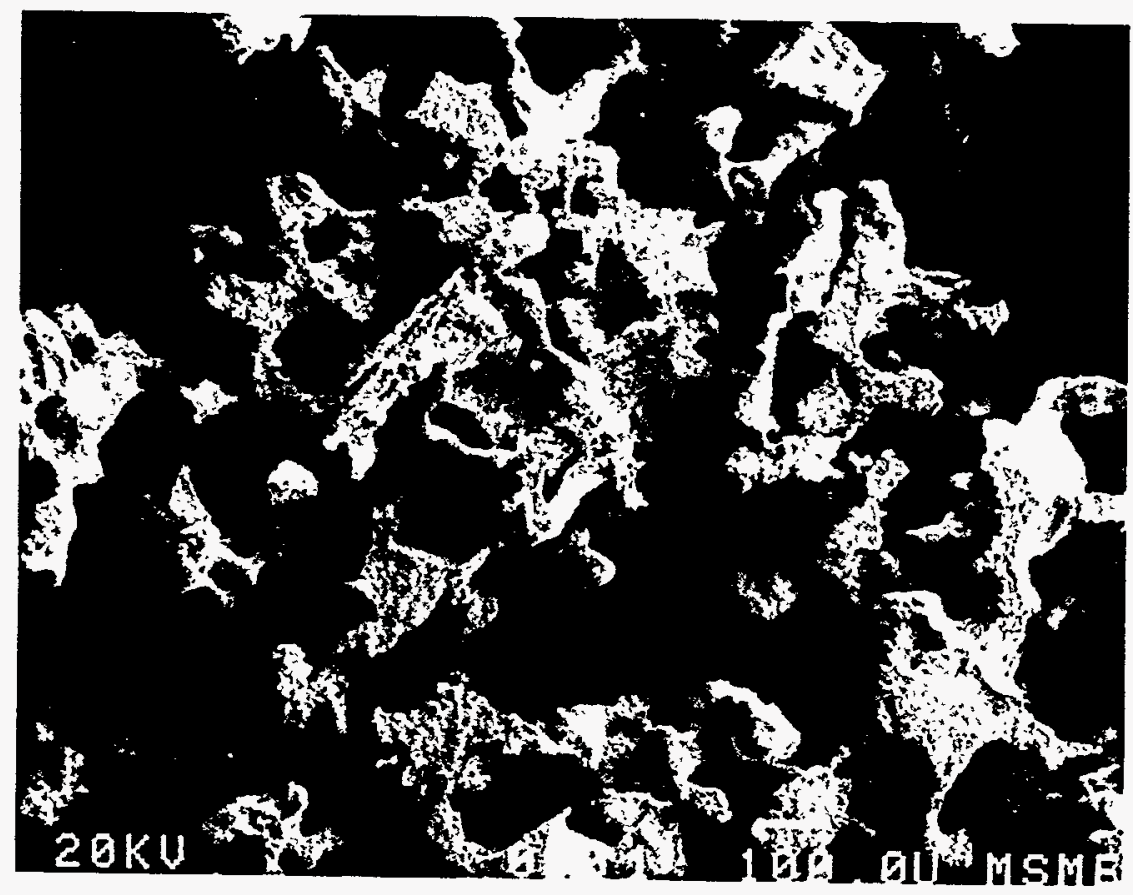

FIG. 3.1. Stereo scanning electron micrograph of a Berea sandstone pore cast. The pore space is partially impregnated with Wood's metal alloy and the quartz grains removed by hydrofluoric acid. Actual width of field is about $1 \mathrm{~mm}$. 


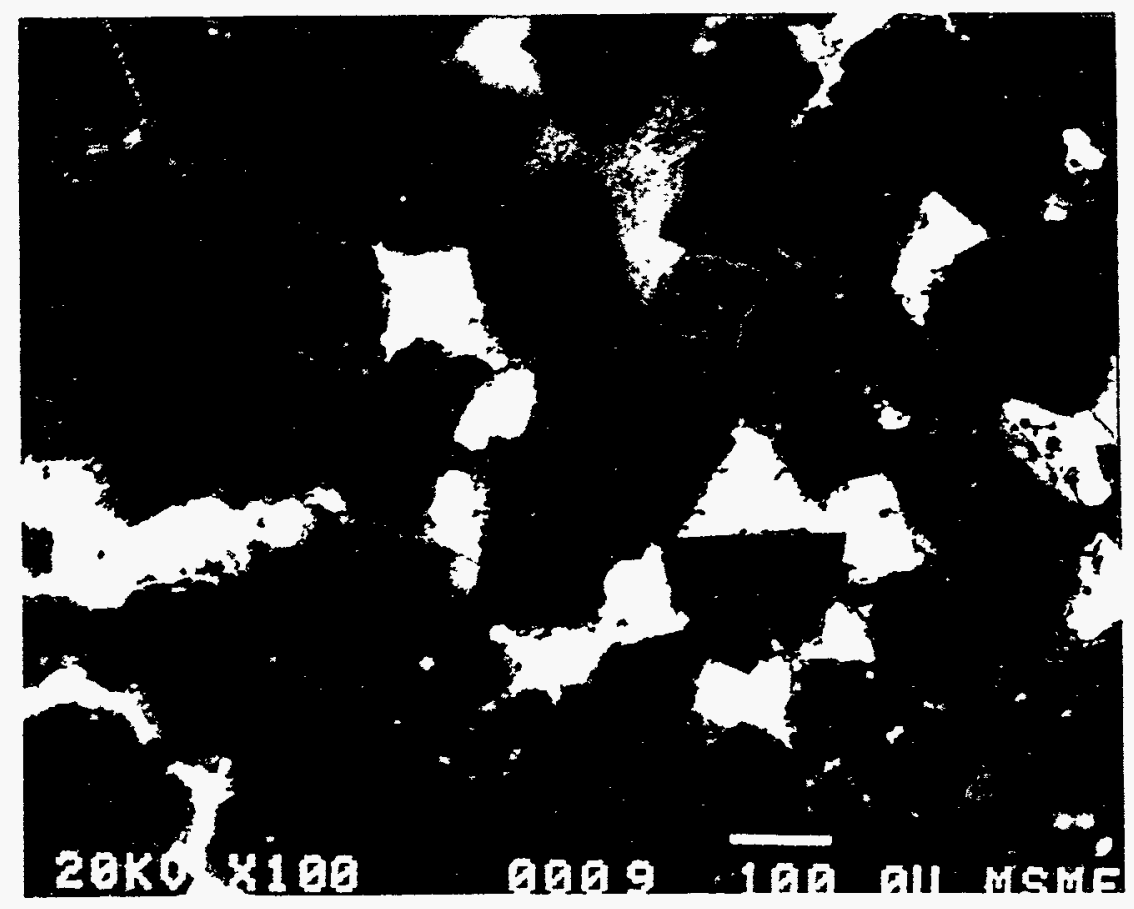

FIG. 3.2. Typical scanning electron microscope photomicrograph of Berea sandstone. The rock is composed mainly of quartz grains (dark gray), feldspar grains (medium gray), and products of grain dissolution (light gray). The pore space is impregnated with Wood's metal alloy (white), and epoxy (black). Actual width of field is about 1 $\mathrm{mm}$. 


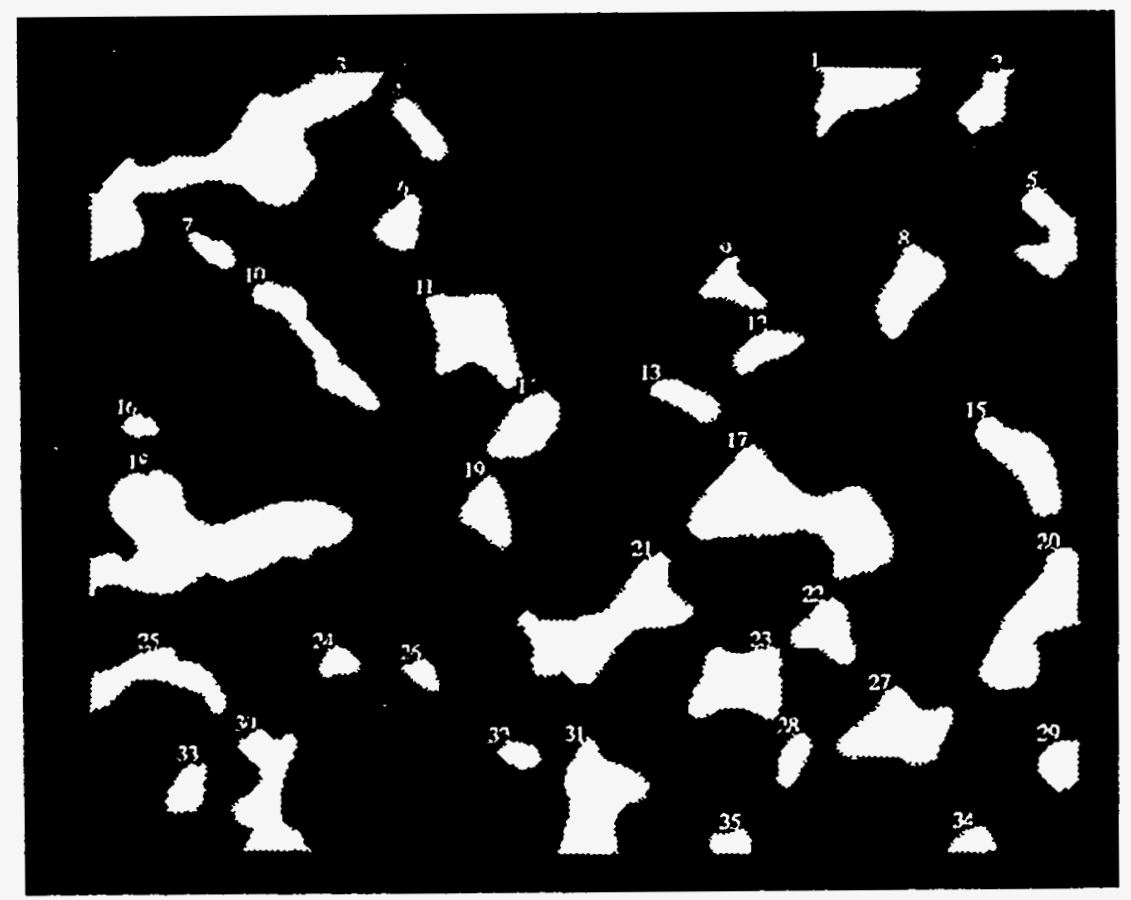

FIG. 3.3. Pore-space contours obtained from computerized image analysis of the photomicrograph shown in Fig. 3.2. 


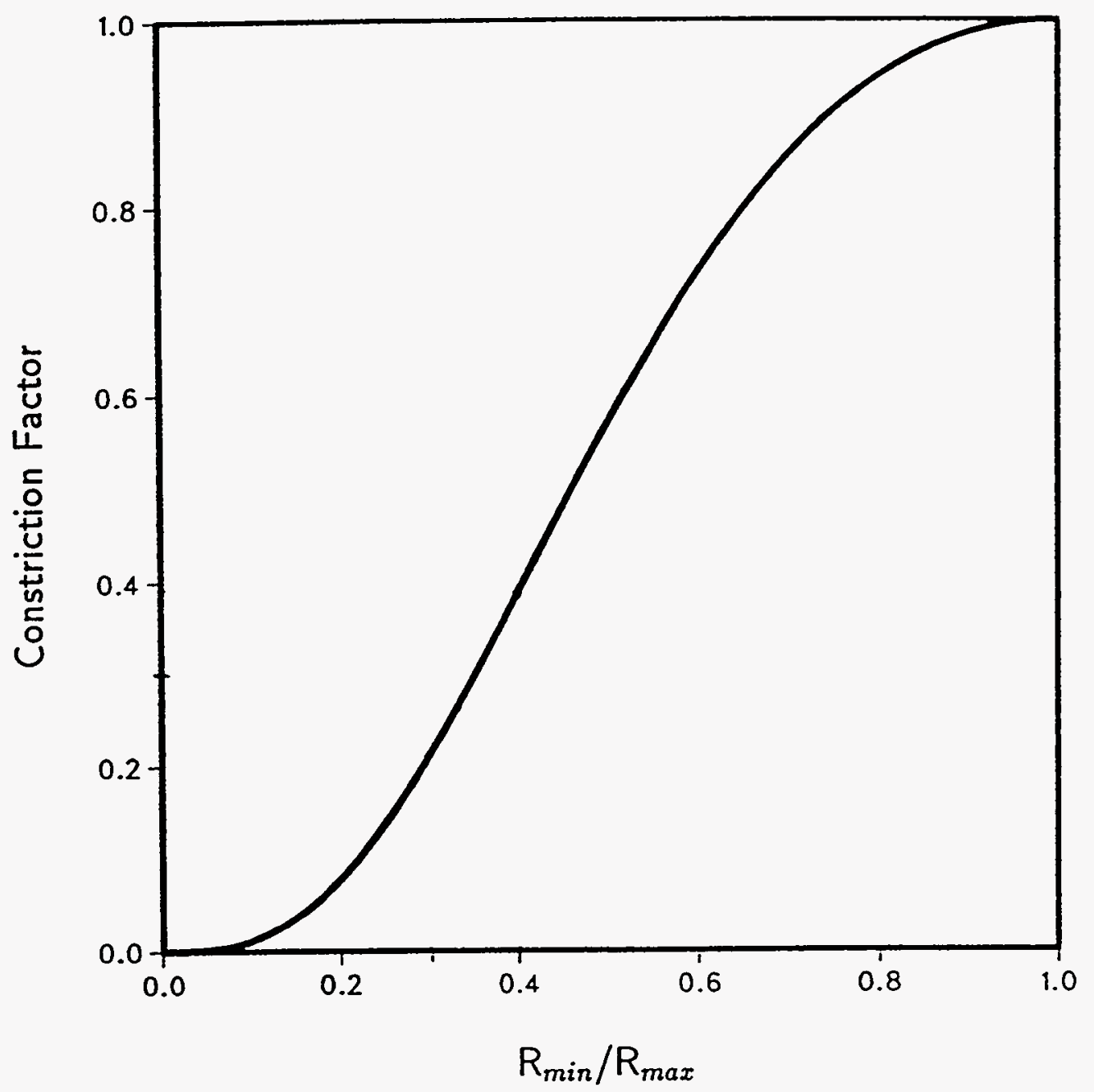

FIG. 3.4. Constriction factor for hydraulic flux, as function of the ratio of the minimum pore radius to the maximum pore radius of an individual pore. The calculated conductances of the pores must be multiplied by this factor, which account for the converging-diverging nature of the pore tubes. 


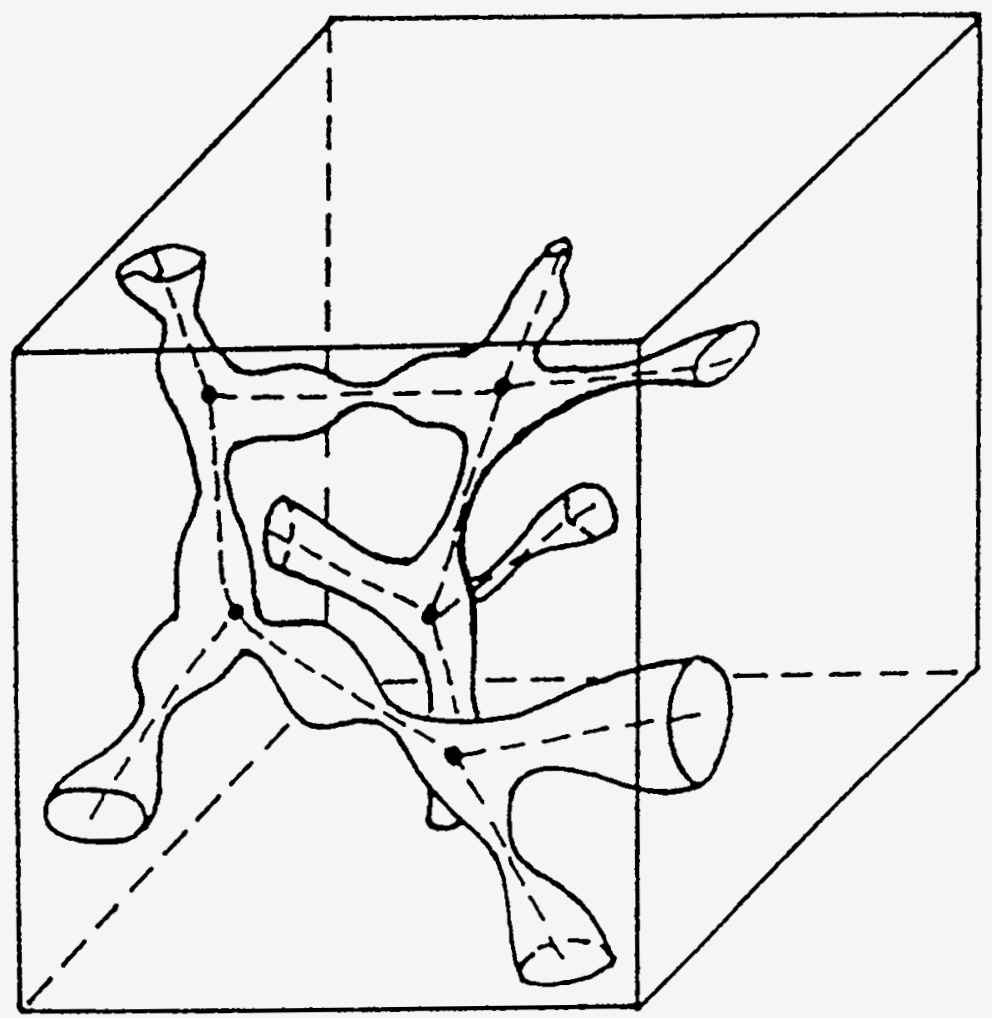

FIG. 3.5. Schematic diagram of the 'skeleton' of a microscopically inhomogeneous pore system (after Doyen, 1988). 
TABLE 3.1. Intrinsic permeability of four sedimentary rocks.

\begin{tabular}{ccc}
\hline \hline Rock & Measured & Predicted $\left(z^{\mathrm{a}}=6\right)$ \\
\hline Berea & $460^{\mathrm{b}} \mathrm{md}$ & $840 \mathrm{md}$ \\
Boise & $1.30^{\mathrm{b}} \mathrm{d}$ & $1.69 \mathrm{~d}$ \\
Massilon & $2.50^{\mathrm{c}} \mathrm{d}$ & $3.88 \mathrm{~d}$ \\
Saint-Gilles & $170^{\mathrm{d}} \mathrm{md}$ & $460 \mathrm{md}$ \\
\hline \hline
\end{tabular}

${ }^{a}$ coordination number.

b distilled water used as permeant.

'Koplik et al., 1984.

${ }^{d}$ Persoff, 1990. 


\section{PERIMETER-AREA POWER-LAW RELATIONSHIP OF PORES IN SEDIMENTARY ROCKS AND IMPLICATIONS FOR PERMEABILITY}

Since the intrinsic permeability is a measure of the viscous resistance to fluid flow through the rock pores and is controlled by the geometry and topology of the pore space, it is expected to correlate with the amount of surface area of the pore system.

In this chapter, a brief discussion is given of how the perimeter-area power-law relationship of pores, along with a pore-size distribution, can be used to estimate the permeability. Consider Fig. 4.1. If the outer circle has radius $R_{o}$ and the inner circle (dashed line) has radius $R_{i}$, then the permeability $k$ of a single such rough-walled cylindrical pore must satisfy (Berryman and Blair 1987)

$$
k_{i} \leq k \leq k_{\circ}
$$

where $k_{o}=R_{o}^{4} / 8$, and $k_{i}=R_{i}^{4} / 8$. If $R_{o}=R_{i}+\delta R$, then for small $\delta R$ we have

$$
\frac{R_{i}^{2}}{8} \leq k \leq \frac{R_{i}^{2}}{8}\left(1+4 \frac{\delta R}{R_{i}}\right)
$$

In terms of hydraulic radius this can be written as

$$
\frac{1}{2}\left(\frac{A_{i}}{P_{i}}\right)^{2} \leq k \leq \frac{1}{2}\left(\frac{A_{i}}{P_{i}}\right)^{2}\left(1+4 \frac{\delta R}{R_{i}}\right)
$$

where $A_{i}$ and $P_{i}$ are the inner tube area and perimeter respectively.

If the surface is very rough (e.g., fractal), the pore perimeter may become so large that Eq. 13 is not satisfied. Nevertheless, it follows from Eqs. 12 and 13 that an effective hydraulic radius may be used such that

$$
\left(\frac{A_{i}}{P_{i}}\right) \leq \overline{\left(\frac{A}{P}\right)} \leq\left(\frac{A_{o}}{P_{o}}\right)
$$

The parameter $\overline{\left(\frac{A}{P}\right)}$ has the significance of being the hydraulic radius of a smoothed or poor representation of the true void/solid interface. For a single straight tube (Eq. 12 ), if we make an error of $1 \%$ in estimating the tube radius, the error in the estimate of $k$ is not worse than $4 \%$. Indeed, since the fluid velocity vanishes at the pore-grain interface, the permeability $k$ should not be sensitive to surface roughness. 


\section{A. Perimeter-area power-law relationship of pores}

Perimeter-area relationships of a smooth representation of pores are estimated from scanning electron micrographs of thin sections of typical reservoir-type sedimentary rocks (Fig. 4.2). The basic method involves counting size and perimeter grid (or pixel) units for every feature in a standard scanning electron micrograph of some fixed magnification. The field imaged by the micrograph must contain a large enough number of pores to assure a statistically representative sample; we have found that 30 40 pores suffice for this purpose. The analysis was carried out using both a manual and an automated image analysis procedure to verify the accuracy of the manual technique. The manual technique involved overlaying a square grid, with grid size of $2.54 \mathrm{~mm}$, and visually counting the number of grid blocks occupied by the area of each pore, as well as the number of grid blocks that the perimeter passes through. Digital images with typical image sizes of $482 \times 640$ pixels, and 8 bits per pixel to quantify the darkness level, were used for studying the accuracy of the manual technique. The image analysis program sets a threshold level of darkness to distinguish between the pore contours and mineral grains. The digitized thin section (Fig. 4.3) then shows pore space in white, and mineral grains in black. This method was used to estimate the area-perimeter statistics for group of pores in a thin-section. It is found that the perimeter-area relationship of such a representation of the true void/solid interface satisfies the perimeter-area power-law relationship

$$
A=m P^{\gamma}
$$

where $\log m$ is the intercept on the $\log A$ axis, and $\gamma$ the slope of the $\log A-\log P$ plot (Fig. 4.4). The constants $m$ and $\gamma$ appearing in Eq. 15 are found by performing a linear regression on the $\log$ perimeter-log area data. From this analysis we find slopes ranging from 1.43 to 1.49 for the four sandstones examined, while that of an Indiana limestone is found to be 1.67 (Table 4.1).

\section{B. Permeability implications}

We now show how the area-perimeter power-law relationship of a smoothed representation of the pore space of a rock can be used, in conjunction with a pore-size distribution and a classical model for permeability, to yield reasonable estimates of permeability. The Kozeny-Carman model for transport through a porous medium is based on the idealization of the pore space as consisting of a bundle of parallel tubes, the total conductance of which is merely the sum of the individual conductances. It is traditional to then divide this result by a tortuosity factor $\tau=3$, to account for the fact that in an hydraulically isotropic rock, we would expect only one-third of 
the total number of tubes to be oriented in each of the three orthogonal directions. If $n(A)$ is the number distribution function for pores of cross-sectional area $A$ in an area of rock having total cross-section of $A_{\text {total }}$, and $C(A)$ is the conductance of each pore of area $A$, then the total conductance can be expressed as

$$
C_{\text {total }}=\int_{0}^{\infty} n(A) C(A) d A
$$

In practice, of course, the distribution function $n(A)$ will vanish for all $A$ greater than some $A_{\max }$, although it is often convenient to represent $n(A)$ by a function that drops off, say, exponentially as $A \rightarrow \infty$.

If the pore tubes were all of circular cross-section, their individual conductances would be given by the exact Hagen-Poiseuille law. The Hagen-Poiseuille solution can be modified to account for irregular cross-sections by using the 'hydraulic radius' approximation, which predicts a conductance of $A^{3} / 2 P^{2}$ for a tube of cross-sectional area $A$ and perimeter $P$. Invoking the perimeter-area power-law relationship $P=$ $m^{-1 / \gamma} A^{1 / \gamma}$, the hydraulic conductance can be expressed as $C(A)=A^{3-2 / \gamma} / 2 m^{-2 / \gamma}$. Combining this with the general expression (6) for the total conductance yields

$$
C_{\text {total }}=\frac{1}{\tau} \int_{0}^{\infty} \frac{n(A) A^{3-2 / \gamma}}{2 m^{-2 / \gamma}} d A
$$

We now define a normalized distribution function $\beta(A)=n(A) A / \phi A_{\text {total }}$, where the total porosity is defined as $A_{\text {pores }} / A_{\text {total }}$. This distribution function has the property that $\int \beta(A) d A=1$. The total conductance can now be expressed as

$$
C_{\text {total }}=\frac{\phi A_{\text {total }}}{2 m^{-2 / \gamma} \tau} \int_{0}^{\infty} A^{2(1-1 / \gamma)} \beta(A) d A
$$

We have found that the area frequency distribution of the pores (Fig. 4.5) can be well approximated by a lognormal distribution:

$$
\hat{\beta}(u)=\left(2 \pi \sigma_{u}^{2}\right)^{-1 / 2} \exp \left[-\left(u-u_{o}\right)^{2} / 2 \sigma_{u}^{2}\right]
$$

where $u=\log A, u_{o}=\log A_{o}$, where $A_{o}$ is the most probable area, and $\sigma_{u}$ is the variance of $\log A$. The corresponding mean area $A_{m}$ is larger than the most probable area, and is given by

$$
A_{m}=A_{o} \exp \left[\left(\sigma_{u} \ln 10\right)^{2} / 2\right]
$$

The permeability coefficient $k$ can then be estimated as 


$$
\begin{gathered}
k=\frac{C_{\text {total }}}{A_{\text {total }}}=\frac{\phi}{2 m^{-2 / \gamma} \tau} \int_{0}^{\infty} A^{2(1-1 / \gamma)} \beta(A) d A \\
=\frac{\phi}{2 m^{-2 / \gamma} \tau} A_{m}^{4(1-1 / \gamma)^{2}} A_{o}^{2(1-1 / \gamma)(2 / \gamma-1)} \\
=\frac{\phi}{2 m^{-2 / \gamma} \tau} A_{m}^{2(1-1 / \gamma)} \exp ^{-2(1-1 / \gamma)(2 / \gamma-1)\left[\left(\sigma_{u} \ln 10\right)^{2} / 2\right]}
\end{gathered}
$$

As with the standard Kozeny-Carman model, if the pore sizes are held constant, the predicted permeability scales with the porosity, which is to say it is proportional to the number of pores.

It is worth noting that if the perimeter-area relationship of the pores follows the law derived by Mandelbrot (1988) for islands whose boundaries are fractal: $P=\epsilon^{D} A^{D / 2}$, where $\epsilon$ is some constant that depends on the length of the measuring grid size, and $D$ is the fractal dimension of the pore perimeter, then the parameter $\epsilon$ would quantify the perimeter of the pore cross-sections when the pores are projected back into threedimensions. The parameter $\epsilon$ would in some sense represent the pore actual surface area; $k$ becomming a decreasing function of $\epsilon$. Therefore, if the rock pore boundaries are very rough (e.g., fractal), not only physical bounds for permeability would be utterly violated (Eqs. 12 and 13), but also predicted permeabilities given by Eq. 23 would be artificially lowered by several orders of magnitude.

\section{Correction for pore orientation and constrictivity}

In the two-dimensional sections under consideration, however, the pore cross- sections are randomly oriented with respect to the channel axes. The orientation effect has been taken into account by means of geometrical and stereological considerations which indicates a stereological factor of 0.40 (see Schlueter et al., 1991 for details). In addition, constrictions within the individual branch channels, i.e., pore necks and bulges, have been taken into account using an analysis based on a sinusoidal variation of cross-section which gives an hydraulic constriction factor of 0.55 .

\section{Results and discussion}

Image analysis of the pore system of Massilon sandstone yields values $A_{m}=13.5 \times 10^{4}$ $(\mu \mathrm{m})^{2}, \quad \sigma_{u}=0.43, \quad \gamma=1.43, \quad m=1.17(\mu \mathrm{m})^{2-\gamma}$. Similarly, for Berea sandstone $A_{m}=77.9 \times 10^{2}(\mu \mathrm{m})^{2}, \sigma_{u}=0.42, \gamma=1.49, m=0.66(\mu \mathrm{m})^{2-\gamma}$. Equation 23 then predicts a permeability of $10.9 \mathrm{~d}$ for Massilon sandstone, and of $1517 \mathrm{md}$ for Berea sandstone, respectively (after applying the hydraulic stereological and constriction corrections). 
The predicted values are of the same order of magnitude of the experimentally measured values of $2.5 \mathrm{~d}$ for Massilon sandstone (Koplik et al., 1984), and of $460 \mathrm{md}$ for Berea sandstone (using distilled water as permeant). Since the permeability of rocks can range over many orders of magnitude, this prediction is not trivial. Of course, more accurate estimates of the permeability will require more sophisticated models than that of parallel tubes, which somehow account for factors such as the interconnectedness of the pore tube network (Chapter III); the above examples were intended to be plausible demonstrations of how direct pore microgeometry measurements such as the area-perimeter power-law information, along with a pore-size distribution, can be used to estimate the permeability.

${ }^{1}$ Berryman, J.G., and Blair, S.C. 1987. Kozeny-Carman relations and image processing methods for estimating Darcy's constant. J. Appl. Phys. 62 (6), p.2221-2228.

${ }^{2}$ Koplik, J., Lin, C., and Vermette, M. 1984. Conductivity and permeability from microgeometry. J. of Appl. Phys. 56 (11), p.3127-3131.

${ }^{3}$ Mandelbrot, B.B. 1982. The fractal geometry of nature. Freeman, San Francisco.

${ }^{4}$ Schlueter, E.M., Cook, N.G.W., Zimmerman, R.W., and Witherspoon, P.A. 1991. Predicting permeability and electrical conductivity of sedimentary rocks from microgeometry. Proc. 32nd U.S. Symposium on Rock Mechanics, Balkema, Rotterdam, p.355-364.

${ }^{5}$ Schlueter, E.M., Zimmerman, R.W., Cook, N.G.W., and Witherspoon, P.A. 1992. Perimeter-area power-law relationship of pores in sedimentary rocks and implications for permeability. Lawrence Berkeley Laboratory, Earth Sciences Division Annual Report, LBL-31500, p.46-49. Presented at the Soc. Eng. Sci. $29^{\text {th }}$ Tech. Conf., San Diego, CA, September, 1992. 


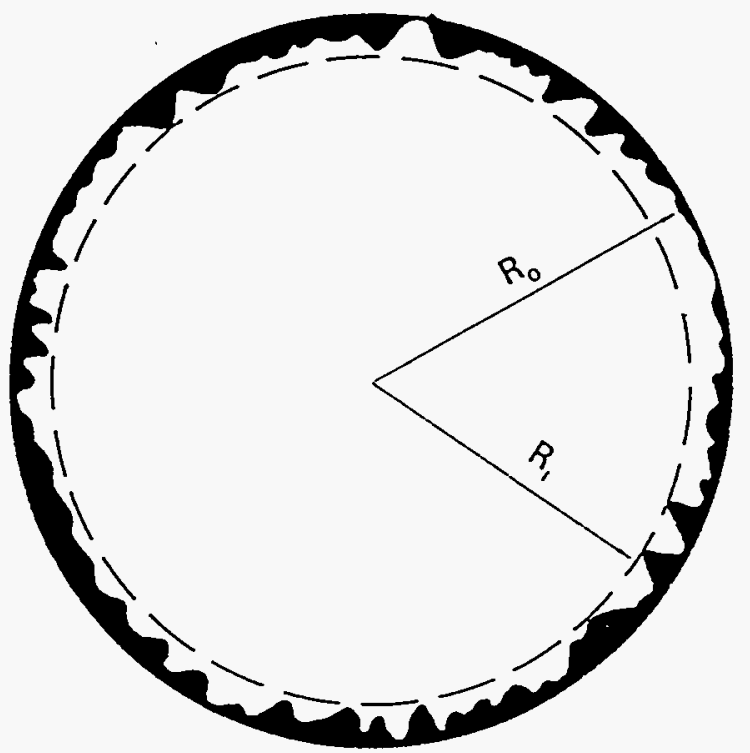

FIG. 4.1. Schematic drawing of a rough walled tube (of radius $R_{0}$ ). Surface roughness does not have a strong effect on the overall fluid permeability of a tube, because a slightly smaller tube (of radius $R_{i}$ ) is known to have a comparable permeability (after Berryman and Blair, 1987). 


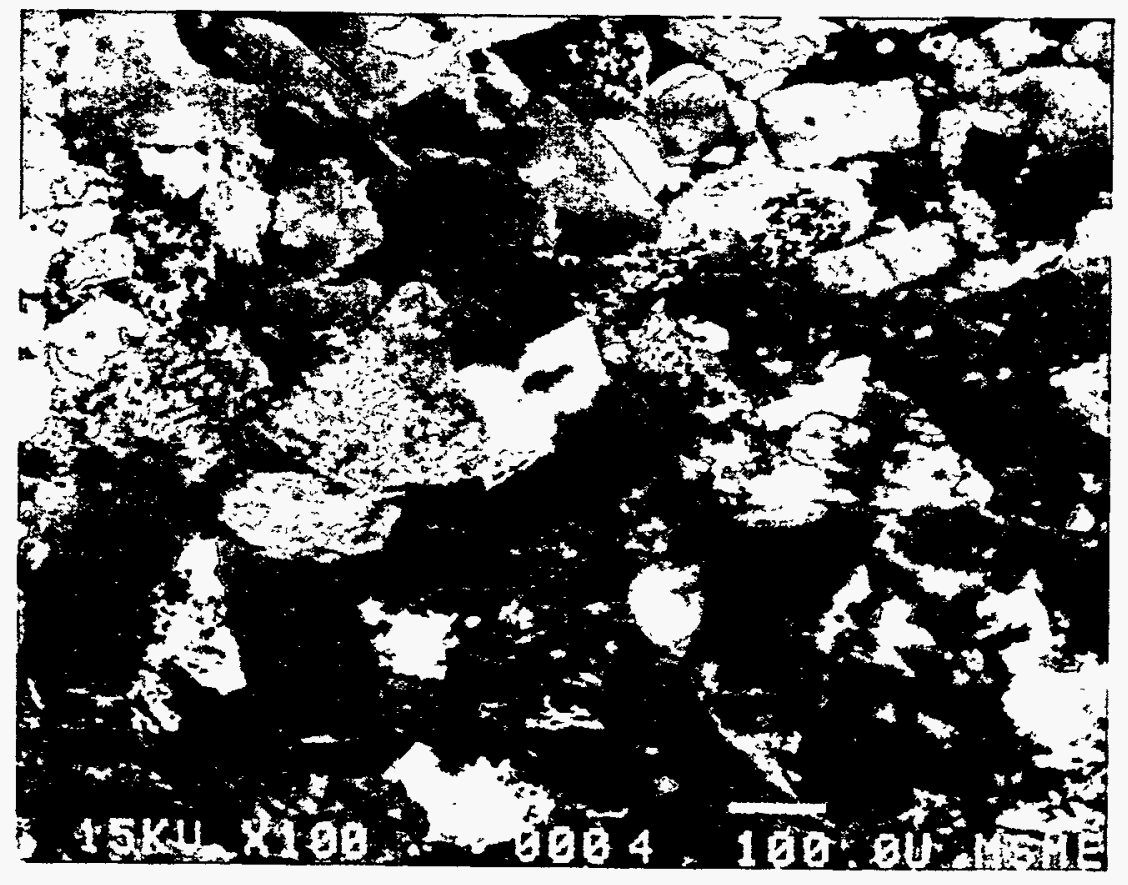

FIG. 4.2. Typical serial section of Saint-Gilles sandstone, at 100X magnification. The mineral grains of different shades are quartz, carbonate, feldspar, and muscovite, whereas the darkest regions represent pore space. 


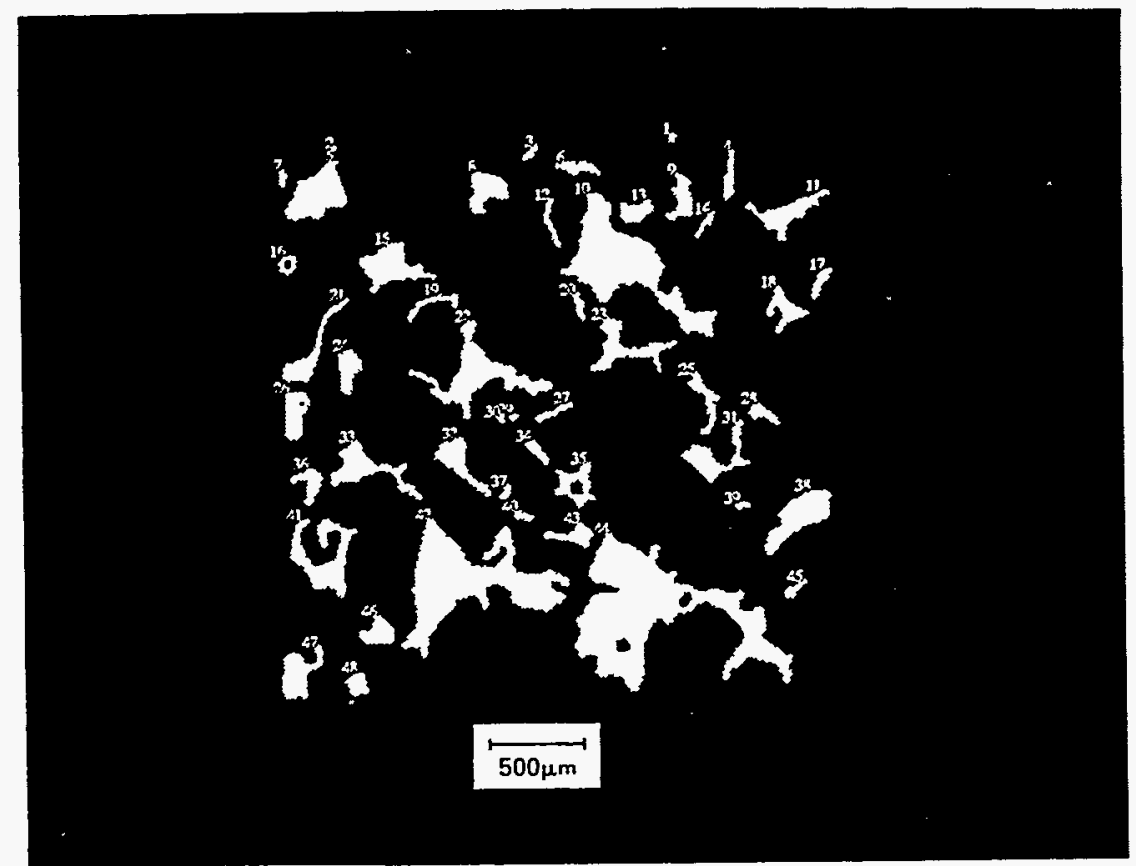

FIG. 4.3. Pore-space contours obtained from serial section of Massilon sandstone (after Koplik et al., 1984). 


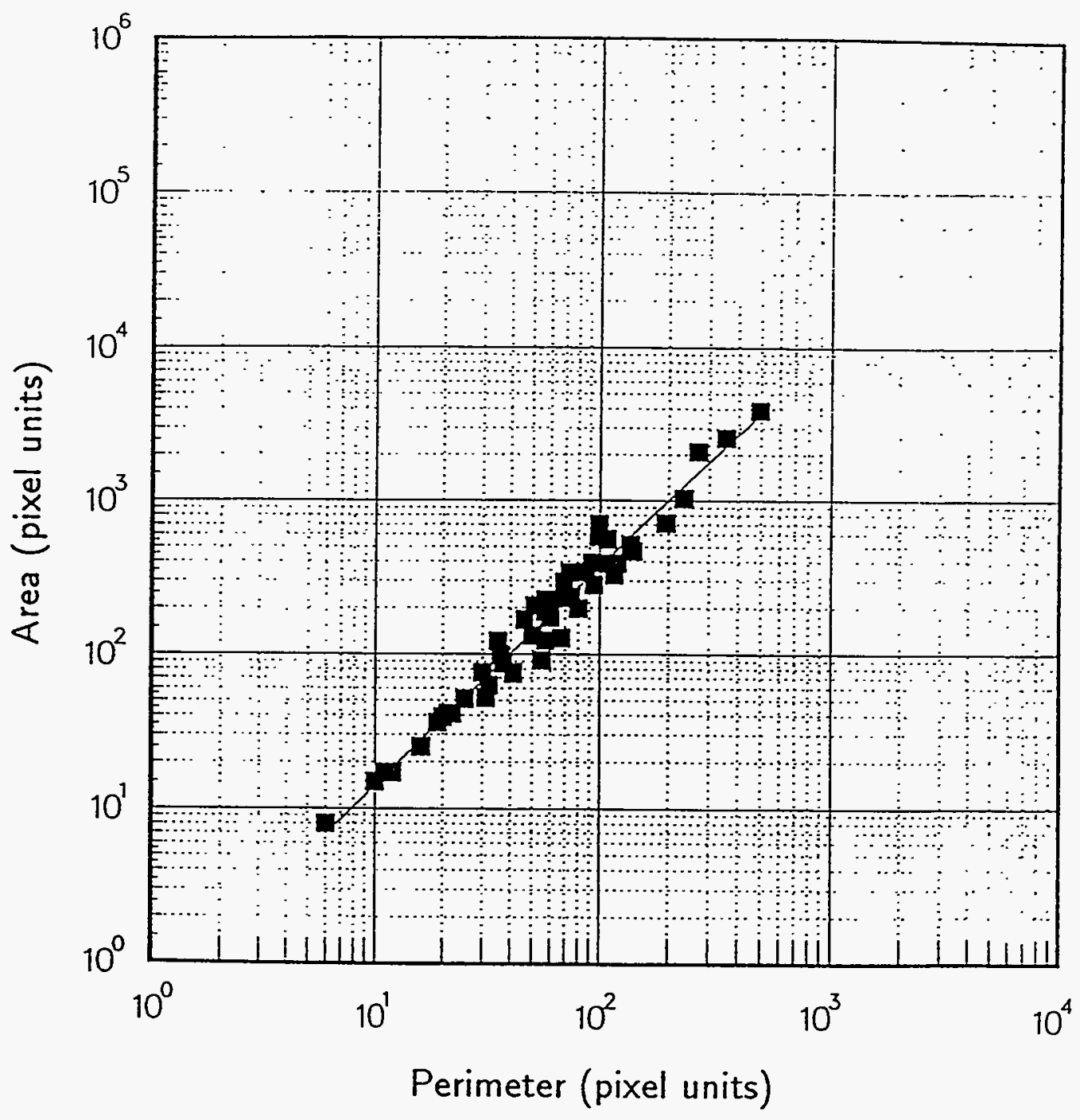

FIG. 4.4. Perimeter-area power-law relationship for Massilon sandstone. 


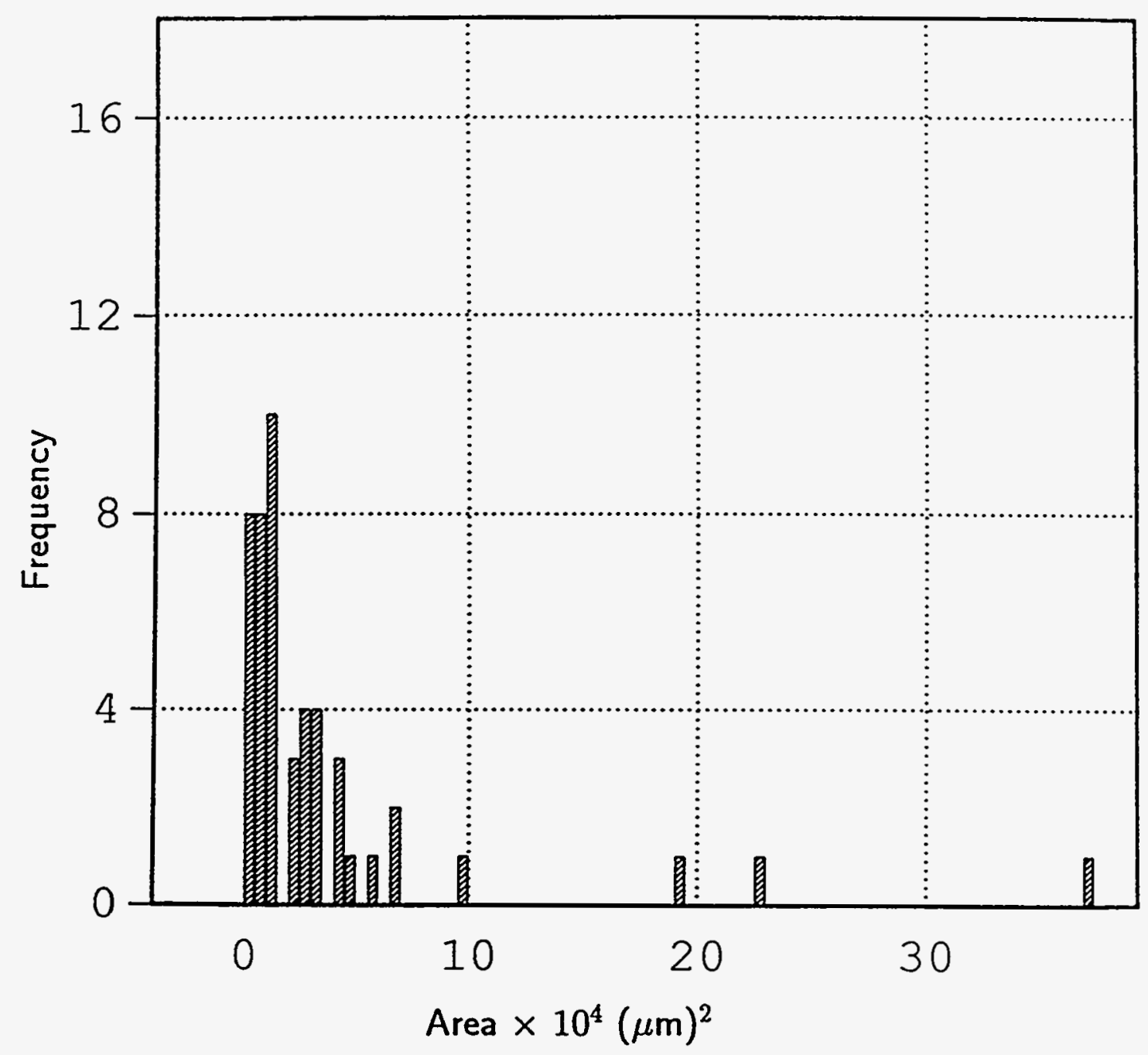

FIG. 4.5. Area frequency distribution of pores obtained from serial section of Massilon sandstone shown in Fig. 4.3. 
TABLE 4.1. Perimeter-area power-law relationship $\gamma$ parameter, and correlation coefficient $r$ measured from perimeter-area data of five sedimentary rocks.

\begin{tabular}{cccc}
\hline Rock & $\phi^{\mathrm{a}}(\%)$ & $\gamma$ & $r$ \\
\hline Berea & 22 & 1.49 & 0.99 \\
Boise & 26 & 1.43 & 0.98 \\
Massilon $^{\mathrm{b}}$ & 22 & 1.43 & 0.98 \\
Saint-Gilles $_{\text {Indiana limestone }}$ & 21 & 1.49 & 0.98 \\
\hline \hline
\end{tabular}

aporosity.

${ }^{b}$ Koplik et al. 1984. 


\section{PREDICTING THE ELECTRICAL CONDUCTIVI'IY UY SEDIMENTARY ROCKS FROM MICROGEOMETRY}

A primary objective of this research is to understand, through analysis and experiment, how pore structure affects the electrical and hydraulic properties of rocks, and to develop equations relating these macroscopic properties to the microscopic geometry and structure of the pore space.

In this study, the electrical conductivity of sedimentary rocks is predicted from the microscopic geometry of the pore space. We have preserved the same intergranular pore model structure used to predict hydraulic permeability (Chapter III). The cross-sectional areas of the individual pores are estimated from 2-D scanning electron micrographs of rock cross-sections (Figs. 5.1 and 5.2). The electrical conductivity of the individual pores is determined from the geometrical parameters, using Ohm's law. In the section under consideration, the pore cross-sections are assumed to be randomly oriented with respect to the directions of the channel axes. The orientation effect has been corrected by means of geometrical and stereological considerations. Account is also taken for possible variation of the cross-sectional area along the length of each tube, e.g., pore necks and bulges. The effective medium theory of solid state physics is then used to replace each individual conductance with an effective average conductance of each pore. Finally, the pores are assumed to be arranged on a cubic lattice which allows the calculation of overall macroscopic values for the electrical conductivity. Preliminary results, using Berea, Boise, and Saint-Gilles sandstone, show fair to good agreement between the predicted and measured electrical conductivities with essentially no arbitrary parameters in the model. In particular, good agreement was found in case of a poorly cemented rock such as Saint-Gilles sandstone, whereas the agreement was not very good for two well-cemented rocks, Boise and Berea. The possible reason(s) for this effect are under investigation.

\section{A. Effect of cross-sectional shape}

The electric conductance per unit length of each cylindrical tube of area $A$ is

$$
c_{e}=\frac{A}{\rho_{w}}
$$

where $\rho_{w}$ is the resistivity of the fluid; this expression is exact regardless of the pore shape.

\section{B. Correction for pore orientation}

In the two-dimensional sections under consideration, however, the pore cross- sections are randomly oriented with respect to the directions of the channel axes. The 
orientation effect has been corrected by means of the following gevinemllar aru sureological considerations (cf., Underwood, 1970),

$$
A_{\text {actual }}=\left\langle\frac{1}{\cos \theta}\right\rangle^{-1} A_{\text {measured }}
$$

where the brackets denote a spherical average for pores of random orientation, i.e.,

$$
\left\langle\frac{1}{\cos \theta}\right\rangle=\frac{\int_{0}^{\pi} \int_{0}^{\theta_{\max }}(\cos \theta)^{-1} \sin \theta d \theta d \phi}{\int_{0}^{\pi} \int_{0}^{\theta_{\max }} \sin \theta d \theta d \phi}
$$

with $\theta_{\max }=\arctan (L / D)$, and $L / D$ being the maximum ratio of pore length to diameter. Using an average value of $L / D=5$, as estimated from the micrographs, we find that

$$
A_{\text {actual }}=0.61 A_{\text {measured }}
$$

(Evaluation of the integral appearing in equation 26 shows that the factor 0.61 is relatively insensitive to the value chosen for $L / D$ ).

\section{Correction for constrictivity}

Constrictions within the individual branch channels, i.e., pore necks and bulges, have been taken into account using an analysis based on a sinusoidal variation of cross-section. In the electric conductance analysis, for example, the factor accounts for the ratio of $\left\langle R^{-2}\right\rangle^{-1}$, which governs the conductance of a tube of varying radius, to $\langle R\rangle^{2}$, which is the value estimated from the micrographs. We have estimated a throat-to-pore radius ratio of 0.5 from a pore cast of Berea sandstone, and tentatively use this value for each rock. This ratio then indicates an electric constriction factor of 0.86 (Fig. 5.3).

\section{Effective medium theory}

Study of scanning electron micrographs of Berea sandstone has indicated the presence of a statistically isotropic 3-D pore structure. These observations have led to the assumption that the pores of varying size are arranged on a cubic lattice, so that the coordination number of the network is 6 . The effective medium theory from solid state physics is then used to replace each individual conductance with an effective average conductance as follows. For a general discrete distribution of conductances, the resulting equation defining the effective conductance is (Kirkpatrick, 1973) 


$$
\sum_{i=1}^{N} \frac{c_{e f f}-c_{i}}{[z / 2-1] c_{e f f}+c_{i}}=0
$$

where the sum is over all $N$ conductive elements, and $z$ is the averarage coordination number of the lattice. For a coordination number of $z=6, \mathrm{Eq} .28$ has been solved numerically to allow for the calculation of the effective medium conductance, given the individual conductances.

The electrical conductivity is often quantified by the 'formation factor', which is the ratio of the resistance of the brine-saturated rock to the resistance of an equal volume of brine. Thus, a unit cubic cell is used to relate the effective electric tube conductance to the continuum values of electrical conductivity by means of the following expression,

$$
F=\frac{\tau A_{\text {photo }}}{N A_{\text {eff }}}
$$

where $A_{e f f}$ is the effective pore area of the individual tubes, $N$ the number of pore tubes in the micrograph, $A_{p h}$ the area of the photograph, and $\tau$ the tortuosity of a cubic lattice, which is 3 .

\section{E. Results and discussion}

Preliminary results are presented in Table 5.1. Fair to good agreement was found between measured and predicted electrical conductivities, for a variety of sandstones when using the cubic lattice model, with essentially no arbitrary adjustable parameters. In particular, good agreement was found in case of a poorly cemented rock such as Saint-Gilles sandstone, whereas the agreement was not very good for two well-cemented rocks, Berea and Boise. The possible reasons(s) for this effect will be investigated experimentally in the next chapter (Chapter VI). In this study, we have preserved the intergranular pore model structure used to predict intrinsic permeability from microgeometry. However, it is likely that in some cases this basic pore model structure needs to be modified to incorporate relevant rock geometrical and topological characteristics important for electrical conductivity.

${ }^{1}$ Kirkpatrick, S. 1973. Percolation and conduction. Rev. Mod. Phys. 45 (4), p.574587. 
${ }^{2}$ Scheidegger, A.E. 1974. The physics of flow through porous media. University of Toronto Press.

${ }^{3}$ Schlueter, E.M., Cook, N.G.W., Zimmerman, R.W., and Witherspoon, P.A. 1991. Predicting permeability and electrical conductivity of sedimentary rocks from microgeometry. Proc. 32nd U.S. Symposium on Rock Mechanics, Balkema, Rotterdam, p.355-364.

${ }^{4}$ Underwood, E.E. 1970. Quantitative stereology. Reading, Mass. Addison-Wesley. 


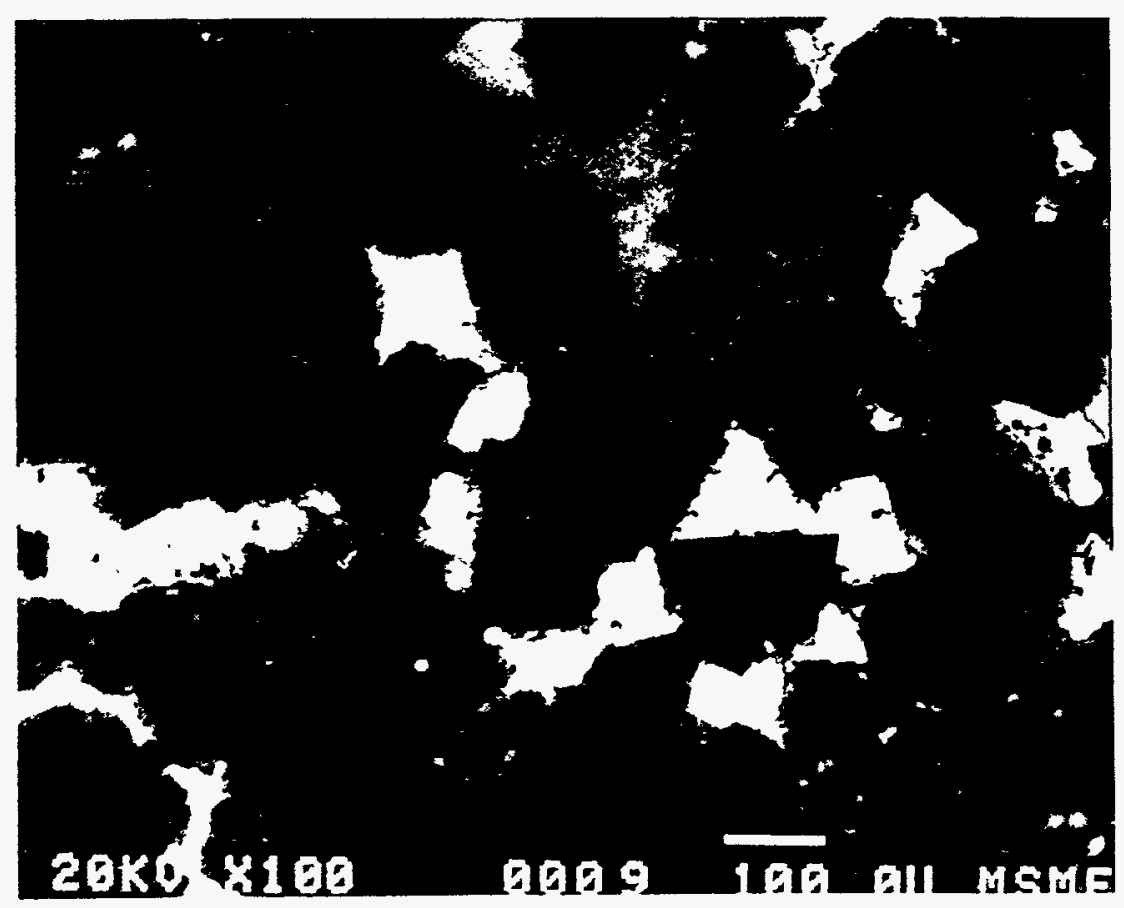

FIG. 5.1. Typical scanning electron microscope photomicrograph of a Berea sandstone section. The rock is composed mainly of quartz grains (dark gray), feldspar grains (medium gray), and products of grain dissolution (light gray). The pore space is impregnated with Wood's metal alloy (white), and epoxy (black). Actual width of field is about $1 \mathrm{~mm}$. 


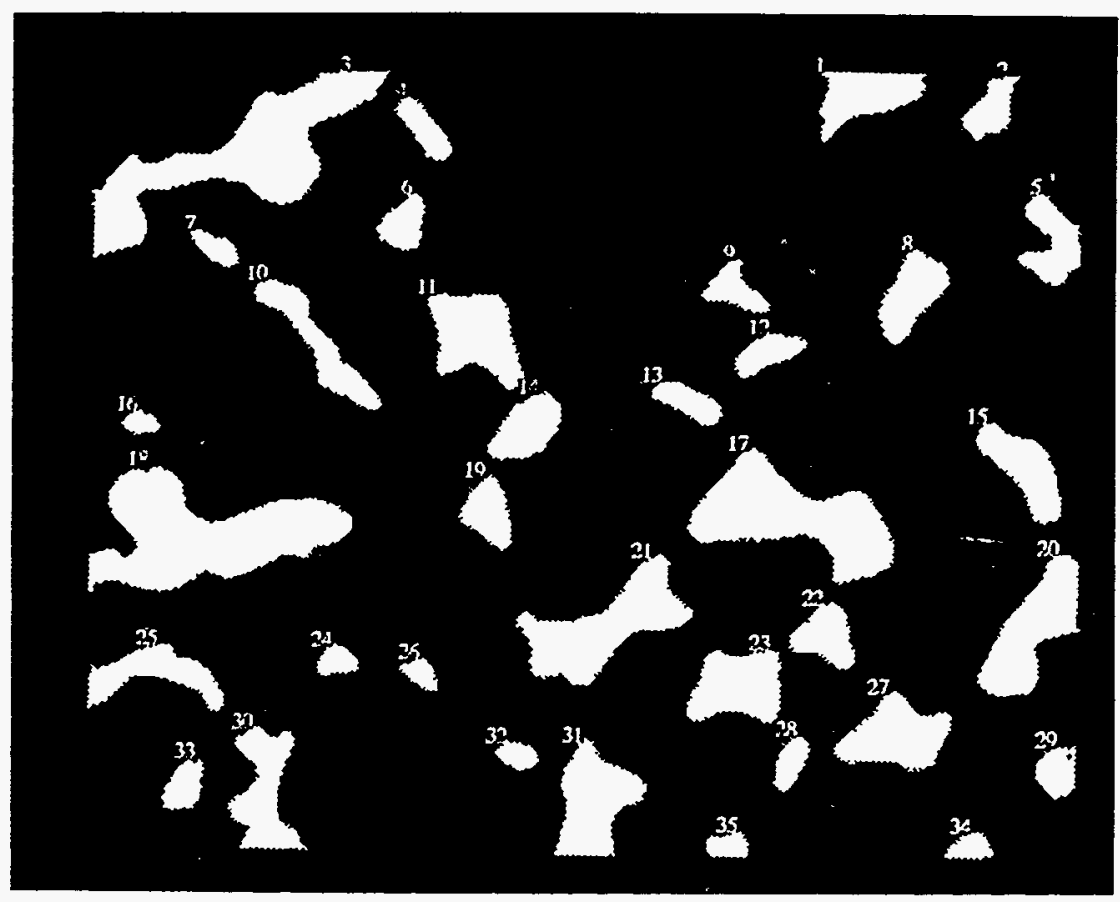

FIG. 5.2. Pore-space contours obtained from computerized image analysis of the section shown in Fig. 5.1. 


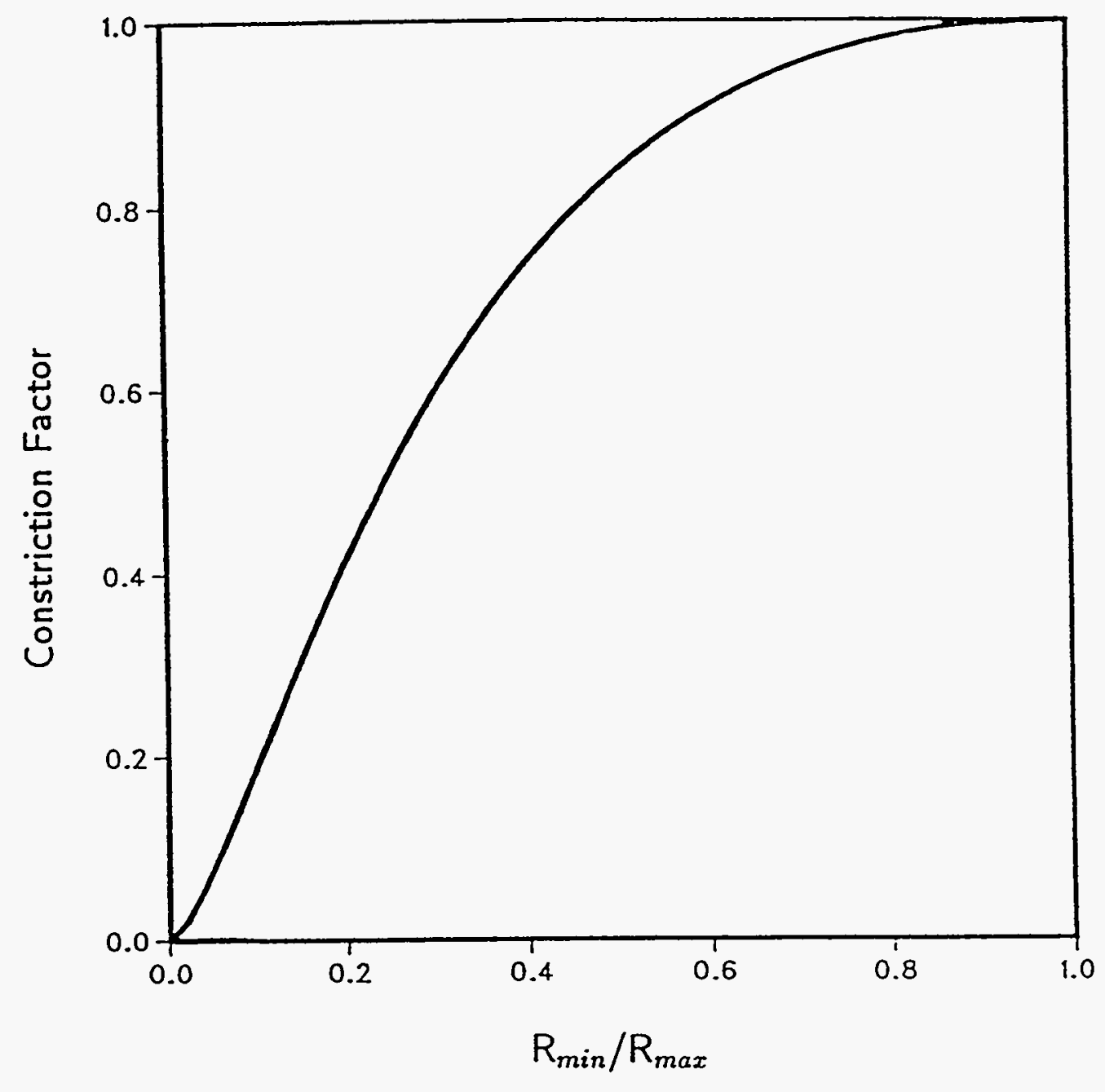

FIG. 5.3. Constriction factor for electric flux, as functions of the ratio of the minimum pore radius to the maximum pore radius of an individual pore. The calculated conductances of the pores must be multiplied by these factors, which account for the converging-diverging nature of the pore tubes. 
TABLE 5.1. Formation factor of three sedimentary rocks.

\begin{tabular}{ccc}
\hline \hline Rock & Measured $^{\mathrm{a}}$ & ${\text { Predicted }\left(z^{\mathrm{b}}=6\right)}^{\mathrm{b}}$ \\
\hline Berea & 15.9 & 44.1 \\
Boise & 11.0 & 27.2 \\
Saint-Gilles & 13.5 & 13.1 \\
\hline
\end{tabular}

aninc nitrate solution used as electrolyte.

${ }^{b}$ coordination number. 


\section{FORMATION FACTOR AND THE MICROSCOPIC DISTRIBUTION OF WETTING PHASE IN PORE SPACE OF BEREA SANDSTONE}

The transport properties of porous rocks such as hydraulic and electrical conductivity are determined by pore topology, geometry, and the physics and chemistry of mineral-fluid and fluid-fluid interactions. A primary focus of our research is to understand with analysis and experiment how these factors affect the property under consideration. On our electrical conductivity experimental studies, we have used a wetting fluid that can be frozen in place: (1) to allow measurement of effective properties to an electrolyte solution of the rock pore spaces not occupied by a wetting fluid, (2) to allow direct observation and analysis of the wetting fluid distributions at each fluid saturation regime, and (3) to understand how the wetting fluid invasion process is controlled by rock pore structure and topology with the aid of a complete pore cast. In addition, disseminated clay, often in the form of aggregates, may line the rock pores or be distributed between the grains of the matrix. The clay minerals act as a separate conducting path additional to that contributed by the saline solution in the rock pores. The conductivity of the rock containing disseminated clay therefore has two components: one is the conductivity of the fluid-filled pores of the rock; the other one is the conductivity of the disseminated clays which are the solid phases that primarily exhibit surface reactivity in rocks and soils (Sposito, 1984). This is a direct consequence of the fact that clay particles have large surface areas. In order to measure surface conductance, the geometry of the system must be known. In the case of rocks, the exact pore geometry that affects the total conductance is unknown. Furthermore, it can change due to dispersion and flocculation of the clay minerals in the pores. Thus it is forseen that the presence of clay may greatly complicate the quantitative evaluation and interpretation of experimental data. In our experimental studies, we have isolated the effect of clay on formation factor and assesed the surface conductance contribution due to the presence of clays.

The conductivity of a rock (consisting of electrically nonconductive particles) saturated with a conducting electrolyte has been shown by Archie (1942) to depend upon the conductivity of the electrolyte and the geometrical (e.g., formation) factor. In such a system, the electrical conductance of the saturated rock is proportional to the conductance of the electrolyte solution; the constant of proportionality (always $>1$ ) is a geometrical factor called the formation factor. The formation factor of a fully saturated rock is defined as

$$
F=\frac{\rho(\text { electrolyte saturated sample })}{\rho(\text { electrolyte solution })}
$$

where $F$ is the formation factor, and $\rho$ the resistivity. 
Thè assumptions implicit in the formation factor concept are: (1) The conduction proces̀s is electrolytic and it occurs only through the network of saline solution filling the pores, (2) the properties of the saline solution filling the pores are uniform, and (3) there are no surface conductivity effects. In such a system, the plot of the electrical conductance of the saturating solution versus the electrical conductance of the rock saturated with the solution is a straight line passing through the origin.

When more than one fluid is present, e.g., oil/water, the empirically determined Archie saturation is often found to vary according to (Archie, 1942; King et al., 1988)

$$
F=\phi^{-m} S_{w}^{-n}
$$

where $F$ is the effective formation factor, $\phi$ is the porosity, $S_{w}$ the electrolyte saturation, and $m$ and $n$ dimensionless empirically-determined constants. The value of $m$ varies with the extent to which a rock is cemented, from about 1.3 to 2.3 (Wyllie, 1963). The value of $n$ depends to a large extent on the wettability of a reservoir rock (Anderson, 1986). In rocks that are wholly oil-wet, i.e., rocks in which the water is the nonwetting phase, $n$ ranges from 3 to about 4 . If the rock surface is entirely water-wet, the value of $n$ lies between 1.8 and 2.0 (Wyllie, 1963). The main assumptions implicit in the Archie saturation equation are: (1) the saturation/resistivity relation is unique (i.e., only one resistivity is measured at a given saturation), (2) $n$ is constant for a given porous medium, and (3) all the saline solution contributes to the flow of electric current. To test these assumptions, we have analyzed the electrical conductivity experimental data in light of the role of the pore structure and topology in the wetting fluid invasion process with the aid of the fluid distributions at each saturation regime and a complete pore cast.

Laboratory electrical conductivity data for fully and partially-saturated samples of Berea sandstone with a wetting fluid is presented. In our study, we employ a wetting fluid (paraffin wax) that can be frozen in place at controlled saturations to allow us to examine the occupied pore space after the experiment. The effective formation factors for an electrolyte in the pore spaces not occupied by the paraffin are measured at various saturations after solidifying the paraffin in place. The effect of clay and other surface reactive minerals on Berea sandstone formation factor is first isolated and their surface conductance contribution to overall conductivity assessed. The electrical conductivity experimental data is analyzed in light of the role of the pore structure on the wetting fluid invasion process with the aid of direct observation of fluid distributions at each saturation regime, a complete pore cast, and its corresponding rock section. Finally, the effect of partial hydrocarbon saturation on overall Berea sandstone conductivity, and on the Archie saturation exponent $n$ is studied. 


\section{A. Apparatus and procedure}

The apparatus that was used in this study was designed to simultaneously measure both hydraulic and electrical conductivity (Fig. 6.1) (Schlueter et al., 1992). The rock core of $50 \mathrm{~mm}$ in diameter and $50 \mathrm{~mm}$ in length was encased in its rubber jacket and placed in the test cell. The test cell base was connected directly to the bottom of the sample and had a centrally located orifice to allow fluids to flow through the mounted core. The electrodes were connected to the top and bottom of the sample so that any current flow between them necessarily passed through the core. A confining pressure of $50 \mathrm{psi}(3.4 \mathrm{~atm})$ was applied via nitrogen gas. Fluid flow through the core was controlled by a syringe pump providing a constant flow rate of $200 \mathrm{ml} / \mathrm{hr}$. The basic procedure used for measuring the formation factor in a $100 \%$ electrolyte saturated sample was to first fully vacuum saturate each core with distilled water. An aqueous zinc nitrate solution of known resistivity was then flowed through the core and continued for sufficient time to establish constant pressure and resistance readings. It was found that about four pore volumes of electrolyte were needed to achieve steady state. In our experiments, we used samples of Berea sandstone, which is a homogeneous sedimentary rock used as a reference rock in the petroleum industry. The intrinsic permeability and porosity of a Berea sandstone core are about $600 \mathrm{md}$ $\left(600 \times 10^{-15} \mathrm{~m}^{2}\right)$ and $22 \%$, respectively. It is estimated to be of Missisipian age and is found in Berea, Ohio.

\section{B. Effect of partial hydrocarbon saturation}

The conductivity of the pore system can be varied either by altering the salinity of the water in the pores or by changing the quantity of water of a particular salinity by introducing another solid/fluid phase into the pore space. To determine the effect of partial fluid saturation on formation factor, we utilized Berea sandstone samples that had been permeated with a sequence of triple distilled water (to measure the hydraulic conductivity), followed by a $0.5 \mathrm{M}$ zinc nitrate solution and again flushed with distilled water. This procedure was applied to every sample to find the formation factor before the paraffin application (Table 6.1). The samples were then oven dried. After measuring the formation factor, the samples were partially filled with paraffin wax at controlled saturations of $20 \%, 40 \%, 50 \%, 60 \%$, and $69 \%$. The hydrocarbon paraffin is a wetting phase with a density of $0.76 \mathrm{~g} / \mathrm{cm}^{3}$, and a melting point of $56^{\circ} \mathrm{C}$. It is applied at temperatures higher than its melting point in the core axial direction, and until uniform saturation is achieved throughout the sample (Fig. 6.2). The paraffin is then solidified in place at ambient temperature conditions. After the hydrocarbon paraffin application, the rock grain surfaces became hydrophobic. To 
measure the effective formation factor, the rock samples that were partially saturated with paraffin were permeated with a $0.5 \mathrm{M}$ zinc nitrate solution at $\mathrm{pH}$ of about 4 . The effective formation factor vs. paraffin saturation is presented in Fig. 6.3 (Case A). Experiments in which the formation factor had not been measured previous to paraffin impregnation were repeated on a new and clean set of samples. The result is shown in Fig. 6.3 (Case B). In summary, good agreement within experimental error was found between the two sets of experiments $A$ and $B$.

\section{Effect of clay}

Berea sandstone rock contains about $10 \%$ clay minerals coating the available pore space. X-ray diffraction studies by Khilar and Fogler (1984), in conjunction with scanning electron microscopy (Fig. 6.4) and energy dispersive x-ray analysis (Fig. 6.5 ), indicate that Berea sandstone contains about $8 \%$ by weight of dispersable and swelling clays (mainly kaolinite with some illite, and smectite), $80 \%$ quartz, and $12 \%$ feldspar. To reduce the tendency for the clays to disperse, a bivalent cation such as zinc was used. For solutions with cations of valence $>1$, at $\mathrm{pH} 7$, no critical salt concentration below which clay is released from Berea sandstone pore walls has been found (Khilar and Fogler, 1984). It is important to recognize that the clay minerals present in the rock have been immobilized by the application of the hydrocarbon paraffin coating the pores. Therefore, the formation factor extrapolated to electrolyte saturation of unity (i.e., no hydrocarbon paraffin) corresponds to the formation factor of the clean rock without clays and without any surface conduction effects caused by surface reactive minerals that may be present in the rock. The formation factor at paraffin saturation of zero, $F=18$ (Fig. 6.3), has been extrapolated from a plot of the multiplicative inverse of the residual formation factor vs. paraffin saturation. This hypothesis was verified by partially removing clays in a Berea sandstone core with an acid treatment of a mixture of $6 \%$ hydrochloric and $1.5 \%$ hydrofluoric acids (Suarez, 1991). After the core was treated and clays flushed out, the formation factor was found to be 16.4 , larger than the average formation factor of 15.7 that was measured for samples containing clay (Table 6.1).

\section{Effect of surface conductance}

To investigate the magnitude of surface conductance contribution due to clays and other surface reactive minerals on formation factor, we investigated the influence of solution concentration on rock electrical conductivity. For that purpose, resistivity experiments were performed on two Berea sandstone cores. The first core was permeated with solutions of zinc nitrate at increasing concentrations of $0.05 \mathrm{M}, 0.1 \mathrm{M}$, 
and $0.5 \mathrm{M}$ while measurements of resistivity were taken. After equilibrium had been reached with one solution, another zinc nitrate solution of less resistivity was flowed through the core, and a constant resistance reading was again obtained. To obtain a more complete set of electrical conductivities measurements at low electrolyte concentrations, the procedure was repeated on a new core with solutions that included salinities $0.005 \mathrm{M}$ and $0.01 \mathrm{M}$ zinc nitrate ( $\mathrm{pH}$ of the effluent was found to vary from about 4.5 to about 4.0, respectively). The data thus obtained enabled the formation factor of the rock to be computed, and also confirmed the ability of an invading fluid to displace interstitial water from a rock core. The results are given in Table 6.2. The conductivity of the fluid $\left(\kappa_{w}\right)$ vs. the conductivity of the fully saturated rock $\left(\kappa_{r}\right)$ data are plotted in Fig. 6.6. As observed, minor deviations from the Archie formation factor concept are present at low electrolyte concentration. There is a rapid initial increase in rock conductivity as the solution concentration increased up to about $0.005 \mathrm{M}$. When the solution in equilibrium was more concentrated than $0.005 \mathrm{M}$, there was a decrease in rock conductivity with an increase in solution conductivity until a solution concentration of about $0.05 \mathrm{M}$. Thereafter, the formation factor is constant at least over the range of concentrations studied. The shape of the $\left(\kappa_{w}-\kappa_{r}\right)$ curve at low electrolyte concentrations may be interpreted in light of the mechanisms and factors controlling clay-fluid interactions as they relate to clay-water electrolytic conduction. Berea sandstone contains mainly kaolinite clay. At $4.0<$ $\mathrm{pH}<4.5$, and electrolyte concentrations lower than $0.01 \mathrm{M}$, experiments in kaolinite clay by Williams and Williams (1978) (using sodium cloride as electrolyte) (Fig. 6.7), show that an increase in solution concentration produces a decrease in ion mobility, and thus a reduction in electrical conductivity. In addition, for clay-water systems, an increase in salt concentration is expected to compress the diffuse double layer, to decrease the electrical interaction between the cations and the clay surface, and to reduce the electrical conductivity. At higher electrolyte concentrations the double layer compresses further, there is a more energetic conductive path through the solution, and the $\left(\kappa_{w}-\kappa_{r}\right)$ plot becomes linear.

The trend of change in rock electrical conductivity at low electrolyte concentrations does reflect the contribution to surface conduction of clays and other surface reactive minerals. However, our experimental results show that for Berea sandstone, the surface conduction component represents a minor contribution to overall electrical conductivity, and therefore can be ignored for most applications.

\section{E. Effect of pore structure and topology}

To understand how pore structure and topology control the transport property of consideration, the electrical conductivity data (Fig. 6.3) have been studied in light 
of the wetting fluid distributions at each saturation regime (Figs. 6.8, 6.9, and 6.10) with the aid of a complete rock pore cast (Fig. 6.11) and its associated rock section (Fig. 6.12). The rock pore cast was obtained from a rock specimen which had been fully impregnated with Wood's metal alloy and the quartz grains removed by hydrofluoric acid. The rock pore cast and its associated rock section clearly reveal that the pore space is composed of grain contact porosity (e.g., thin sheets and micropores), and intergranular porosity. Figure 6.8 shows a scanning electron microscope micrograph collage of a Berea sandstone specimen that has been partially saturated with approximately $20 \%-30 \%$ paraffin. The gray phase corresponds to quartz grains, the white phase corresponds to pores that have been impregnated with paraffin, and the black phase corresponds to the remaining pore space filled with blue epoxy for imaging purposes. The paraffin has invaded grain contact pore space (i.e., thin sheets and micropores) and intergranular pore space connected by smaller throats while only coating available intergranular channels connected by larger throats. A substantial effect on effective formation factor is observed. Therefore, the fraction of the pore structure connected by smaller constrictions (e.g., grain contact pore space) provides important alternative routes to intergranular conduits connected by larger throats for the ions to travel. Figure 6.9, displays a scanning electron microscope collage of a rock specimen partially saturated with approximately $40 \%-50 \%$ paraffin. At this stage, we are filling intergranular conduits connected by the larger throats. Also, a portion of the electrolyte has apparently lost continuity as the paraffin saturation is increased over $\sim 30 \%$ so that the resistivity increased at a faster rate. Hence, a larger effect on effective formation factor is observed. Figure 6.10, displays a scanning electron microscope collage of a rock specimen partially saturated with approximately $60 \%-70 \%$ paraffin. We have filled almost all intergranular conduits connected by larger throats. A few intergranular pores not well connected still remain unfilled. When paraffin saturation is about $70 \%$ the whole pore structure behaves as disconnected.

\section{F. The Archie saturation exponent}

Electrical conductivity data presented in Fig. 6.3 (Case B) have been replotted on a logarithmic scale for the effective formation factor vs. electrolyte saturation (Fig. 6.13). To understand their physical significance, the plot has been divided in three zones: zone I, a linear zone between saturations of the electrolyte of about $0.7\left(S_{\text {crit }}\right)$ and 1, with an Archie exponent of $\mathrm{n} \sim 3$; zone II, a linear zone between electrolyte saturations of about 0.5 and 0.7 , with an Archie exponent of $n \sim 5$; and zone III, a zone for electrolyte saturations less than 0.5. Zone I, with Archie saturation exponent of $\sim 3$, reflects the fact that grain contact pore space (i.e., thin sheets and micropores), and intergranular pore space connected by the smaller throats provide 
important alternate paths to intergranular conduits connected by larger throats for the ions to travel. Zone II, with Archie saturation exponent of $\sim 5$, reflects the fact that as the electrolyte saturation is lowered below $S_{\text {crit }}$ part of the rock structure composed of pores connected by the smaller throats becomes inactive. In addition, intergranular conduits connected by larger throats start being filled and partially filled with paraffin. Thus, part of the electrolyte available for the transport of ions loses continuity and the resistivity is increased at a faster rate.

\section{G. Summary}

The formation factor of a partially saturated rock with a wetting phase is controlled by the rock structure and topology as well as the physics and chemistry of mineralfluid interactions. To understand the relationships, we have measured the effective formation factor to an electrolyte of the pore spaces not occupied by a wetting fluid (paraffin), after solidifying the fluid in place. It is important to recognize that when the rock is partially saturated with the hydrocarbon paraffin, the clay minerals present in the rock pore space are immobilized. Thus, the formation factor extrapolated to electrolyte saturation of unity (and paraffin saturation of zero), $F=18$, corresponds to the formation factor of the 'clean' rock (e.g., without clay). Eventhough the change in trend of rock electrical conductivity at low electrolyte concentrations does represent the contribution of surface conduction due to clays, this contribution is negligible. The effective formation factor data have been studied in light of the wetting fluid distributions observed at different saturations levels with the aid of a complete pore cast and its associated rock section. Our analysis shows that: (1) $\sim 30 \%$ of the pore space comprises grain contact pores (thin sheets and micropores), and intergranular pores connected by smaller throats, $(2) \sim 40 \%$ of the pore space comprises intergranular conduits composed of pores connected by larger throats, and (3) $\sim 30 \%$ of the intergranular pore space remains disconnected. The grain contact pore space of large surface area (thin sheets), micropores and intergranular pores connected by smaller throats provide important alternate paths to the intergranular conduits connected by larger throats for the ions to travel. Therefore, for a consolidated rock such as Berea sandstone, we find no unique relationship between effective formation factor and electrolyte saturation, and no unique definition of the Archie saturation exponent, $n$, for the full range of saturation. Finally, Archie saturation exponent $n$ is found to vary from approximately 3 when connected grain contact pore space (i.e., thin sheets and micropores), and intergranular pores connected by smaller throats are filled with hydrocarbon paraffin to approximately 5 when intergranular conduits connected by the larger throats are filled with hydrocarbon paraffin, with a critical electrolyte saturation $\left(S_{\text {crit }}\right)$ of 0.7 . 
${ }^{1}$ Anderson, W.G. 1986. Wettability literature survey-Part 3: The effects of wettability on the electrical properties of porous media. J. Petr. Tech. 12, p.1371-1378.

${ }^{2}$ Archie, G.E. 1942. The electrical resistivity $\log$ as an aid in determining some reservoir characteristics. Trans. AIME 146, p.55-62.

${ }^{3}$ Khilar, K.C., and Fogler, H.S. 1984. The existence of a critical salt concentration for particle release. J. Coll. Int. Sci. 101, p.214-224.

${ }^{4}$ King, M.S., Zimmerman, R.W., and Corwin, R.F. 1988. Seismic and electrical properties of unconsolidated permafrost. Geophys. Prosp. 36, p.349-364.

${ }^{5}$ Schlueter, E. 1991. The effect of clay on rock resistivity and the mechanisms affecting clay-fluid electrolytic conduction. Soil Surface and Colloid Chemistry Course (SS 221) Report, U.C. Berkeley.

${ }^{6}$ Schlueter, E., Myer, L.R., Cook, N.G.W., and Witherspoon, P.A. 1992. Relative permeability and the microscopic distribution of wetting and non-wetting phases in pore space of Berea sandstone. Lawrence Berkeley Lab. Report in progress.

${ }^{7}$ Schlueter, E., Myer, L.R., Cook, N.G.W., and Witherspoon, P.A. 1992. Formation factor and the microscopic distribution of wetting phase in pore space of Berea sandstone. Lawrence Berkeley Lab. Report in press.

${ }^{8}$ Sposito, G. 1984. The surface chemistry of soils. Oxford Univ. Press. N.Y.

${ }^{9}$ Suarez-Rivera, R. 1991. Personal communication.

${ }^{10}$ Williams, D.J.A., and Williams, K.P. 1978. Electrophoresis and zeta potential of kaolinite. J. Coll. Int. Sci. 65, p.79-87.

${ }^{11}$ Wyllie, M.R.J. 1963. The fundamentals of electric log interpretation. Acad. Press Inc. N.Y. 


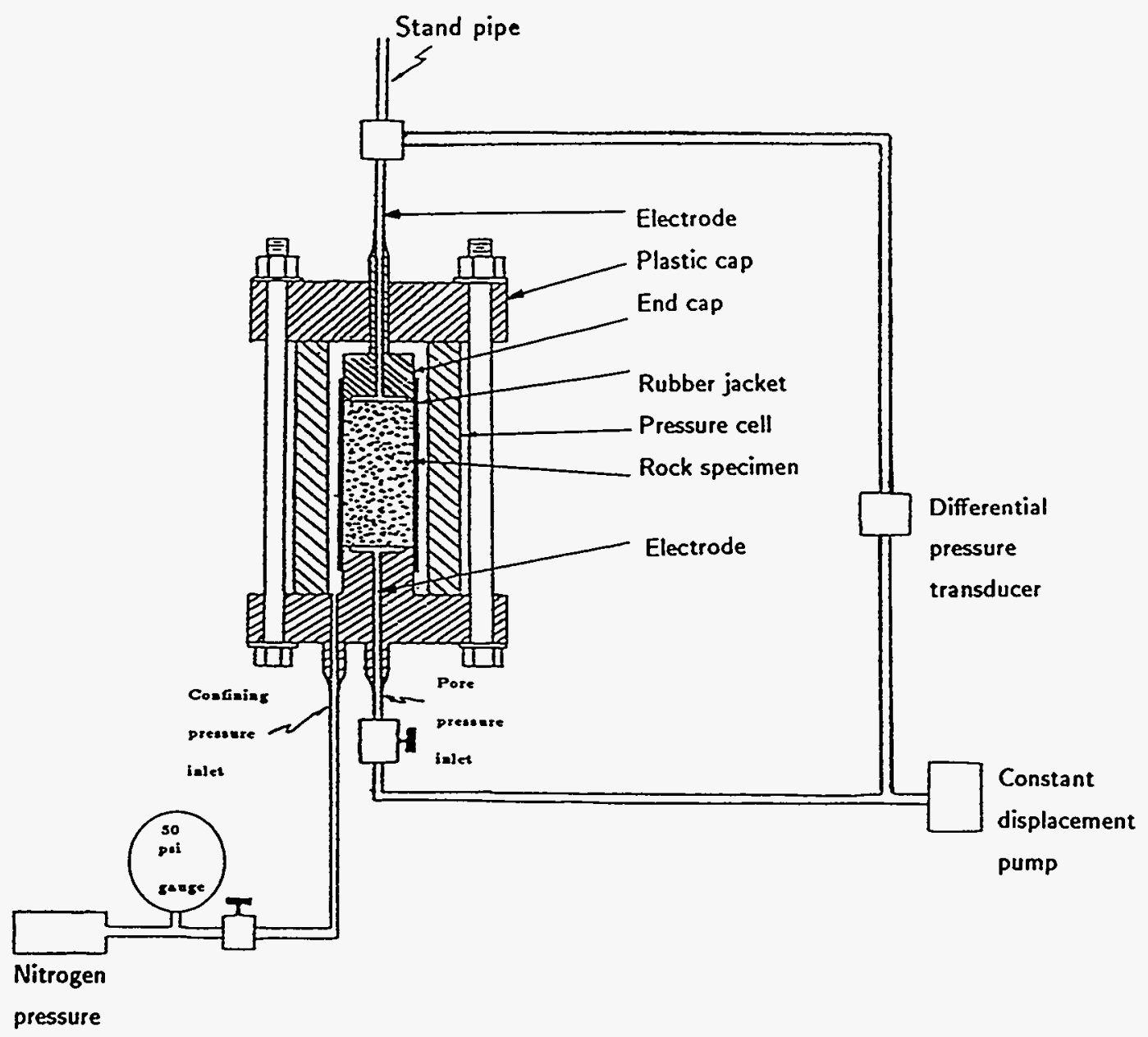

FIG. 6.1. Schematic representation of the laboratory apparatus designed to simultaneously measure both electric and hydraulic conductivity. 


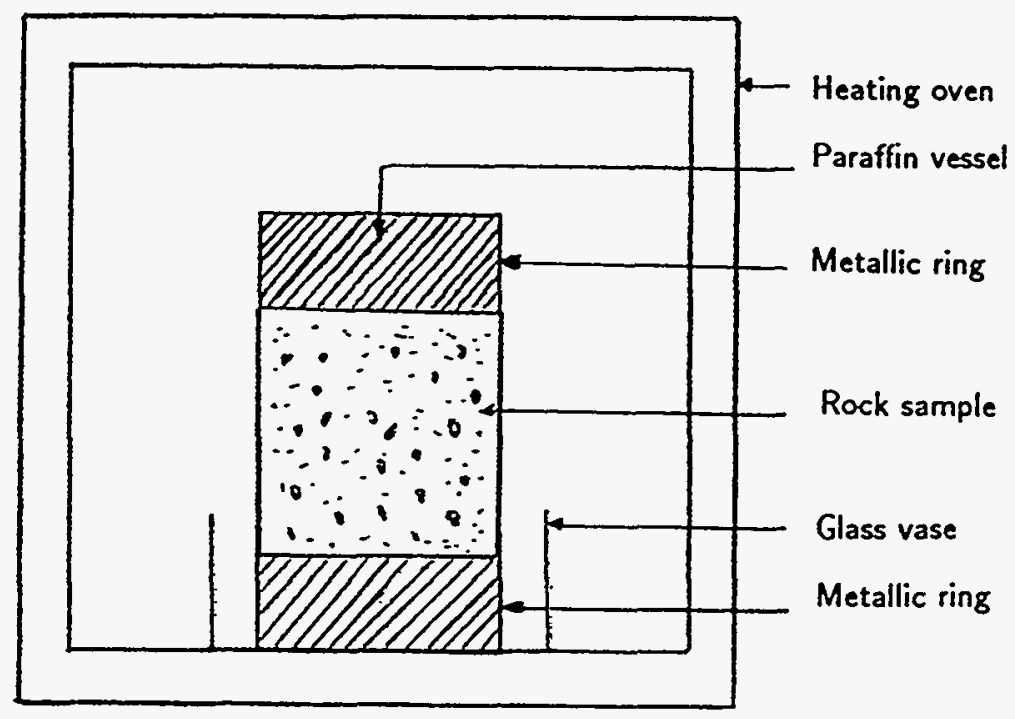

FIG. 6.2. Experimental setup for one-dimensional paraffin impregnation. 


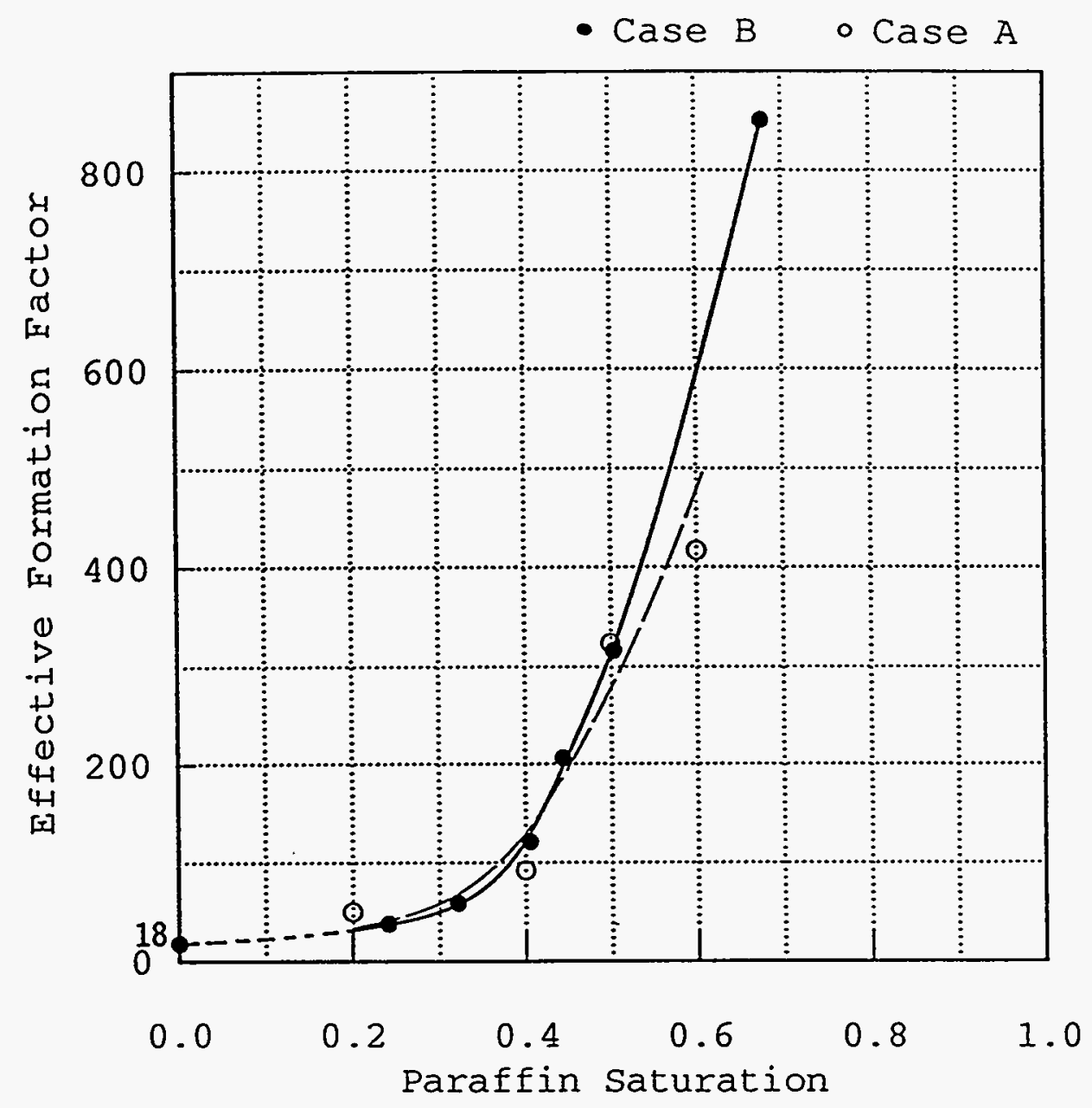

FIG. 6.3. Effective formation factor vs. hydrocarbon paraffin saturation for Berea sandstone. The pore space was partially saturated with hydrocarbon paraffin. The remaining portion of the pore space was filled with a $0.5 \mathrm{M}$ zinc nitrate solution. Cases A and B correspond to experimental data on samples subjected, or not subjected, to a formation factor measurement previous to paraffin application, respectively. 
-sureso zұsenb

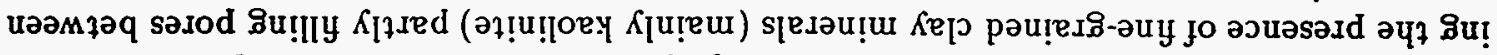

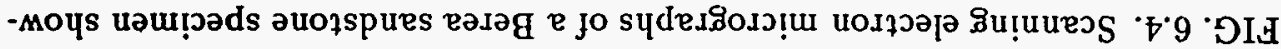
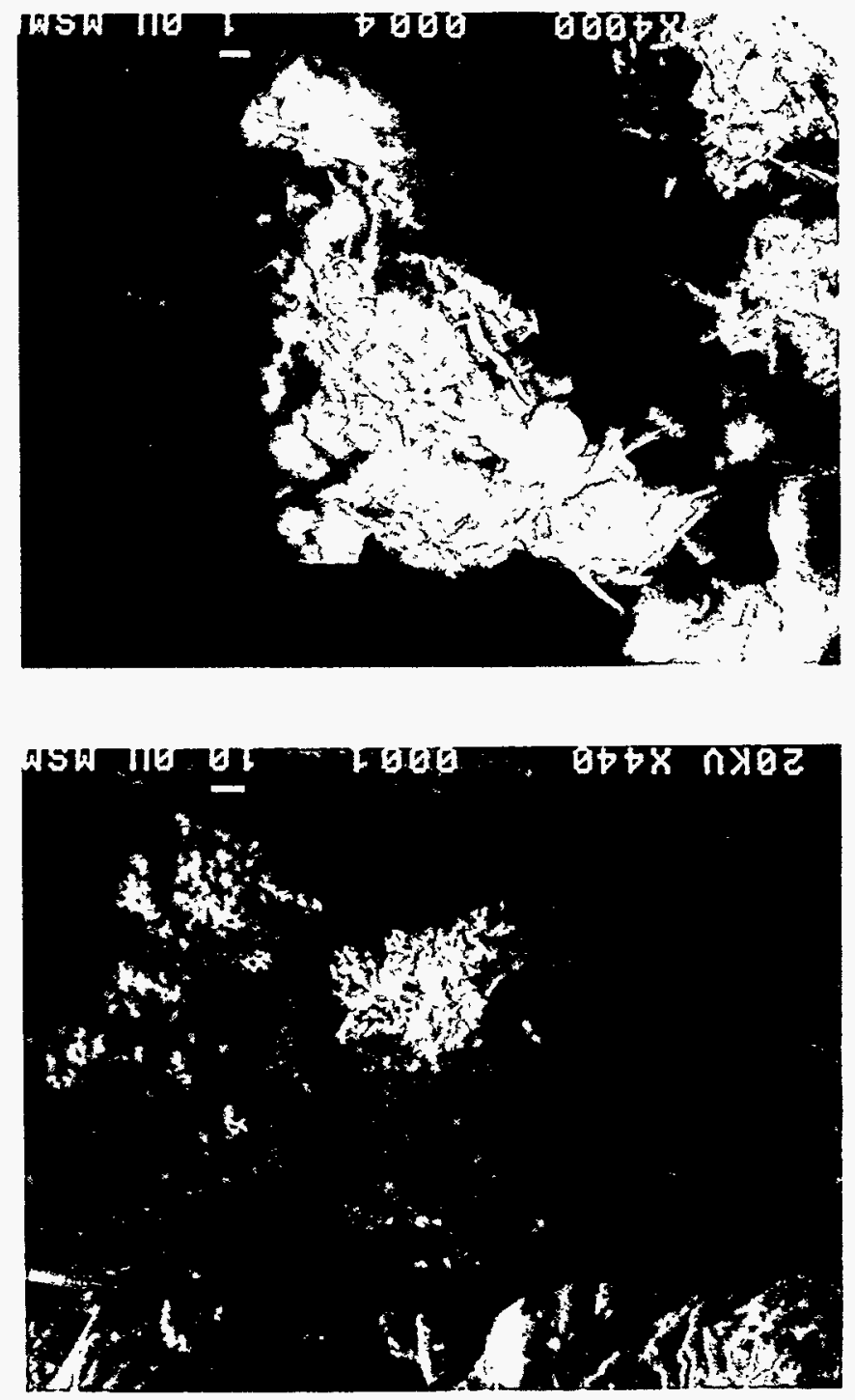


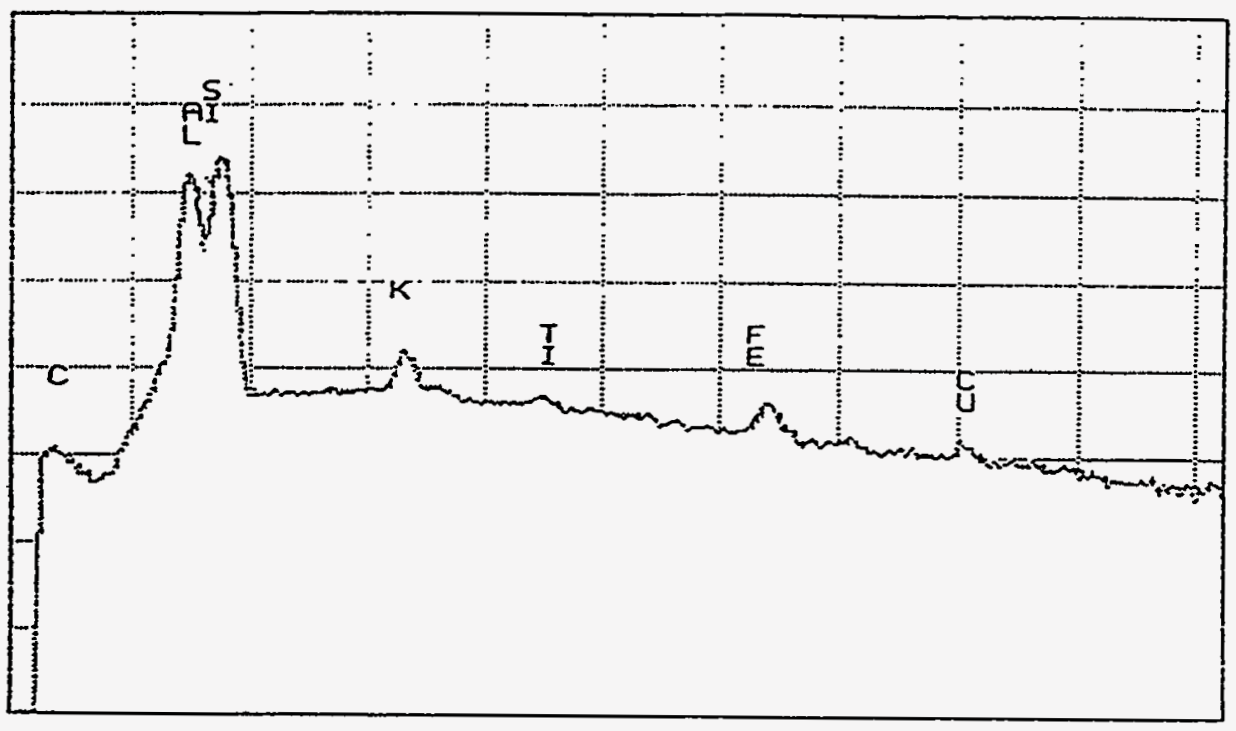

FIG. 6.5. X-ray spectrum of clay minerals coating Berea sandstone sample pores shown in figure 6.4. EDX analysis yielding nearly equal peak heights of $\mathrm{Si}$ and $\mathrm{Al}$ confirms the identification as kaolinite. 


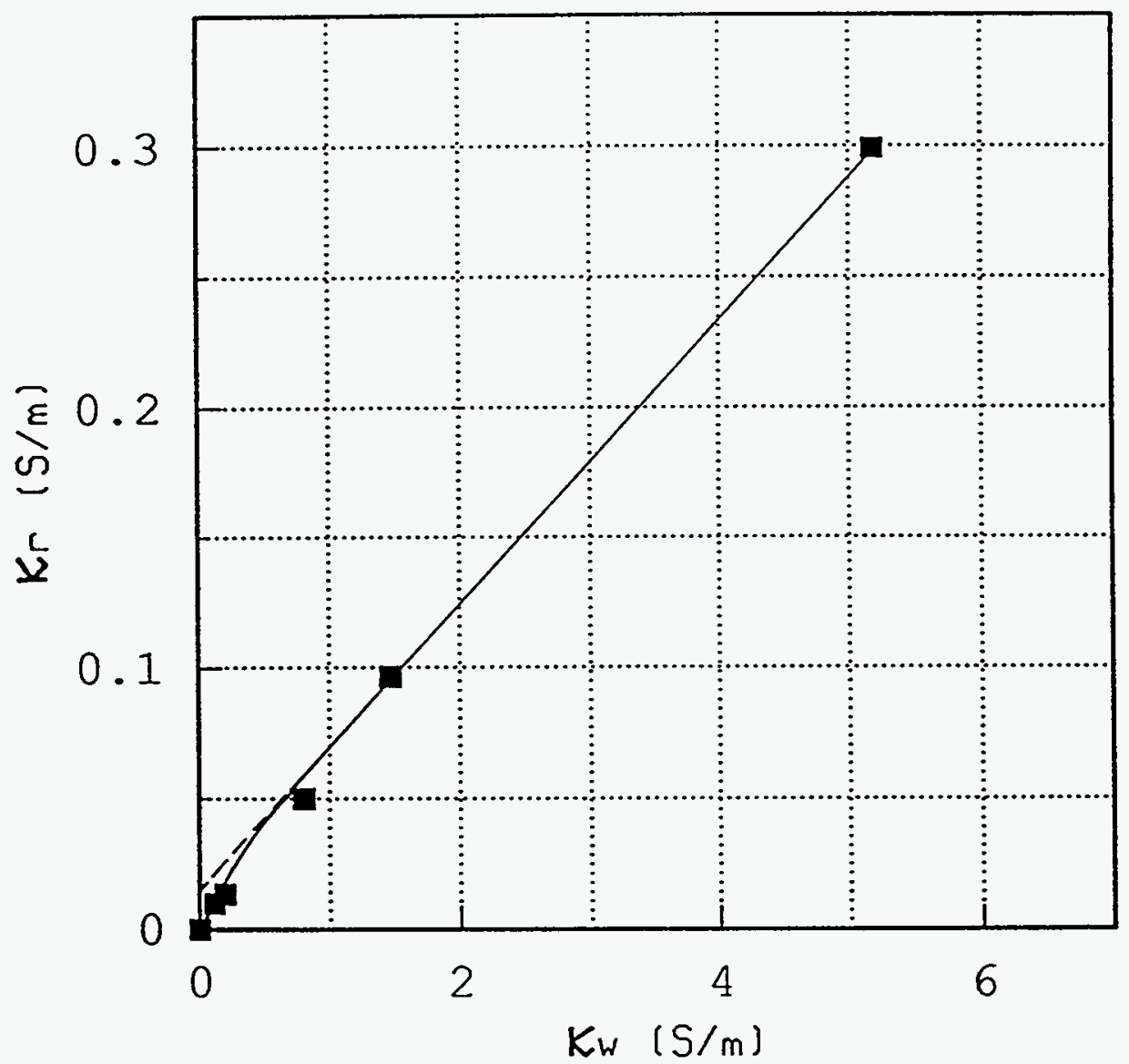

FIG. 6.6. Conductivity data of Berea sandstone at different electrolyte concentrations. Zinc nitrate electrolyte solutions with concentrations ranging from $0.005 \mathrm{M}$ to $0.5 \mathrm{M}$ were used. 


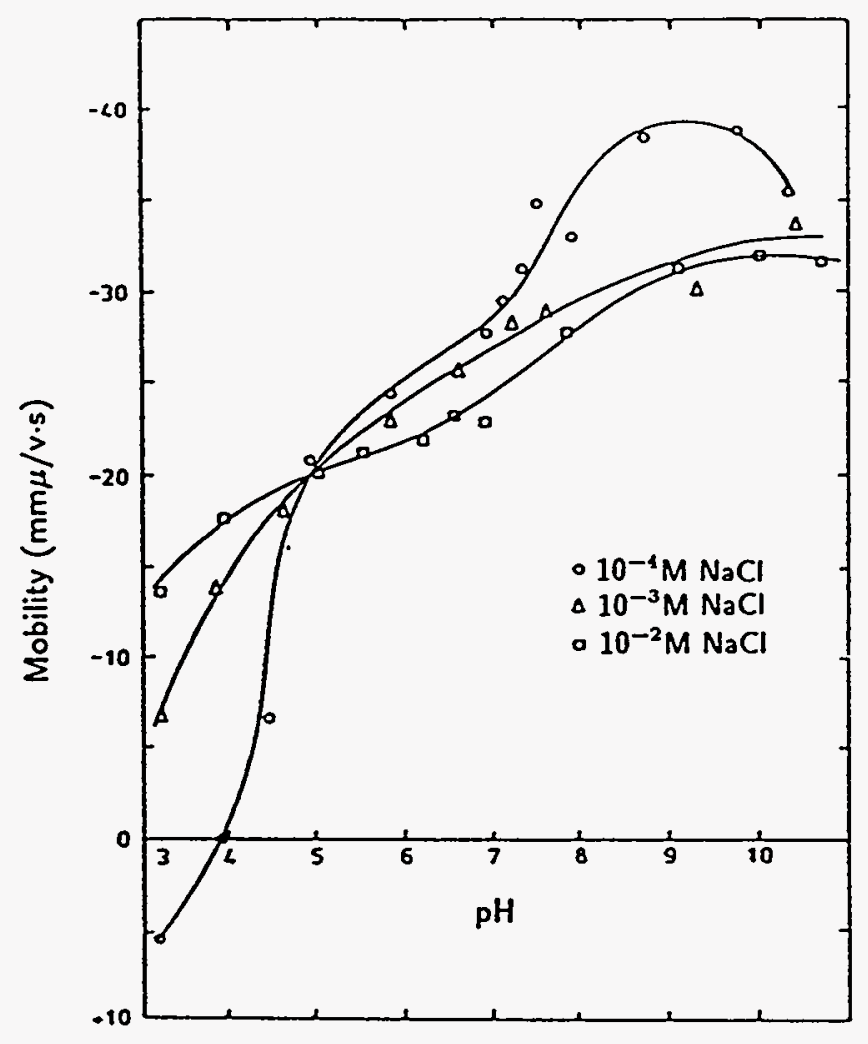

FIG. 6.7. Mobility for kaolinite as a function of salt concentration and $\mathrm{pH}$ (after Williams and Williams, 1978). 


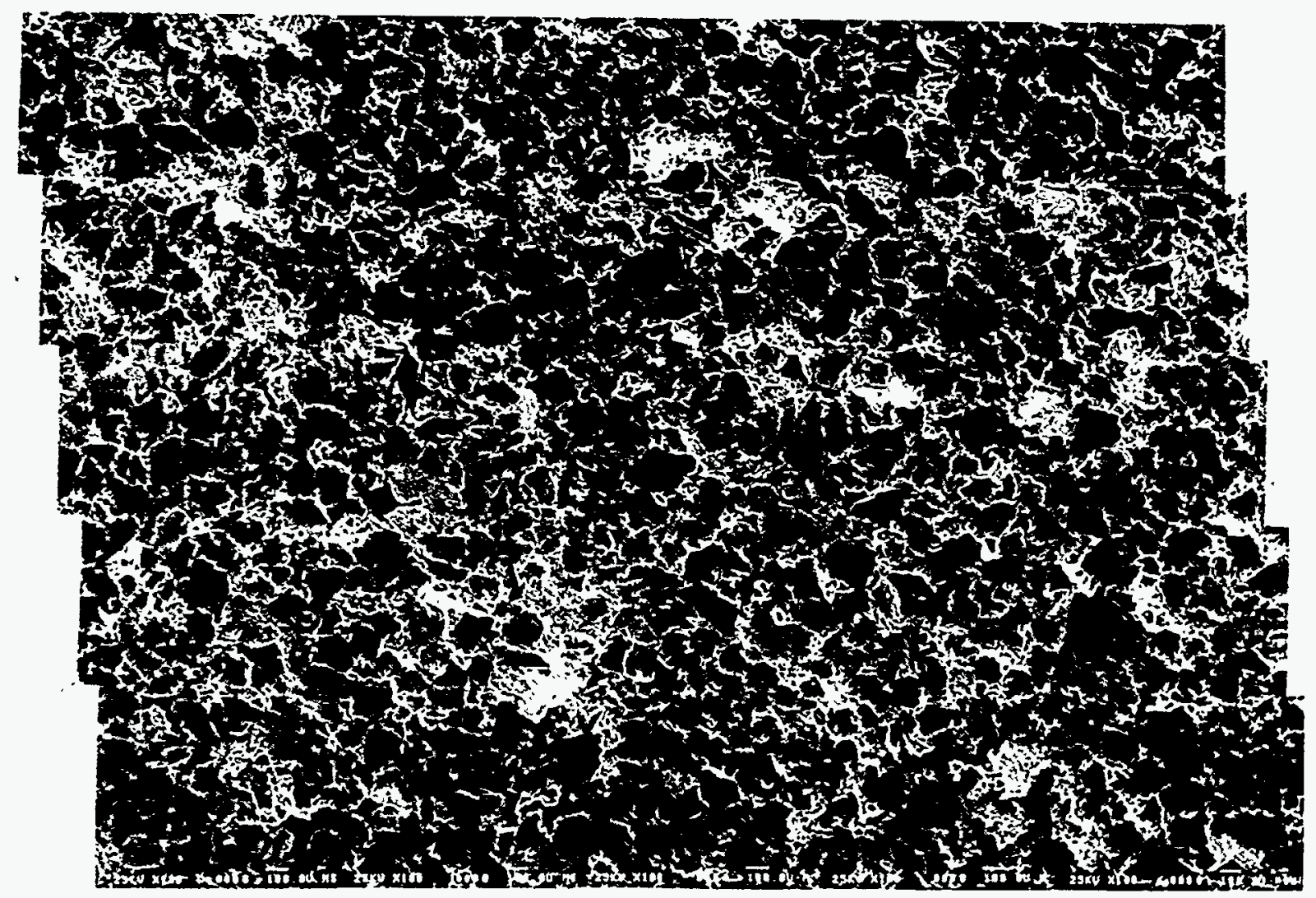

FIG. 6.8. Scanning electron micrograph collage of a Berea sandstone specimen impregnated with approximately $20 \%$ - 30\% paraffin. Actual width of field is about $6 \mathrm{~mm}$. The gray phase is quartz grains, the white phase is pores saturated with paraffin, and the black phase is remaining pores filled with blue epoxy for imaging purposes. 


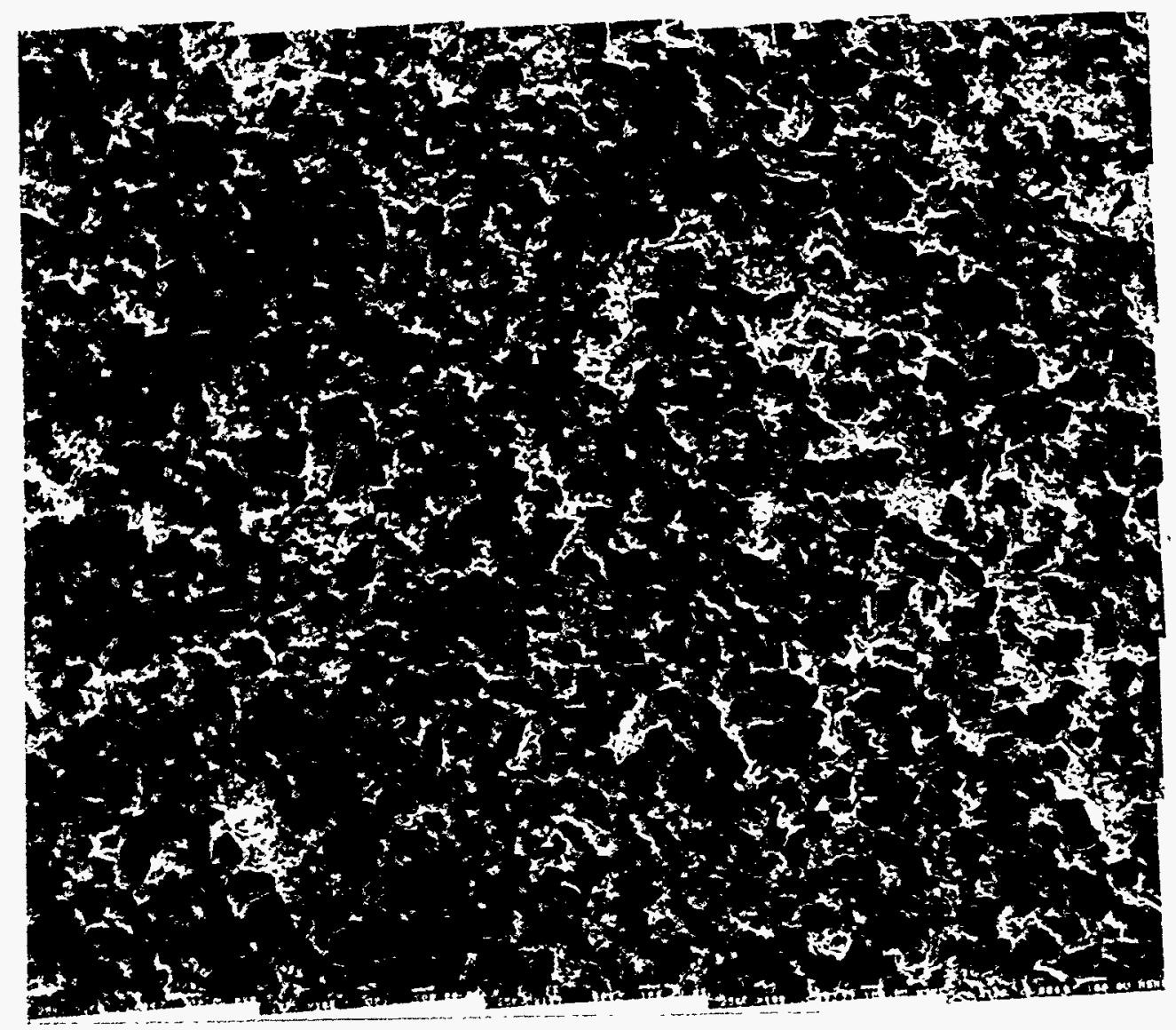

FIG. 6.9. Scanning electron micrograph collage of a Berea sandstone specimen impregnated with approximately $40 \%-50 \%$ paraffin. Actual width of field is about $6 \mathrm{~mm}$. The gray phase is quartz grains, the white phase is pores saturated with paraffin, and the black phase is remaining pores filled with blue epoxy for imaging purposes. 


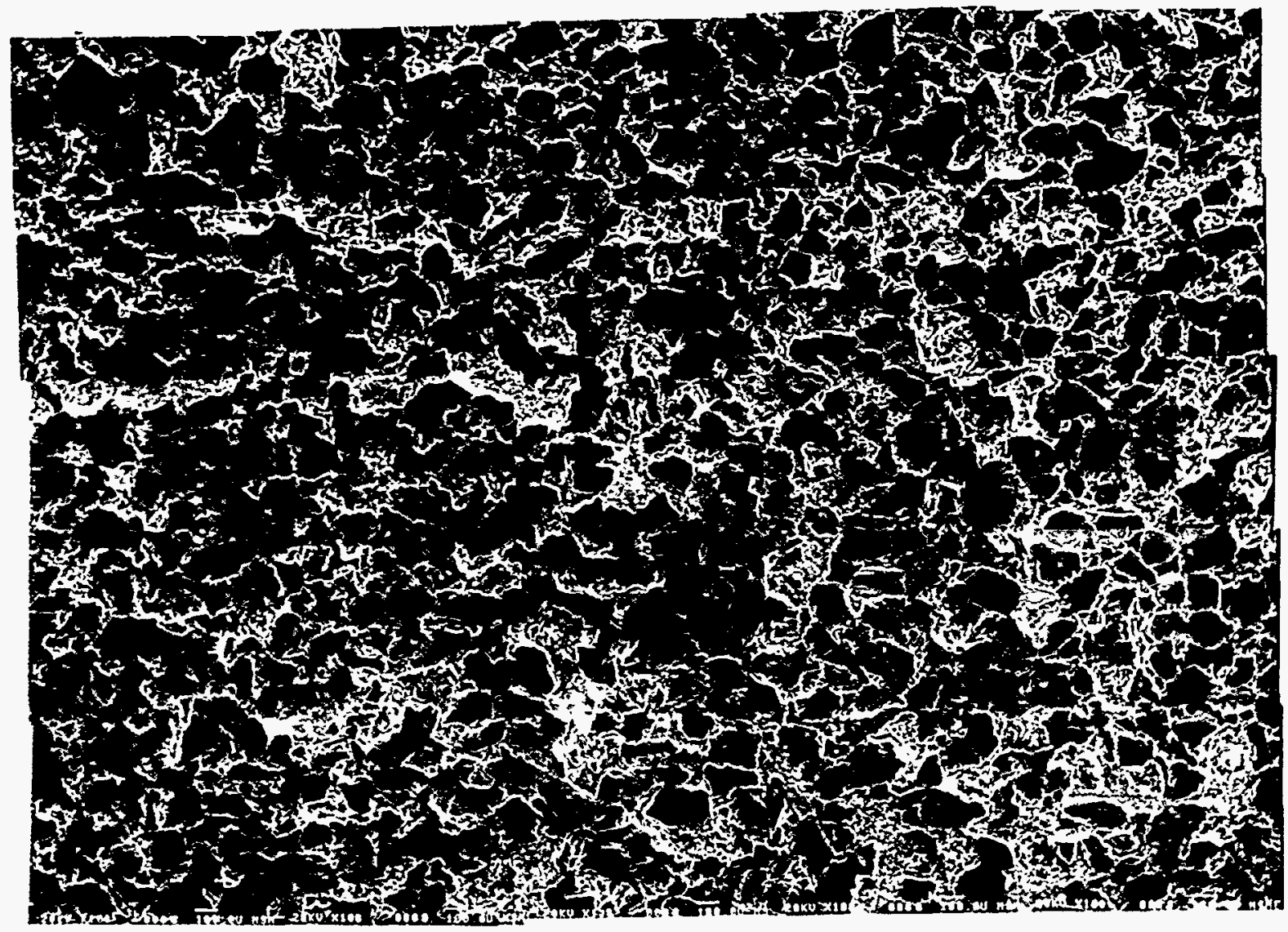

FIG. 6.10. Scanning electron micrograph collage of a Berea sandstone specimen impregnated with approximately $60 \%-70 \%$ paraffin. Actual width of field is about $6 \mathrm{~mm}$. The gray phase is quartz grains, the white phase is pores saturated with paraffin, and the black phase is remaining pores filled with blue epoxy for imaging purposes. 


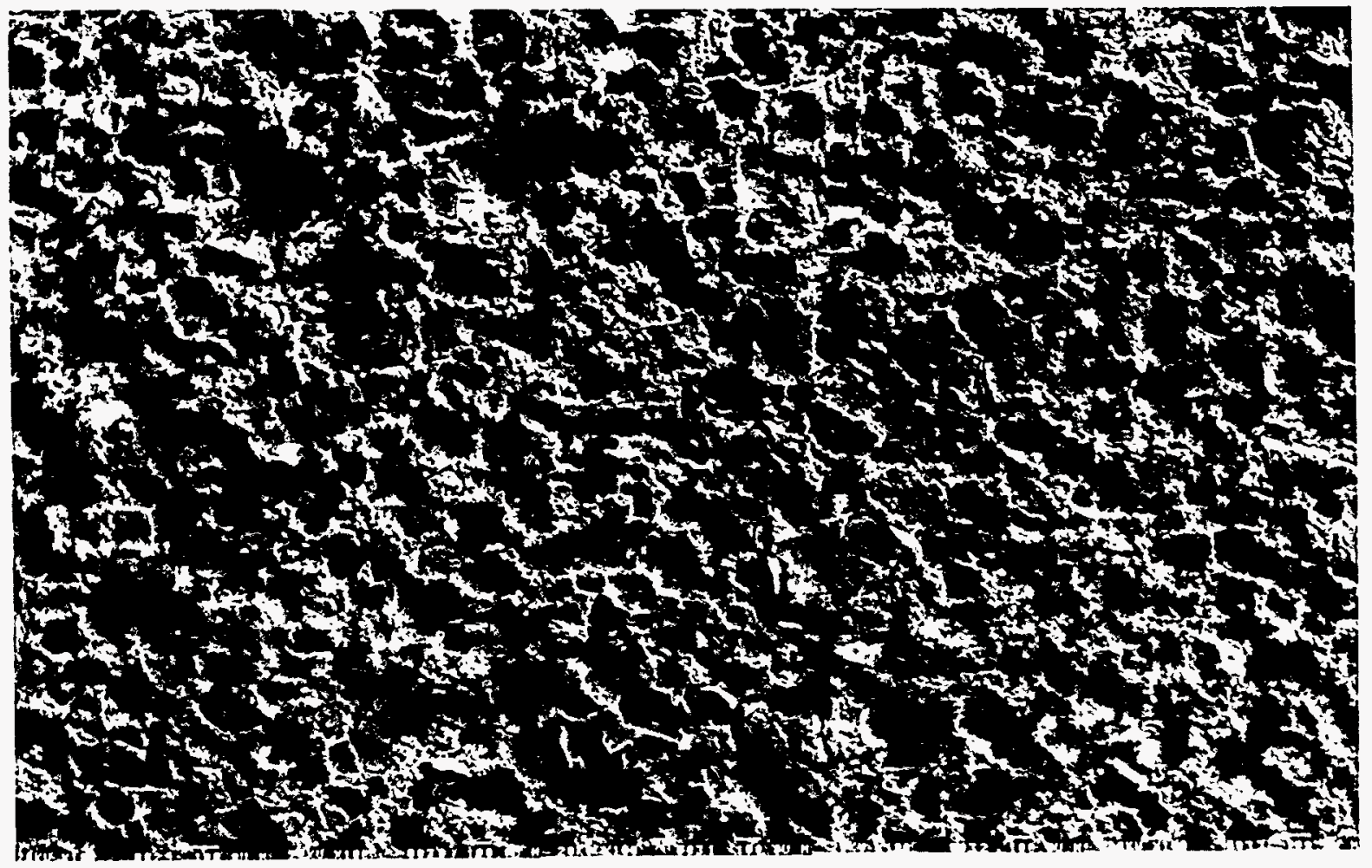

FIG. 6.11. Scanning electron micrograph collage of a Berea sandstone pore cast. Actual width of field is about $6 \mathrm{~mm}$. The rock pore space was completely filled with Wood's metal alloy and the quartz grains removed by hydrofluoric acid to allow direct observation of the pore structure. 


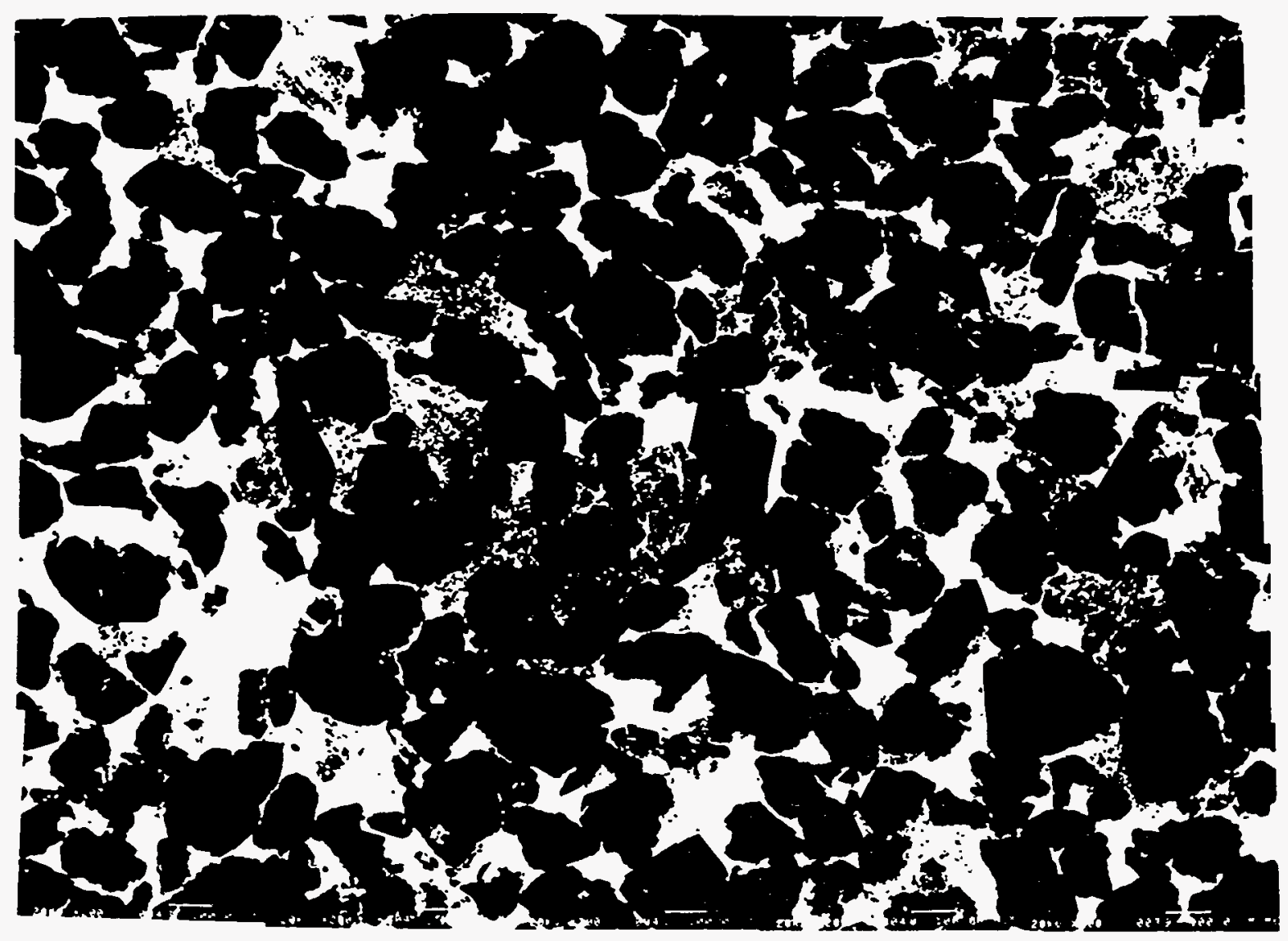

FIG. 6.12. Scanning electron micrograph collage of a Berea sandstone sample fully impregnated with Wood's metal alloy. Actual width of field is about $3 \mathrm{~mm}$. The gray phase is quartz grains, and the white phase is pores saturated with the alloy. The section reveals that the pore space is composed of grain contact (i.e., thin sheets and micropores), and intergranular porosity. 


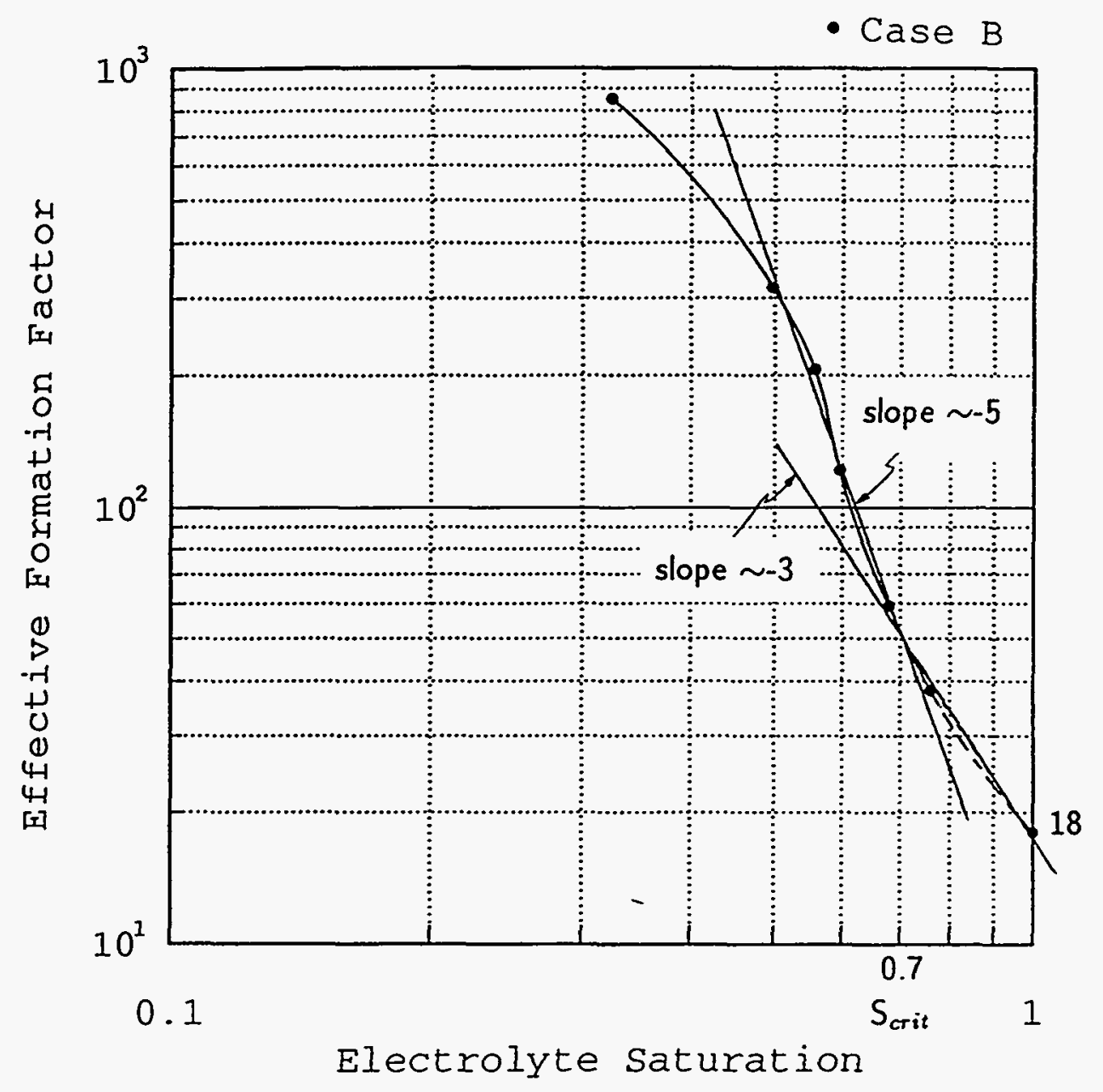

FIG. 6.13. Effective formation factor vs. electrolyte saturation for Berea sandstone (Case B). The pore space was partially saturated with hydrocarbon paraffin, with the remainder of the pore space filled with a $0.5 \mathrm{M}$ zinc nitrate solution. 
TABLE 6.1. Formation Factor Data - Berea Sandstone, with a $0.5 \mathrm{M}$ zinc nitrate solution used as electrolyte.

\begin{tabular}{ccc}
\hline \hline Sample & $\phi^{\mathrm{a}}(\%)$ & $\mathrm{F}^{\mathrm{b}}$ \\
\hline KS4 & 21.9 & 15.0 \\
KS6 & 22.6 & 14.3 \\
KS7 & 22.0 & 17.7 \\
KS9 & 22.2 & 15.9 \\
\hline \hline
\end{tabular}

${ }^{\text {a porosity. }}$

b formation factor.

TABLE 6.2. Resistivity Data - Berea Sandstone, with a zinc nitrate solution saturating the sample.

\begin{tabular}{cccc}
\hline \hline $\mathrm{M}^{\mathrm{a}}$ & $\mathrm{pH}$ (effluent) & $\rho_{w}^{\mathrm{b}}($ ohm $-\mathrm{m})$ & $\rho_{r}^{\mathrm{c}}($ ohm $-\mathrm{m})$ \\
\hline 0.5 & - & 0.19 & 3.35 \\
0.1 & - & 0.68 & 10.4 \\
0.05 & - & 1.24 & 20.1 \\
0.01 & 4.0 & 5.08 & 73.9 \\
0.005 & 4.5 & 9.01 & 101.3 \\
\hline \hline
\end{tabular}

a solution molarity.

b solution resistivity.

c rock resistivity. 


\section{PREDICTING THE CAPILLARY PRESSURE OF BEREA SANDSTONE FROM MICROGEOMETRY}

The macroscopic transport properties of porous (and fractured) media depend sensitively upon processes at the pore level, which are controlled principally by the geometry and connectivity of the pore space. In addition, the microphysics, microchemistry, and microbiology of processes at the pore level affect both the hydraulic and electric properties of porous materials. Visual observation and analysis of Berea sandstone pore structure via pore casts reveal that the rock total pore space is composed of grain contact pore space (i.e., thin sheets and micropores) connected to larger intergranular pore segments and pore throats, with the whole structure arranged in a three dimensional irregular network of irregularly shaped pores. Moreover, an exact description of microscopic texture of rocks is extremely complex, and generates too much data to be usefully handled. In this study, an attempt has been made to understand, through analysis and experiment, how the capillary pressure-saturation relationship is controlled by the rock structure and by the distribution of the nonwetting fluid in pores.

In our investigation, pore microgeometry based analytical calculations of the capillary pressure have been conducted. As a zero-order approximation, we have idealized the porous medium as consisting of an assembly of parallel capillaries of arbitrary cross-sections. Two-dimensional scanning electron microscope (SEM) photomicrographs of rock cross-sections have been employed to infer the areas, perimeters, and the hydraulic radii of the individual pores. The mathematical expression for the capillary pressure as a function of saturation curve depends on the distribution of pore hydraulic radii and the area-perimeter power-law relationship (Schlueter et al., 1992a). These quantities have been measured directly from two-dimensional SEM photomicrographs of rock sections. Account is taken of the fact that the cross-sections are randomly oriented with respect to the channel axes. The predictions of our model are compared to laboratory capillary pressure curves obtained using Wood's metal alloy as the nonwetting phase instead of the conventional mercury porosimetry. This technique allows for direct examination and analysis of the fluid distributions in the rock pore space after the experiment (obtained at fixed equilibrium pressures and fluid saturations).

\section{A. The model}

In two-phase conditions, the capillary pressure between wetting and nonwetting phases in a circular tube of radius $r$ is given by Laplace's equation (Scheidegger, 1974) 


$$
P_{c}=\frac{2 \varsigma \cos \alpha}{r}
$$

where $\varsigma$ is the surface tension between wetting and nonwetting phases, and $\alpha$ the contact angle between the wetting phase meniscus and the tube wall.

If the capillaries are not circular, the equation for the capillary pressure has to be generalized by replacing $2 / r$ by $1 / r_{1}+1 / r_{2}$

$$
P_{c}=\varsigma\left(\frac{1}{r_{1}}+\frac{1}{r_{2}}\right)
$$

where $r_{1}$ and $r_{2}$ are the principal radii of curvature of the meniscus.

If the pore-openings are not of a simple geometric form, Eq. 33 is still a valid expression for the capillary pressure. In order to obtain a theoretical relationship between the saturation and capillary pressure for a porous medium, an analytical expression for the average interfacial curvature as a function of saturation has to be found. This is a very difficult task.

Schultze $(1925 \mathrm{a}, \mathrm{b})$ has shown that the capillary pressures for such capillaries under the assumption of zero contact angle are given approximately by the equation

$$
P_{c}=\frac{\varsigma}{R_{H}}
$$

where $R_{H}$ is the ratio of area to perimeter of the capillary. A list of comparative values to test Eq. 34 is given in Table 7.1 (after Carman, 1941).

Since Eq. 34 gives a reasonably accurate prediction of capillary pressure in noncircular capillaries, it can be assumed to be applicable to the capillary channels in a porous medium (Scheidegger, 1974).

In our model, it is assumed that there is no accessibility problem, i.e. regardless of the spatial arrangement of pores, the pores are occupied by the nonwetting phase in the order of largest pores first. Similarly, for the wetting phase, the pores are occupied by the wetting phase in the order of smallest pores first. Therefore, given a hydraulic radius distribution of intergranular pore space $\beta\left(R_{H}\right)$, and assuming that the pores are filled by the wetting phase in ascending order up to a cut-off radius $R_{H}^{\prime}$, we may write the saturation of the wetting phase $S_{w}\left(R_{H}^{\prime}\right)$ as (cf., Pruess and Tsang, 1989; Schlueter and Pruess, 1990)

$$
S_{w}\left(R_{H}^{\prime}\right)=\frac{\int_{0}^{R_{H}^{\prime}} A\left(R_{H}\right) \beta\left(R_{H}\right) d R_{H}}{\int_{0}^{\infty} A\left(R_{H}\right) \beta\left(R_{H}\right) d R_{H}}
$$


where the pore hydraulic radius is defined as the ratio of the pore area $A$ to the pore perimeter $P$.

Measurements of hydraulic radius of intergranular pore space obtained from twodimensional SEM photomicrographs of Berea sandstone rock sections have been found to follow a skewed distribution well approximated by a log-normal distribution. The log-normal distribution is given by the following expression (hydraulic radius $R_{H} \geq 0$ )

$$
\beta\left(R_{H}\right)=\frac{1}{\sqrt{2 \pi} \sigma \ln 10} \frac{1}{R_{H}} \exp \left(\frac{-\left[\log R_{H}-\log R_{H^{\circ}}\right]^{2}}{2 \sigma^{2}}\right)
$$

where $R_{H_{0}}$ is the most probable hydraulic radius, and $\sigma$ the variance. The corresponding mean hydraulic radius $R_{H_{m}}$ is larger than the most probable hydraulic radius; it is

$$
R_{H_{m}}=R_{H_{0}} \exp \left(\frac{(\sigma \ln 10)^{2}}{2}\right)
$$

Invoking the perimeter-area power-law relationship (Schlueter et al., 1992a)

$$
A=m P^{\gamma}
$$

where $\log m$ is the intercept on the $\log A$ axis, and $\gamma$ the non-integer slope of the $\log A-\log P$ plot, the area can be expressed in terms of the hydraulic radius as follows

$$
A\left(R_{H}\right)=m^{\frac{1}{1-\gamma}} R_{H}^{\frac{\gamma}{\gamma-1}}
$$

Expressed in terms of the cut-off hydraulic radius, Eq. 35 becomes

$$
S_{w}\left(R_{H}^{\prime}\right)=\frac{\int_{0}^{R_{H}^{\prime}} R_{H}^{\frac{\gamma}{\gamma-1}} \beta\left(R_{H}\right) d R_{H}}{\int_{0}^{\infty} R_{H}^{\frac{\gamma}{\gamma-1}} \beta\left(R_{H}\right) d R_{H}}
$$

Integrating Eq. 40 yields

$$
S_{w}\left(R_{H}^{\prime}\right)=\frac{1}{2}\left(1+\operatorname{erf}\left[\frac{\left(\log R_{H}^{\prime}-\delta\right)}{\sqrt{2 \sigma^{2}}}\right]\right)
$$

where the cut-off hydraulic radius is given by

$$
R_{H}^{\prime}=10^{\delta-\sqrt{2} \sigma e r f i\left(1-2 S_{w}\right)}
$$

where $\delta=\sigma^{2} \ln 10\left(\frac{\gamma}{\gamma-1}\right)+\log R_{H_{0}}, \sigma$ is the variance of $\log R_{H}$, and erf, erfi are the error and the inverse error function, respectively. The capillary pressure is then given by 


$$
P_{c}=\frac{\varsigma}{R_{H}^{\prime}}=\varsigma 10^{\sqrt{2} \sigma e r f i\left(1-2 S_{w}\left(R_{H}^{\prime}\right)\right)-\delta}
$$

Capillary pressure is specific to the nature of the two fluids involved. If no specification is made, it is understood that the displaced fluid is vacuum. If the displaced fluid is a vacuum, and the external pressure $P_{c}$ is applied in a nonwetting fluid, then all câpillaries with with a radius larger than $R_{H}^{\prime}$ will be totally filled.

\section{B. Correction for pore orientation}

In the two-dimensional sections under consideration, however, the pore crossections are randomly oriented with respect to the directions of the channel axes. The orientation effect has been corrected by means of the following geometrical and stereological considerations (cf., Underwood, 1970), which are exact for the case of circular cross-sections.

For the hydraulic radius,

$$
\left(R_{H}\right)_{\text {actual }}=\frac{\sqrt{2}}{2}\left\langle\frac{1}{\sqrt{1+\cos ^{2} \theta}}\right\rangle^{-1}\left(R_{H}\right)_{\text {measured }}
$$

where the brackets denote a spherical average for pore of random orientation, i.e.,

$$
\left\langle\frac{1}{\sqrt{1+\cos ^{2} \theta}}\right\rangle=\frac{\int_{0}^{\pi} \int_{0}^{\theta_{\max }} \frac{a^{2} \sin \theta}{\sqrt{1+\cos ^{2} \theta}} d \theta d \phi}{\int_{0}^{\pi} \int_{0}^{\theta_{\max }} a^{2} \sin \theta d \theta d \phi}
$$

with $\theta_{\max }=\arctan (L / D)$, where $(L / D)$ is the maximum ratio of pore length to diameter. Using an average value of $L / D=5$, as estimated from the micrographs, we find that

$$
\left(R_{H}\right)_{\text {actual }}=0.85\left(R_{H}\right)_{\text {measured }}
$$

\section{The experiment}

In this experimental investigation, we have sought to examine the relationship between the microscopic pore occupancy by the nonwetting fluid and its effect on capillary pressure.

We have used three-dimensional imbibition of a nonwetting Wood's metal alloy instead of the conventional mercury porosimetry. This technique offers the advantage of allowing the analysis of the occupied pore space after the experiment. Wood's metal is an alloy of about $43 \% \mathrm{Bi}, 38 \% \mathrm{~Pb}, 11 \% \mathrm{Sn}$, and $9 \% \mathrm{Cd}$, with a specific gravity of 9.6 , a viscosity of about $1.3 \times 10^{-3} \mathrm{~Pa} \cdot \mathrm{s}$ at $75^{\circ} \mathrm{C}$, and a surface tension of about $400 \mathrm{mN} / \mathrm{m}$ 
(Yadav et al., 1987). The setup for the three-dim:nsional imbibition experiments is shown in Fig. 7.1. It consists of a metallic container of Wood's metal placed in a metal vacuum chamber provided with a lucite window and surrounded by a heating element to keep the metal molten (melting point varies from about $50^{\circ} \mathrm{C}$ to $70^{\circ} \mathrm{C}$ depending on its composition). A micrometer is attached to the metallic container to determine the pressure at which the Wood's metal first enters the specimen. The $50 \mathrm{~mm}$-long and $50 \mathrm{~mm}$-diameter sandstone sample is first oven dried, and then immersed in the molten Wood's metal in the metallic container and placed in the metal vacuum chamber. Then the sample is de-aired by applying a full vacuum for about 60 minutes, until no air bubbles are observed through the lucite window. Capillary pressure is applied by drawing a partial vacuum, which is maintained at the desired value by a regulating valve. Each sample was allowed to imbibe at a fixed equilibrium pressure for approximately 90 minutes and until no movement of Wood's metal was noticed. At a pressure of about 5 psia to 6 psia, the micrometer signaled the first indication of Wood's metal entering the pore space (probably an edge effect on the sample sides). The capillary pressure experiment was repeated on several samples by applying pressure in the range of approximately 6 psia to 14 psia. The imbibed samples were cut into four axial quarters each of which had a different saturation. To minimize the effect of gravity (hydrostatic) gradient, we took the top quarter of each imbibed specimen at a particular equilibrium pressure and measured its saturation. The capillary pressure curve obtained when partially saturating the rock with the nonwetting fluid is presented in Fig. 7.2. There is a sharp increase in nonwetting fluid saturation with a corresponding small increase in capillary pressure in the saturation range up to about $50 \%$. Our result is consistent with typical capillary pressure curves for Berea (BE-1) sandstone reported in the literature (Fig. 7.3), using conventional mercury porosimetry saturation (Chatzis and Dullien, 1977). Berea (BE1) sandstone has almost the same macroscopic properties as the Berea sandstone we used in our experiments (e.g., porosity of $22 \%$, permeability to $\mathrm{N}_{2}$ of $400 \mathrm{md}$, and a formation factor of 15.5). Berea sandstone is a homogeneous sedimentary rock used as a reference rock in the petroleum industry. It contains about $80 \%$ quartz, $12 \%$ feldspar, and $8 \%$ by weight of dispersable and swelling clays (mainly kaolinite, with some illite and smectite). It is estimated to be of Missisipian age and is found in Berea, Ohio.

\section{Effect of pore structure and topology}

To understand how pore structure and topology control the physical property of consideration, the capillary pressure data has been studied in light of the nonwetting fluid distributions observed at each equilibrium pressure. For this purpose, optical 
and scanning electron microscopic examinations of samples tops (after cutting off 3 $\mathrm{mm}$ ) have provided valuable insights into the pore level complexity of the natural porous media. An optical photograph of the fluid distributions obtained in top axial quarters (top and bottom) in the pressure range 6.8 psia to $7.7 \mathrm{psia}$ is presented in Fig. 7.4. It is observed that the nonwetting fluid flow network is composed of a set of imbibing clusters correlated in space. At every pressure step, the nonwetting fluid resides in the pores accessible through throats with a radius larger than that corresponding to the current equilibrium capillary pressure. As the pressure increases, the nonwetting phase saturation increases and the nonwetting fluid invades successively smaller pores and becomes connected to regions which were separated from this phase by small throats. Optical photograghs of enlarged fluid distributions in top axial quarters obtained by partially saturating the rock with Wood's metal at equilibrium pressures of 6.8 psia, 6.9 psia, and 7.2 psia are presented in Figs. 7.5, 7.6, and 7.7, respectively. At 6.8 psia (Fig. 7.5), the fluid has preferentially penetrated the sample sides. The saturation is greatest near the perimeter of the sample and least at the center. This observation suggests that pores near the cylindrical surface of the sample are better connected than those towards the center. This interconnection could arise from the presence of the surface, which exposes all pores intersected by it, or from damage adjacent to this surface. At 6.9 psia (Fig. 7.6), a saturation gradation is observed in the direction of flow at this pressure (preferentially horizontal). The longer flow paths are connected by smaller constrictions, so fewer flow channels are going to the sample center starting from all available channels at sample surface. At pressures of 7.2 psia (Fig. 7.7) and greater, the nonwetting fluid invades more and more smaller pores, becoming connected to regions that were separated to this phase by smaller pores, and the clusters of nonwetting phase become larger and larger. A SEM photomicrograph collage of a 1 in $\times 1$ in rock specimen saturated with approximately $50 \%$ of Wood's metal at 8.5 psia equilibrium pressure is presented in Fig. 7.8. A SEM photomicrograph collage of an enlarged partial section from the last figure and its associated pore contours areas (Fig. 7.9), show that there are many large (intergranular) pore segments connected by small throats that do not contribute to the flow of the nonwetting phase in the rock. In addition, the grain contact pore space (i.e., thin sheets and micropores) does not contribute either. Furthermore, simple statistical analysis of pore contour areas obtained from Fig. 7.9 has shown that relatively few conduits connected by large intergranular throats carry up to about $50 \%$ of the nonwetting fluid in the porous media under consideration, producing a clustered structure.

\section{E. Applications}


On this section, we calculate the predicted capillary pressure function for Berea sandstone for comparison with our experimental data. A typical scanning electron microscope photomicrograph of Berea sandstone is presented in Fig. 7.10. Computerized analysis of Berea sandstone image shows contours of intergranular pore space (Fig. 7.11) from which the perimeter-area power-law relationship of pores, (Fig. 7.12) and the distribution of pore hydraulic radii have been obtained (Fig. 7.13). It is worth noting that the contours of intergranular pore space shown in Fig. 7.11 are the same contours from which the transport properties (hydraulic and electric) have been previously calculated (Schlueter et al., 1991; 1992a). The analytical capillary pressure function for Berea sandstone has been computed using $\varsigma=0.40 \mathrm{~N} / \mathrm{m}$ (surface tension), $\gamma=1.49$ (perimeter-area power-law non-integer slope), $R_{H_{m}}=12.8 \mu \mathrm{m}$ (mean hydraulic radius), $\sigma=0.41$ (standard deviation), and $\left(R_{H}\right)_{\text {actual }} /\left(R_{H}\right)_{\text {measured }}=0.85$ (stereological correction). Preliminary results are presented in Fig. 7.14. As seen, the predicted capillary pressure function from such a hydraulic radius distribution does not fit the experimental capillary pressure. To fit the analytical to the experimental function at approximately $50 \%$ fluid saturation, a distribution represented by $R_{H_{m}}=6.6 \mu \mathrm{m}$, and $\sigma=0.14$ is needed. The mean hydraulic radius, $R_{H_{m}}=6.6 \mu \mathrm{m}$, corresponds to the mean hydraulic radius of the intergranular throats of the medium, possibly the same throats that largely control hydraulic conductivity (Schlueter et al., 1991). Our preliminary analyses then show that the experimental capillary vs. saturation function (in the saturation range up to about $50 \%$ ) is controlled primarily by intergranular throats of narrow size distribution, represented by a mean hydraulic radius of about $6.6 \mu \mathrm{m}$, and a standard deviation of 0.14 .

\section{F. Concluding remarks}

Pore size distributions and pore sizes of rocks have been measured by many researchers using a variety of methods (Dullien and Dhawan, 1974, 1975; Chatzis and Dullien, 1982; Chatzis et al., 1983; Yanuka et al., 1986; Jerauld and Salter, 1990). Most methods require a model of the pore space and simplifying assumptions. Because of these difficulties and the simplified model we use, we have not attempted to precisely determine pore-size distribution but use a simple functional form to determine average pore and throat sizes controlling capillary pressure. Our result, assuming a lognormal distribution of pore sizes, is consistent with the one obtained by Jerauld and Salter (1990) using an exponential pore-size distribution for Berea sandstone, and similar to that used by Chatzis and Dullien (1982). Jerauld and Salter (1990) also found that pore-throats are smaller than pore-bodies; $r_{t_{m}}=11 \mu \mathrm{m}$ and $r_{b_{m}}=38$ $\mu \mathrm{m}$ (Fig. 7.15). It should be noted that the simple concept leading to Eq. 43 has several limitations, so the result is only a first approximation. For example, we have 
ignored possible effects from the wetting phase being held by small scale roughness and adsorptive forces in the pore walls, we have neglected the effect of clay minerals, which complicates phase occupancy and mobility. Finally, the model does not account for hysteresis effects originated from different pore accessibilities during drying and wetting cycles.

Based on our experimental observations of relationship between microscopic nonwetting fluid occupancy, the fluid distributions, and their effect on capillary pressure of Berea sandstone, we have found that relatively few channels connected by large intergranular throats conduct a relatively large amount (up to about 50\%) of the nonwetting fluid through the medium. In fact, we have also found that a large percentage of the permeability of the medium is contributed by relatively few conduits connected by large intergranular throats, of narrow size distribution, and of high hydraulic conductance (Schlueter et al., 1992b).

${ }^{1}$ Carman, P.C. 1941. Soil Sci. 52,I.

${ }^{2}$ Chatzis, I., and Dullien, F.A.L. 1977. J. Can. Pet. Technol. 16,97.

${ }^{3}$ Chatzis, I., and Dullien, F.A.L. 1982. Application of the theory of percolation for a model of drainage in porous media and relative permeability of injected nonwetting liquid. Rev. l'Institut Francais du Petrole 37, p.183-205.

${ }^{4}$ Chatzis, I., Morrow, N.R., and Lim, H.T. 1983. Magnitude and detailed structure of residual oil saturation. SPEJ, April, p.311-326.

${ }^{5}$ Dullien, F.A.L., and Dhawan, G.K. 1974. Characterization of pore structure by a combination of quantitative photomicrography and mercury porosimetry. J. Coll. Int. Sci. 47, p.337-349.

${ }^{6}$ Jerauld, G.R., and Salter, S.J. 1990. The effect of pore structure on hysteresis in relative permeability and capillary pressure: pore-level modeling. Transport in Porous Media 5, p.103-151.

${ }^{7}$ Pruess, K., and Tsang, Y.W. 1989. On relative permeability of rough-walled fractures. Lawrence Berkeley Laboratory Report, LBL-26509, Berkeley, CA.

${ }^{8}$ Scheidegger, A.E. 1974. The physics of flow through porous media. University of Toronto Press. 
${ }^{9}$ Schlueter, E., and Pruess, K. 1990. Sensitivity studies on parameters affecting gas release from an underground rock cavern. Lawrence Laboratory Report, LBL-28818, Berkeley, CA.

${ }^{10}$ Schlueter, E., Zimmerman, R.W., Cook, N.G.W., and Witherspoon, P.A. 1991. Predicting permeability and electrical conductivity of sedimentary rocks from microgeometry. Proceedings, $32^{\text {nd }}$ U.S. Symposium on Rock Mechanics, Balkema, Rotterdam.

${ }^{11}$ Schlueter, E., Zimmerman, R.W., Cook, N.G.W., and Witherspoon, P.A. 1992a. Perimeter-area power-law relationship of pores in sedimentary rocks and implications for permeability. Lawrence Berkeley Laboratory-1992 Earth Sciences Division Annual Report, LBL-31500, Berkeley, CA. Presented at the Soc. Eng. Sci. $29^{\text {th }}$ Tech. Conf., San Diego, CA, September, 1992.

${ }^{12}$ Schlueter, E., Myer, L.R., Cook, N.G.W., and Witherspoon, P.A. 1992b. Relative permeability and the distribution of wetting and nonwetting phases in pore space of Berea sandstone. Lawrence Berkeley Lab. Report in progress. Presented at the Soc. Eng. Sci. $29^{\text {th }}$ Tech. Conf., San Diego, CA, September.

${ }^{13}$ Schlueter, E., Zimmerman, R.W., Myer, L.R., Cook, N.G.W., and Witherspoon, P.A. 1992c. Predicting the capillary pressure of Berea sandstone from microgeometry. Lawrence Berkeley Lab. Report in progress.

${ }^{14}$ Schultze, K. 1925a. Kolloid-Z 36,65.

${ }^{15}$ Schultze, K. 1925b. Kolloid-Z 37,10.

${ }^{16}$ Underwood, E.E. 1970. Quantitative Stereology. Addison-Wesley, Reading, MA.

${ }^{17}$ Yadav, G.D., Dullien, F.A.L., Chatzis, I., and Macdonald, I.F. 1987. Microscopic distribution of wetting and nonwetting phases in sandstones during immiscible displacements. SPE Reservoir Engineering, May, p.137-147.

${ }^{18}$ Yanuka, M., Dullien, F.A.L., and Elrick, D.E. 1986. Percolation processes and porous media: I. Geometrical and topological model of porous media using a threedimensional joint pore size distribution. J. Coll. Int. Sci. 112, p.24-41. 


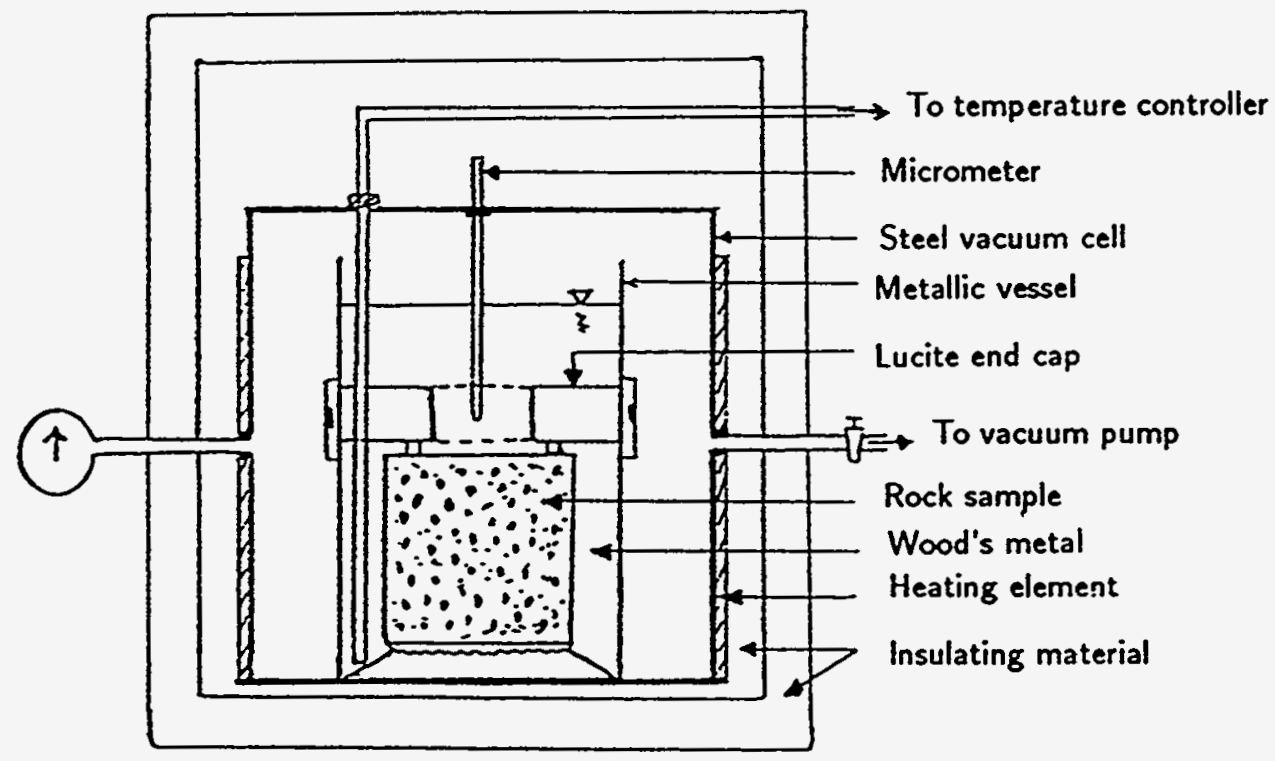

FIG. 7.1. Experimental setup for three-dimensional Wood's metal imbibition. 


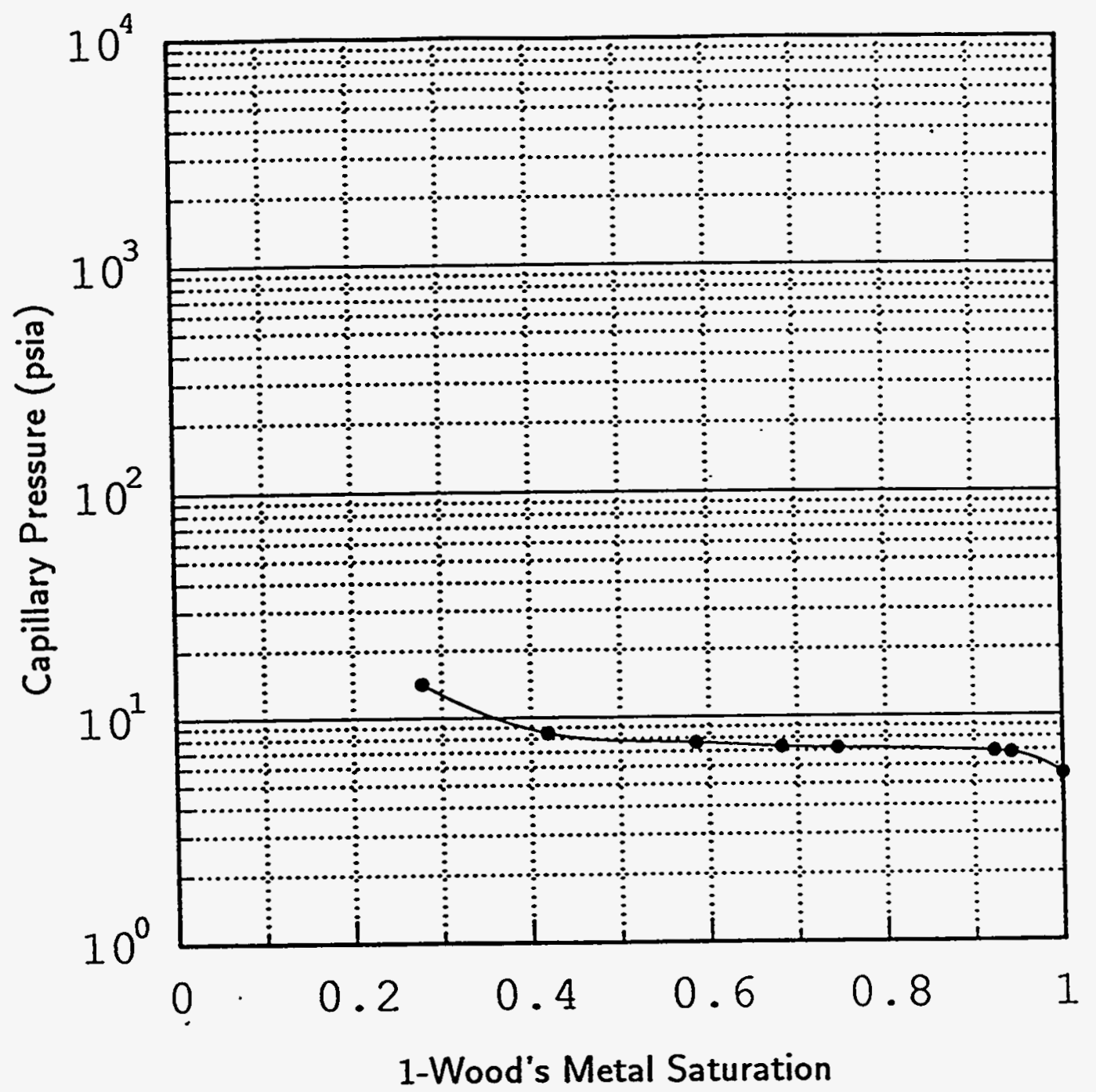

FIG. 7.2. Experimental capillary pressure function of Berea sandstone. The rock has been imbibed with a nonwetting fluid (Wood's metal) at different equilibrium pressures and solidified in place. The procedure allows for direct observation and analysis of the fluid distribution at a fixed pore pressure and saturation level. 


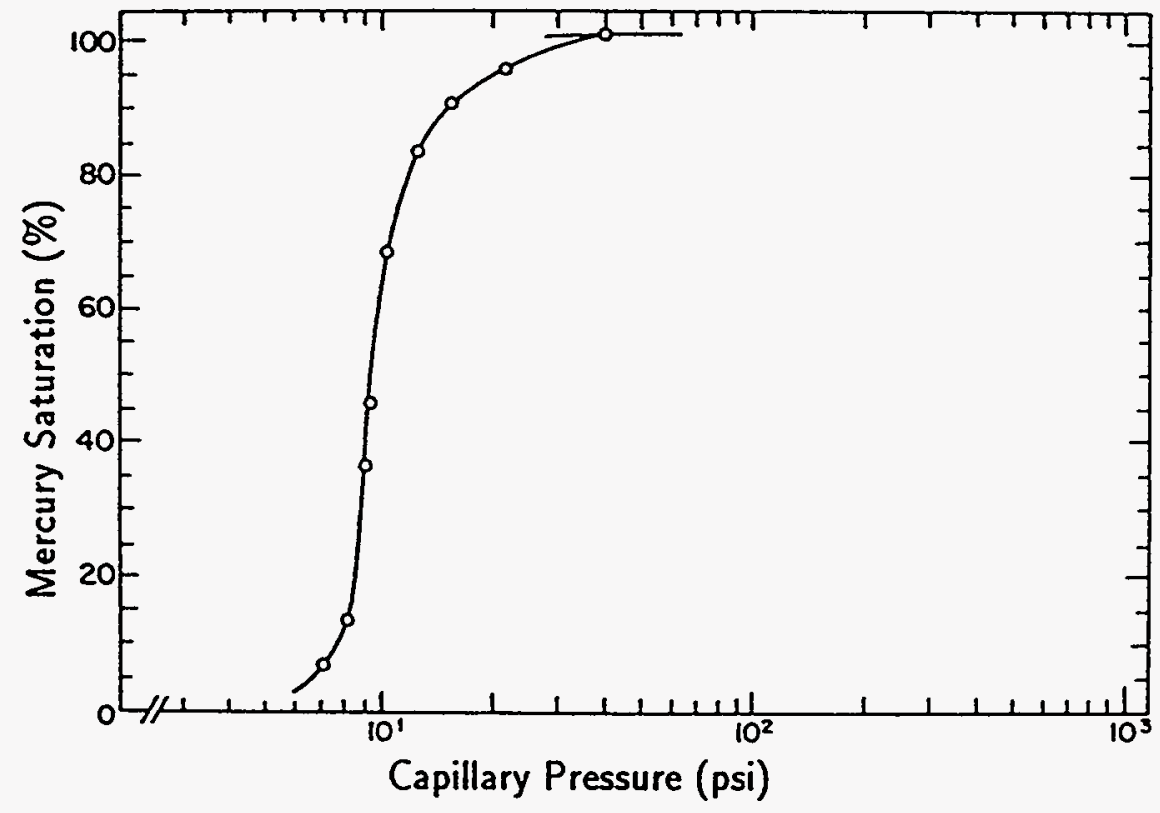

FIG. 7.3. Experimental mercury porosimetry saturation curve of Berea sandstone (after Chatzis and Dullien, 1977). 


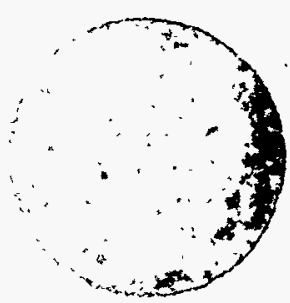

6.8 psia

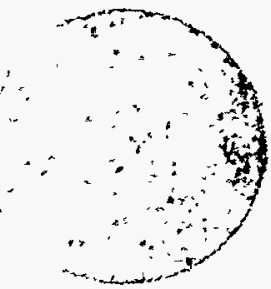

6.8 psia

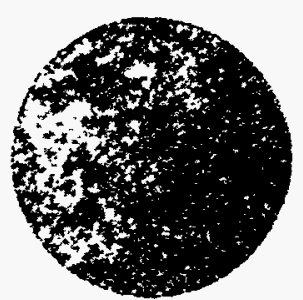

6.9 psia

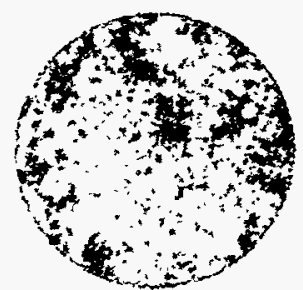

6.9 psia

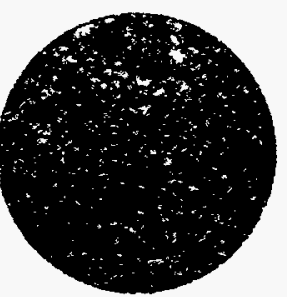

7.2 psia

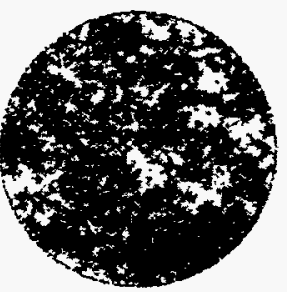

7.2 psia

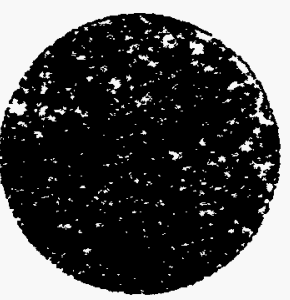

7.3 psia

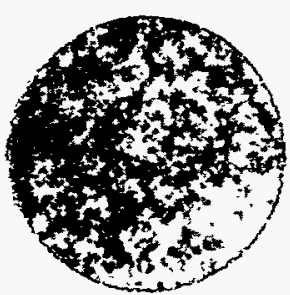

7.3 psia

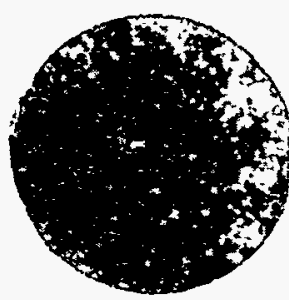

7.65 psia

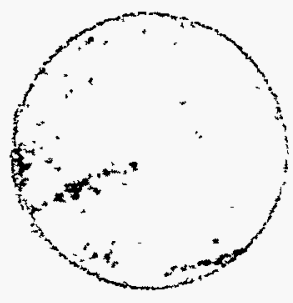

7.65 psia

FIG. 7.4. Top and bottom axial quarter sections of a Berea sandstone core partially saturated with a nonwetting fluid (Wood's metal) at different equilibrium pressures, and solidified in place. The procedure allows for direct observation and analysis of the fluid distribution at a fixed pore pressure and saturation level. The sections reveal that the fluid distributions are composed of a set of imbibing clusters correlated in space. 


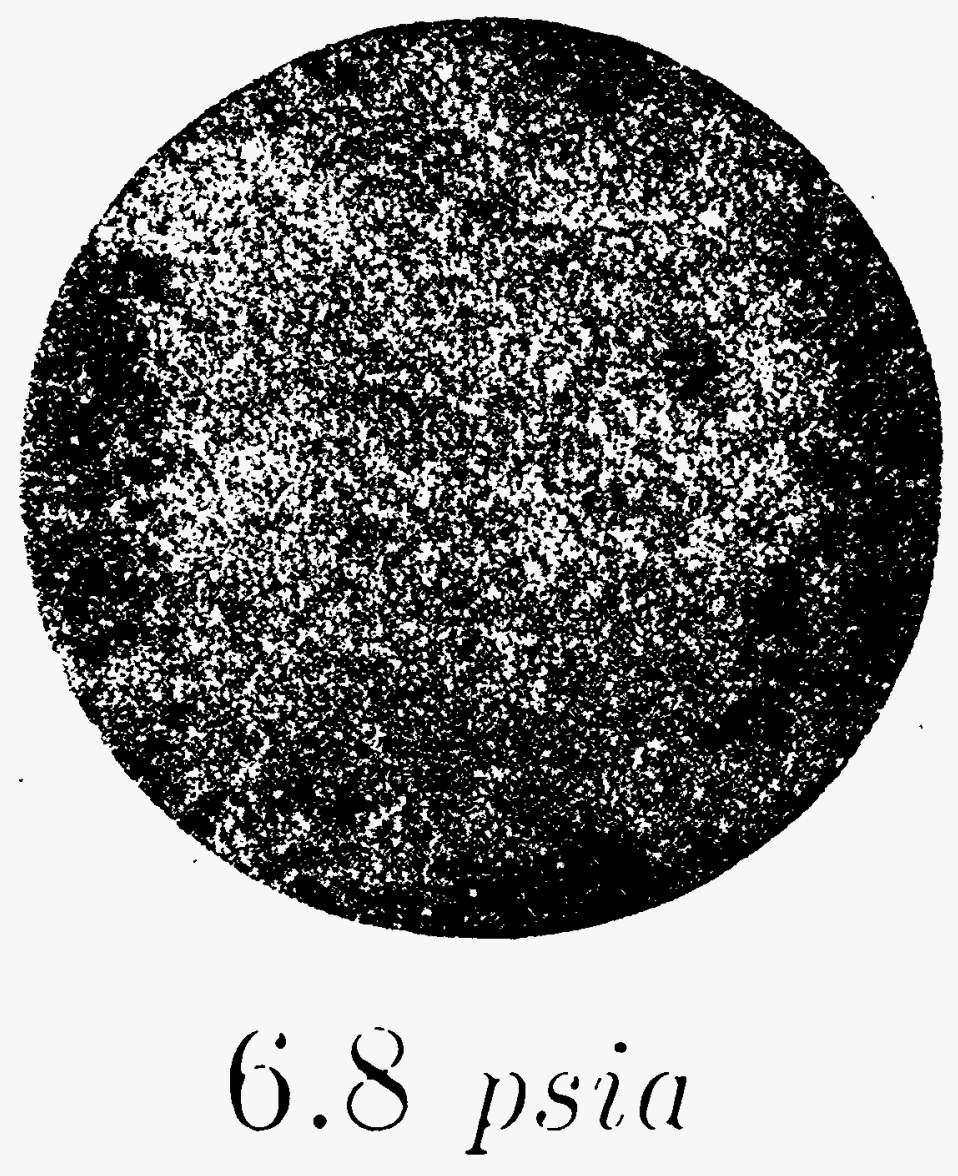

FIG. 7.5. Top section of a Berea sandstone core partially saturated with a nonwetting fluid (Wood's metal) at an equilibrium pressure of $6.8 \mathrm{psia}$, and solidified in place. The procedure allows for direct observation and analysis of the fluid distribution at this pressure and correspondent saturation level. 


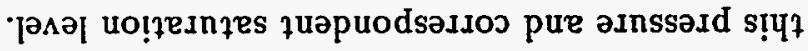

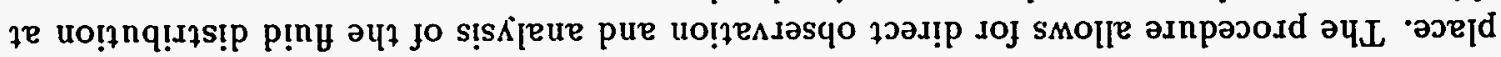

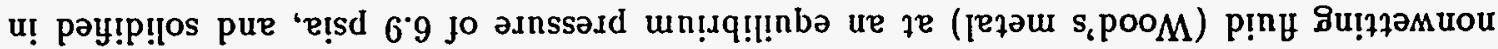

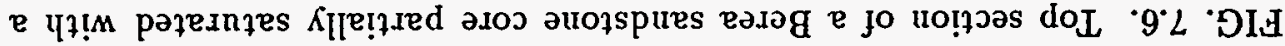

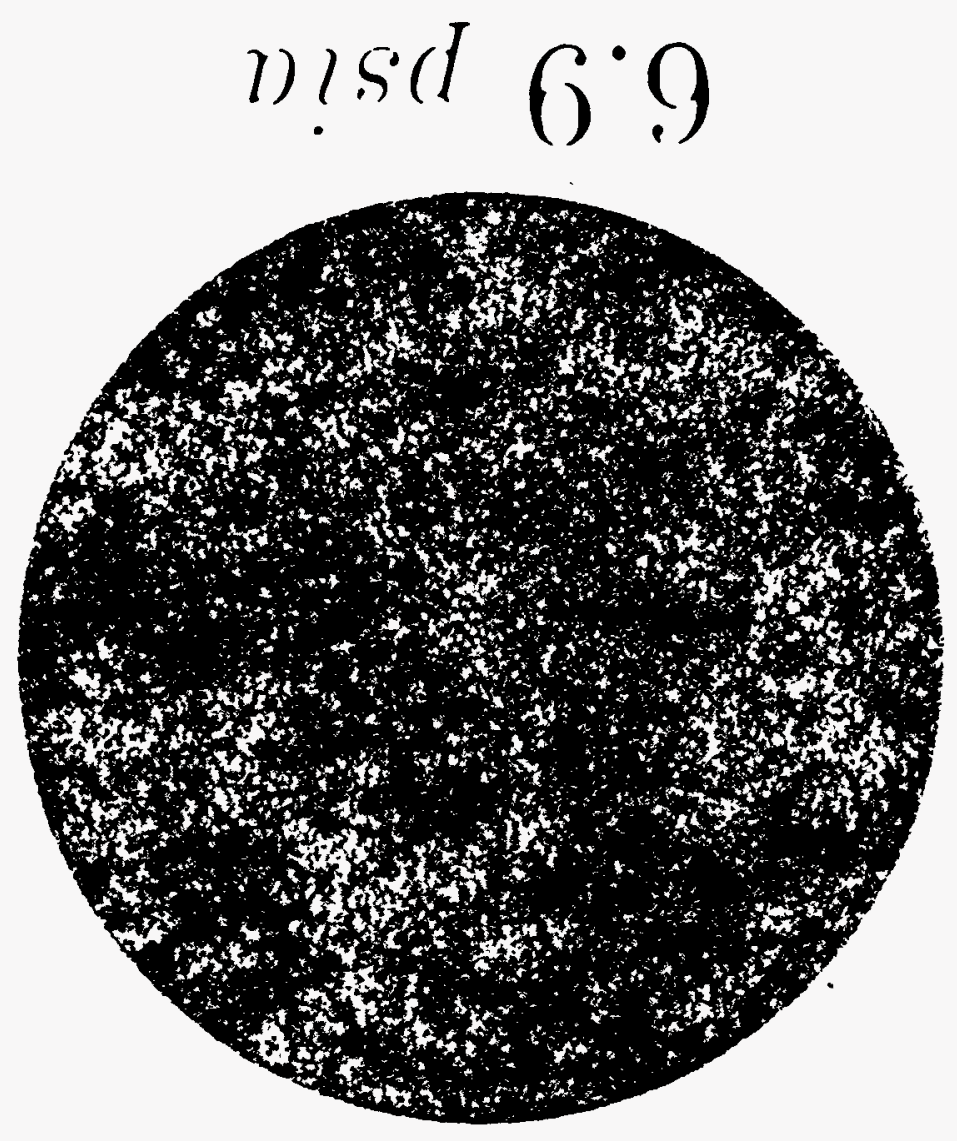




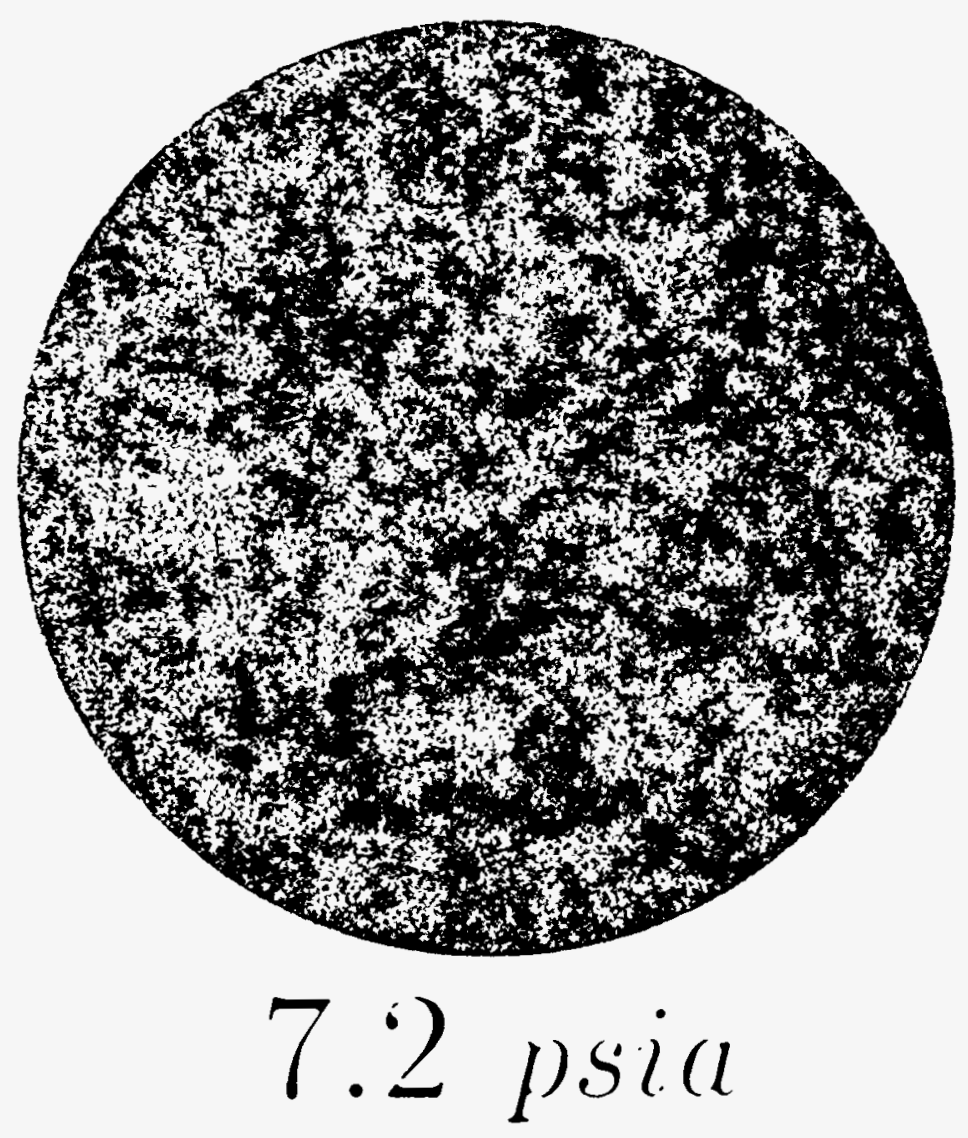

FIG. 7.7. Top section of a Berea sandstone core partially saturated with a nonwetting fluid (Wood's metal) at an equilibrium pressure of $7.2 \mathrm{psia}$, and solidified in place. The procedure allows for direct observation and analysis of the fluid distribution at this pressure and correspondent saturation level. 


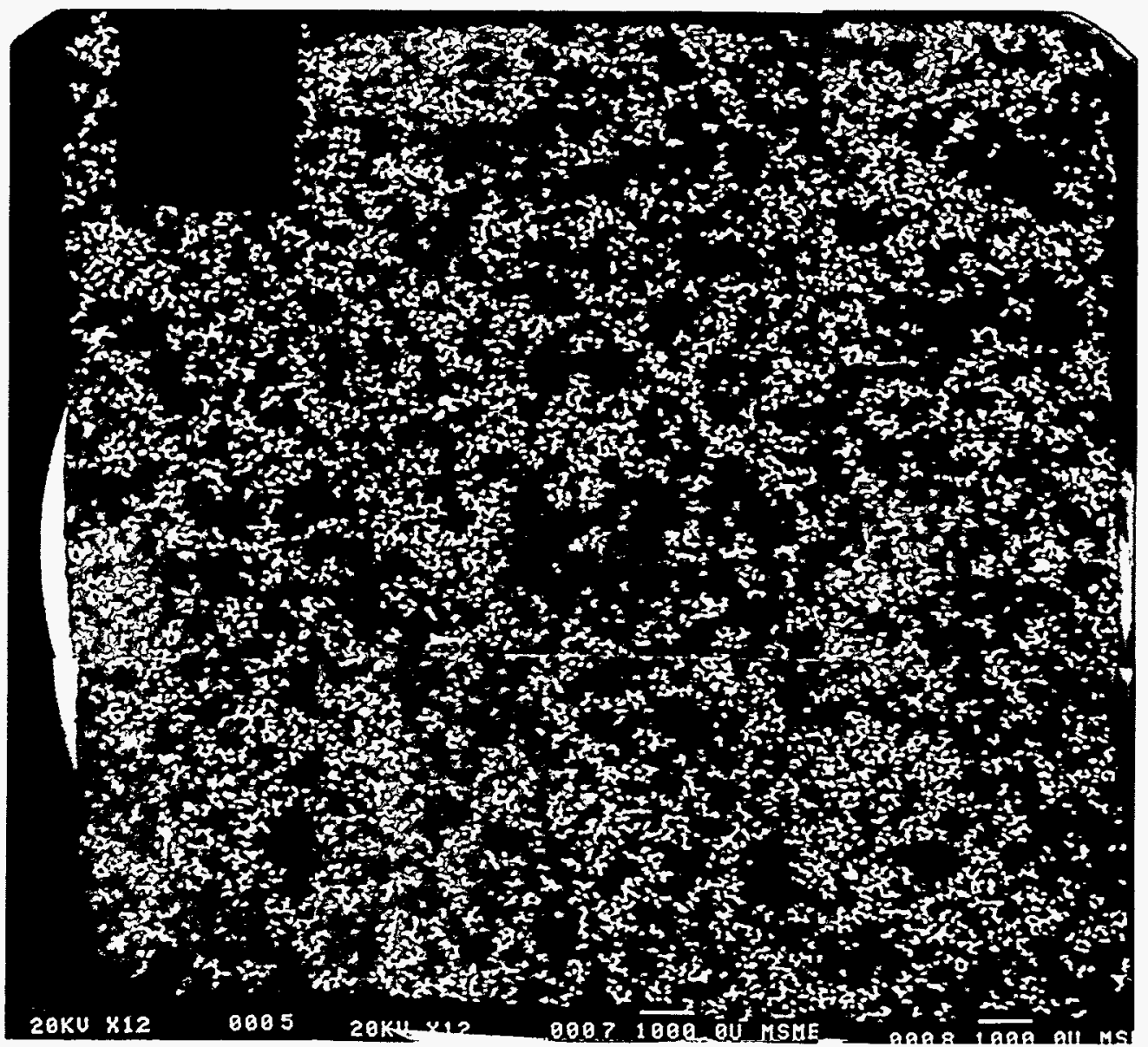

FIG. 7.8. Scanning electron microscope photomicrograph collage of a 1 in $\times 1$ in Berea sandstone specimen partially saturated with approximately $50 \%$ Wood's metal at 8.5 psia equilibrium pressure. The rock is composed mainly of quartz grains (gray phase), and pore space that has been impregnated with Wood's metal (white phase), and epoxy (black phase) for imaging purposes. 


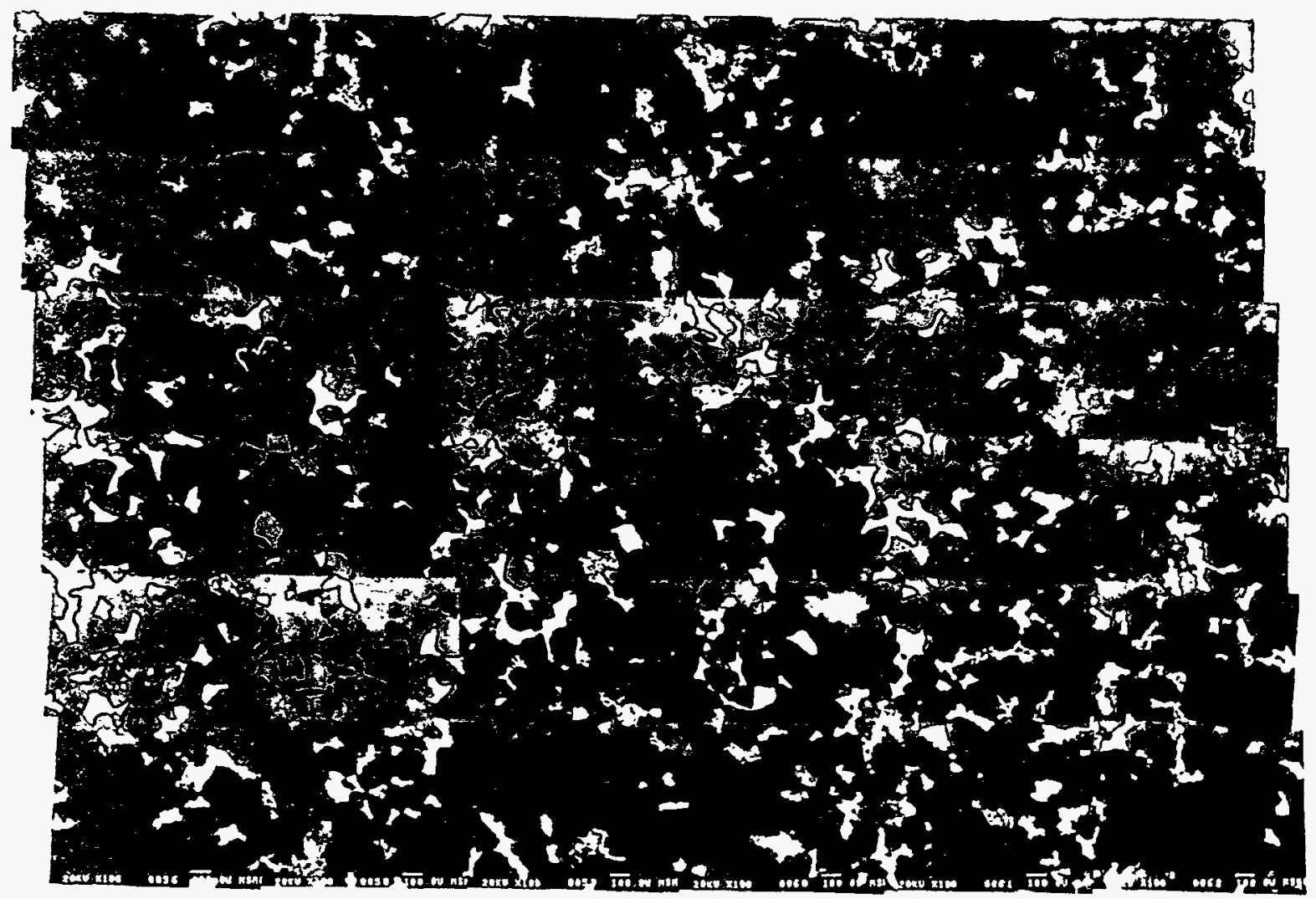

FIG. 7.9. Scanning electron microscope photomicrograph collage of an enlarged partial section obtained from a Berea sandstone sample partially saturated with approximately $50 \%$ Wood's metal (white phase) at 8.5 psia pressure shown in Fig. 7.8. Actual width of field is about $6 \mathrm{~mm}$. 


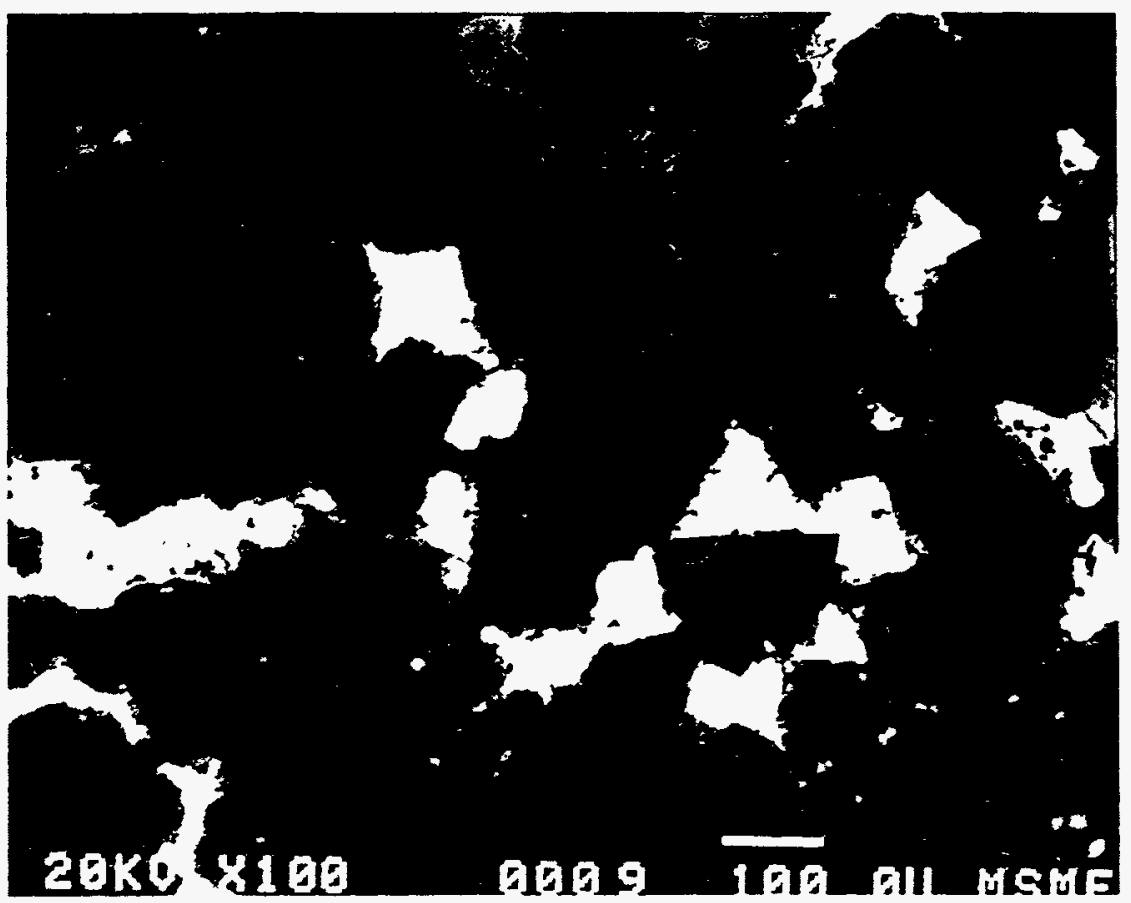

FIG. 7.10. Typical scanning electron microscope photomicrograph of Berea sandstone. The rock is composed mainly of quartz grains (dark-gray phase), feldspar grains (medium-gray phase), and products of grain dissolution (light-gray phase). The rock pore space has been impregnated with Wood's metal alloy (white phase), and epoxy (black phase). 


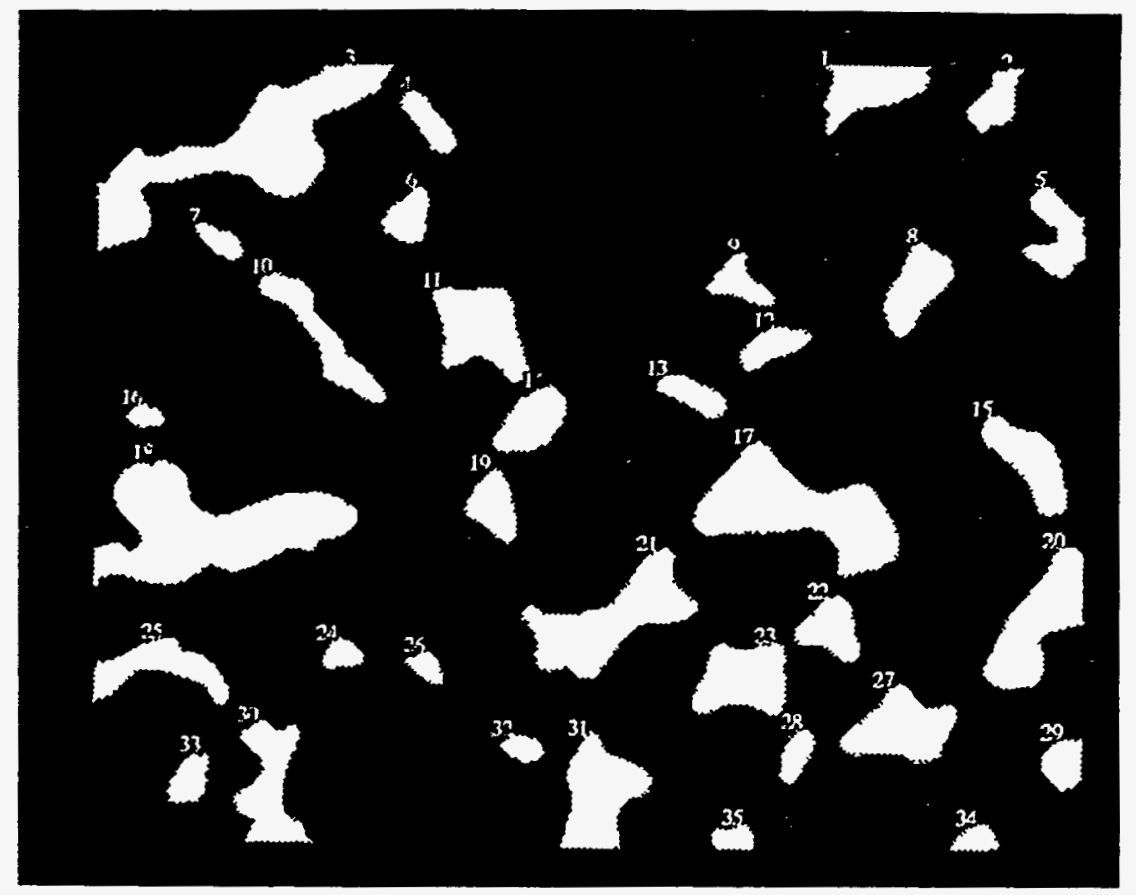

FIG. 7.11. Computerized analysis of Berea sandstone image shown in Fig. 7.10 display intergranular pore space contours from which hydraulic radius distribution and transport properties (hydraulic and electric) have been calculated. 


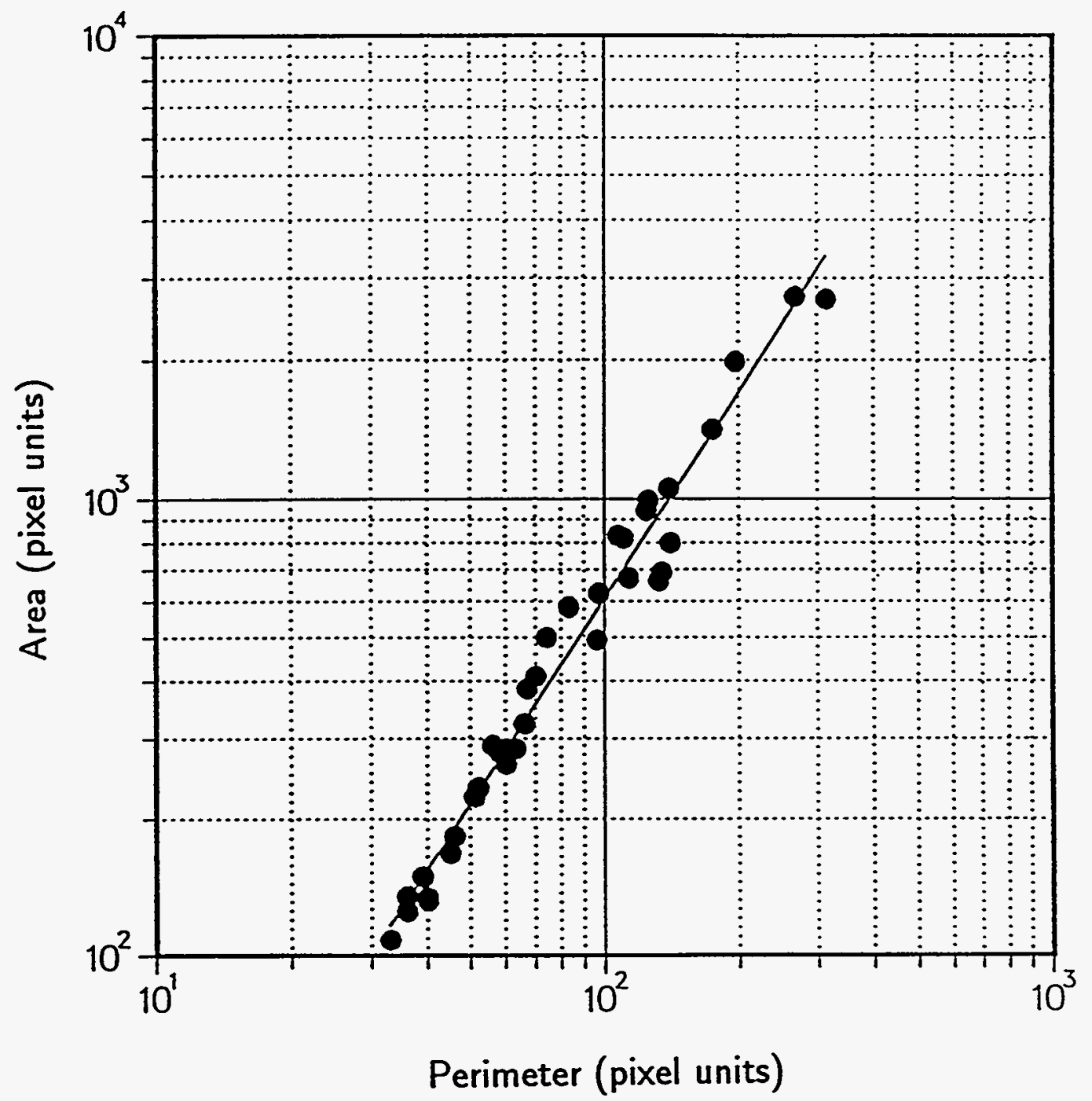

FIG. 7.12. Perimeter-area power-law relationship of Berea sandstone obtained from pore space contours shown in previous figure. 


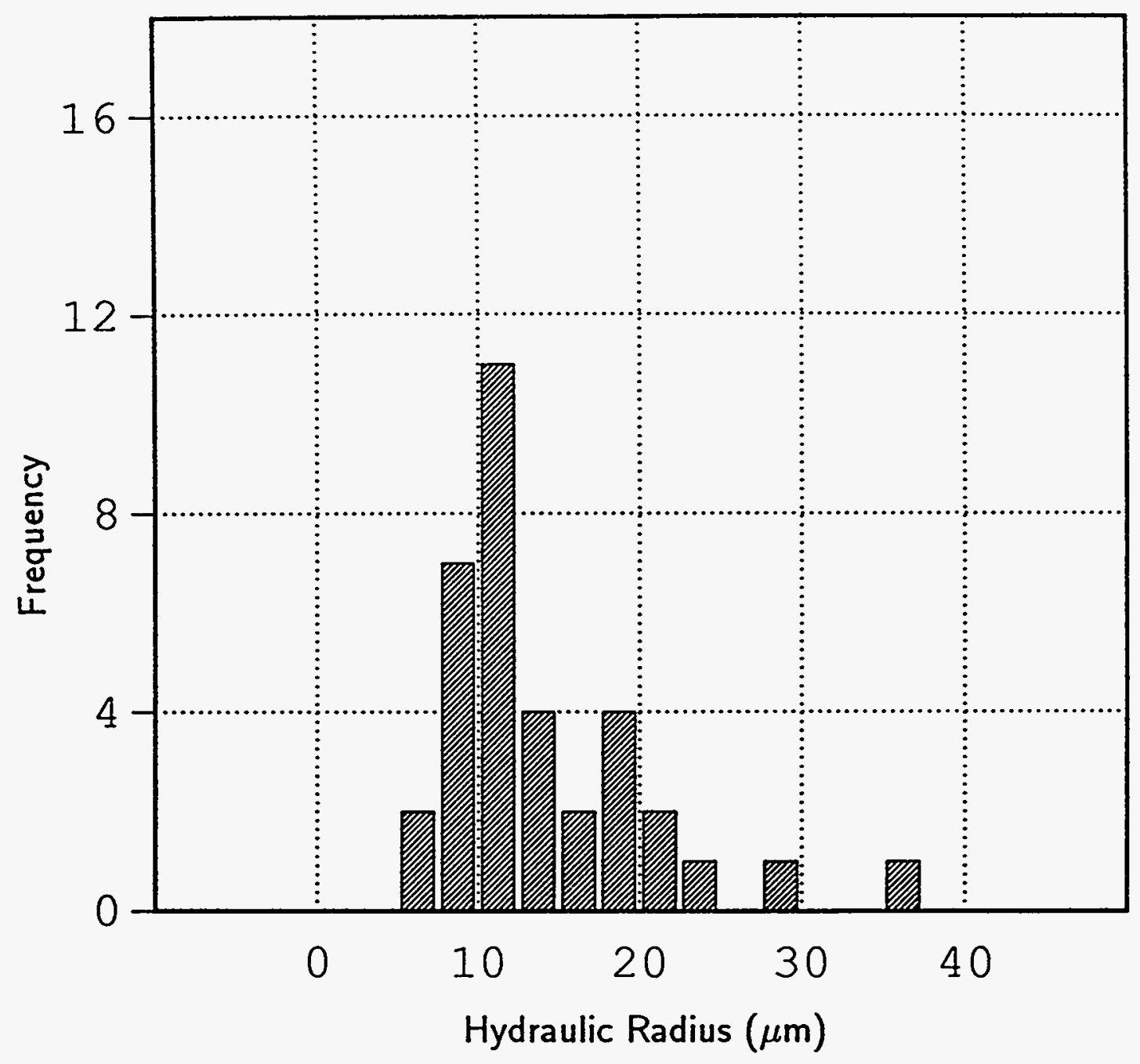

FIG. 7.13. Pore hydraulic radii frequency distribution of Berea sandstone obtained from pore space contours shown in Fig. 7.11. 


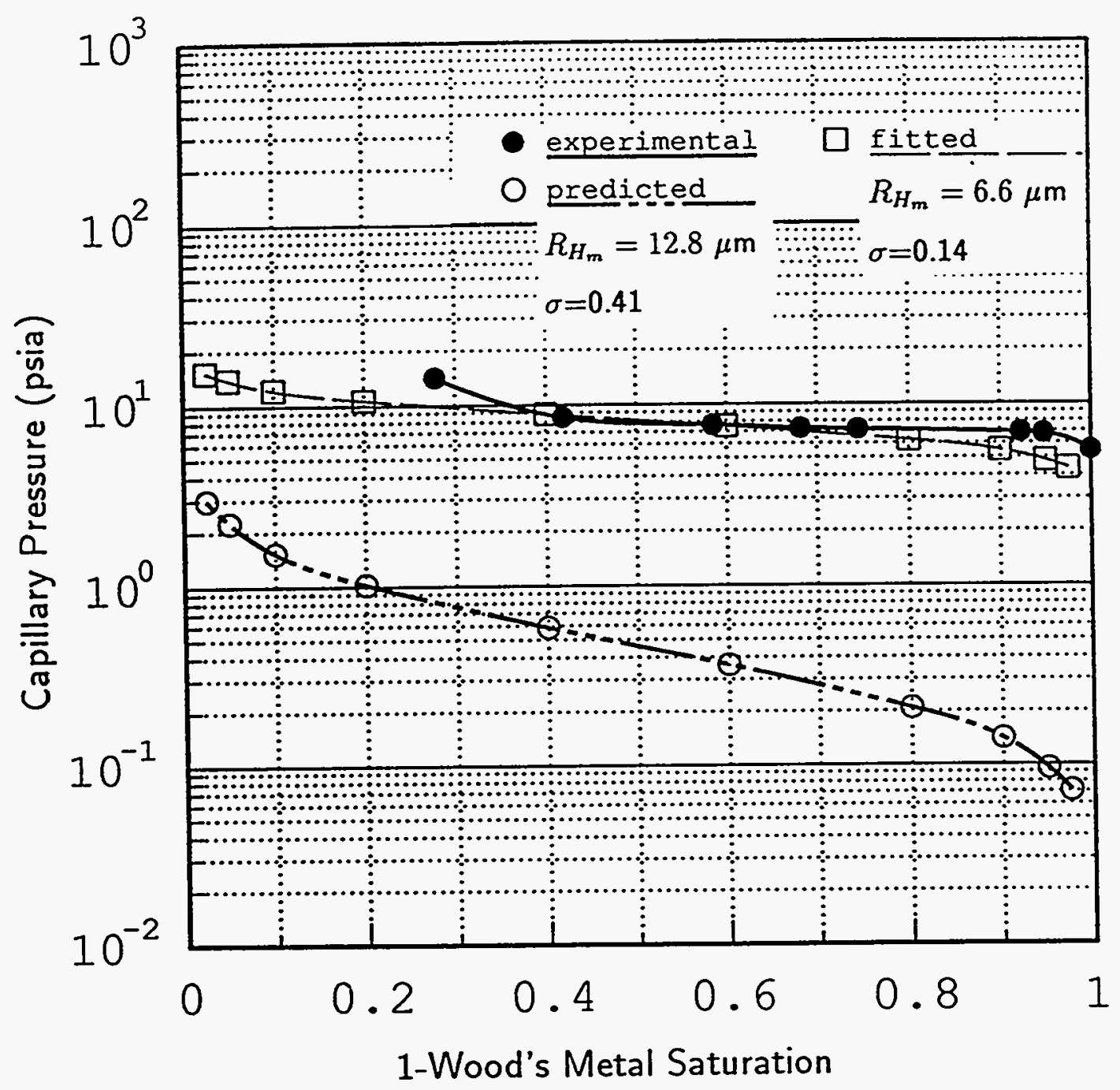

FIG. 7.14. Experimental vs. predicted capillary pressure function of Berea sandstone. To obtain the experimental capillary pressure function, the rock has been imbibed with a nonwetting fluid (Wood's metal) at different equilibrium pressures and solidified in place. The procedure allows for direct observation and analysis of the fluid distribution at a fixed pore pressure and saturation level. 


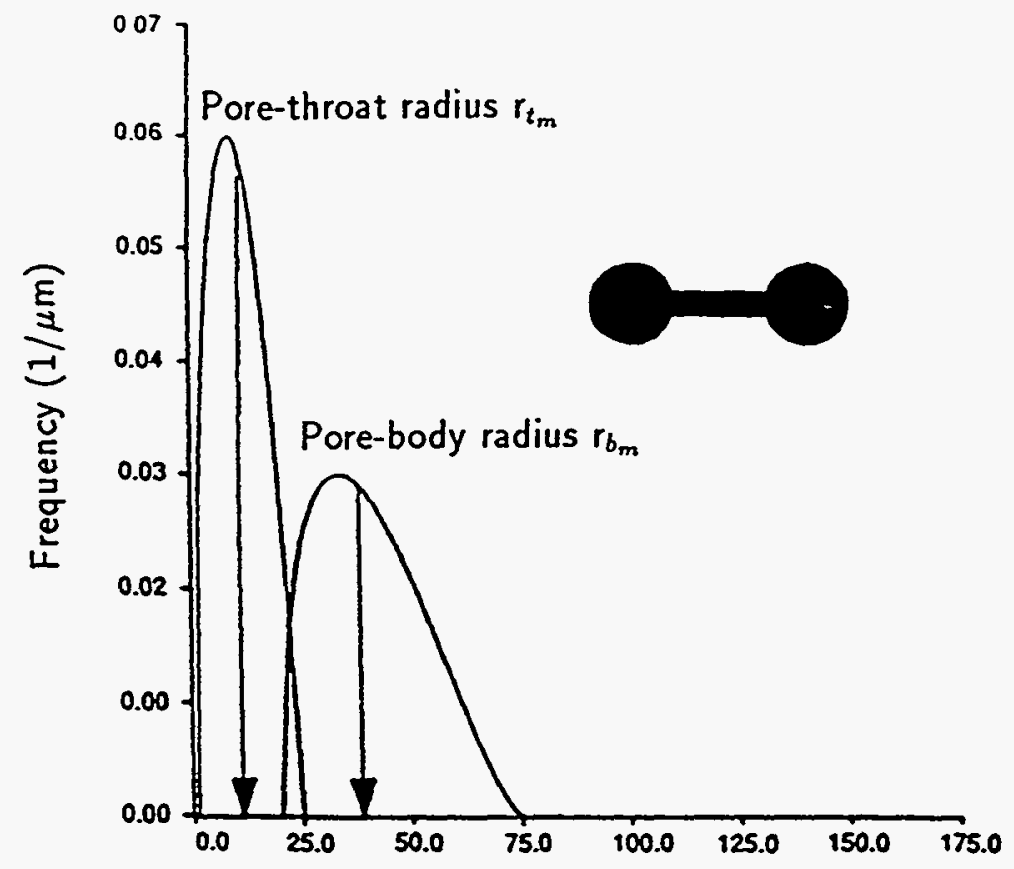

FIG. 7.15. Pore size distribution representative of a consolidated porous medium (Berea sandstone). Pore-throats are smaller than pore-bodies; $r_{t_{m}}=11 \mu \mathrm{m}$, $r_{b_{m}}=38 \mu \mathrm{m}$ (after Jerauld and Salter, 1990). 
TABLE 7.1. List of comparative values to show equivalence of the reciprocal hydraulic radius $\left(1 / R_{H}\right)$ and the reciprocal mean radius of curvature $\left(1 / r_{1}+1 / r_{2}\right)$ in a capillary $\left(r_{i}\right.$ is the radius of the inscribed circle) (after Carman, 1941).

\begin{tabular}{|c|c|c|}
\hline Cross-section & $1 / r_{1}+1 / r_{2}$ & $1 / R_{H}$ \\
\hline Circle & $2 / r$ & $2 / r$ \\
\hline Parallel plates & $1 / b$ & $1 / b$ \\
\hline Rectangle & $1 / a+1 / b$ & $1 / a+1 / b$ \\
\hline Equilateral triangle & $2 / r_{i}$ & $2 / r_{i}$ \\
\hline Square & $2 / r_{i}$ & $2 / r_{i}$ \\
\hline$a: b=2: 1$ & $1.50 / b$ & $1.54 / b$ \\
\hline Ellipse $\{a: b=5: 1$ & $1.20 j b$ & $1.34 / b$ \\
\hline$a: b=10: 1$ & $1.10 / b$ & $1.30 / b$ \\
\hline
\end{tabular}




\section{RECAPITULATION}

- We have attempted to assemble a comprehensive picture of a sedimentary rock based on observation of pore casts, laboratory measurements, and on a physically accurate representation of the phenomena.

- The analytical calculations are based on pore microgeometry measurements obtained directly from two-dimensional scanning electron microscope (SEM) photomicrographs of rock sections partially filled with wetting and nonwetting liquids solidified in place. Account has been taken of the fact that the rock crosssections are randomly oriented with respect to the channel axes. The orientation effect is corrected by means of geometrical and stereological considerations. In addition, account is also taken of possible variation of the cross-sectional area along the length of each tube, e.g., pore-throats and pore-bodies.

- Good agreement was found between measured and predicted analytical calculations of permeability for a variety of sandstones using the cubic lattice-effective medium model of intergranular pore space (discrete approach). It is found that the ratio of average pore-throat to pore-body ranges from about 0.3 to 0.5 for different sandstones.

- If a parallel-effective medium or series model is used instead, permeability is overpredicted or underpredicted, respectively, by at least a factor of three. This investigation highlights the importance of pore connectivity in understanding the relationships between pore microstructure and permeability.

- A perimeter-area power-law relationship of a smooth representation of the pore space was found for a variety of rocks of different porosities.

- Examples have been given of how the area-perimeter power-law information together with a pore-size distribution and a classical model of the pore space can be used for making quantitative predictions of permeability (statistical approach). The analysis emphasizes the importance of pore connectivity in understanding the relationship of permeability and rock microstructure.

- Fair to good agreement was found between measured and predicted electrical conductivities, for a variety of sandstones when using the cubic lattice-effective medium model of the intergranular pore space. In particular, good agreement was found in case of a poorly consolidated rock such as Saint-Gilles sandstone, whereas the agreement was not very good for well-cemented rocks. The possible reason(s) for this effect were empirically investigated. 
- In our experimental studies we have employed wetting and nonwetting phases that can be frozen in place one at a time; to allow measurement of effective transport properties (hydraulic and electric); and to allow direct analysis of fluid distributions in the pore space after the experiment.

- At low wetting phase saturations in Berea sandstone pore space (saturations less than $30 \%$ ), the wetting fluid invades preferentially grain contact pore space (e.g., thin sheets and micropores) while only coating intergranular pore space connected by larger throats. We observe a substantial effect on effective formation factor (but a very small effect on effective permeability).

- It is observed that there is no unique definition of pore structure and pore-size distribution to model both permeability and formation factor, e.g., permeability is strongly controlled by intergranular pore space whereas the formation factor is controlled by intergranular and grain contact pore spaces.

- The basic intergranular pore space model that was employed in the analytical calculations of formation factor has to be modified to incorporate relevant geometrical and topological characteristics important for electrical conductivity of consolidated rocks (e.g., grain contact pore space).

- It is found that there is no single characterization of the Archie saturation exponent, $\mathrm{n}$, for the full range of saturation. Archie saturation exponents are found to vary from about 3 when mainly grain contact pore space is filled with the wetting fluid to about 5 when mainly intergranular conduits connected by the larger throats are filled with the wetting fluid, with a critical electrolyte saturation $\left(S_{\text {crit }}\right)$ of 0.7 .

- Eventhough the change in trend of Berea sandstone electrical conductivity at low electrolyte concentrations reflects the contribution of surface conduction due to clays, this represents a negligible contribution to formation factor for most practical applications.

- Pore microgeometry based analytical calculations of the capillary pressure vs. saturation function have been conducted. The mathematical expression depends on the distribution of pore hydraulic radii and the area-perimeter power-law relationship. The analytical predictions of capillary pressure are compared to laboratory capillary pressure curves obtainead using Wood's metal alloy as the nonwetting phase intead of the conventional mercury porosimetry. This technique allows the examination of the occupied pore space after the experiment. 
- The general shape of our experimental capillary pressure vs. saturation curve for Berea sandstone is consistent with typical capillary pressure curves reported in the literature obtained using mercury porosimetry.

- The nonwetting fluid in Berea sandstone (in the saturation range up to 50\%) invades and fills relatively few intergranular conduits connected by large throats, producing a clustered structure, and controlling capillary pressure within that saturation range.

- The analytical calculations of Berea sandstone capillary pressure vs. saturation relation together with the analysis of the nonwetting phase fluid distributions in the rock pore space show that the experimental capillary pressure vs. saturation function (in the saturation range up to about $50 \%$ saturation) is controlled by relatively few channels connected by large intergranular throats of narrow size distribution, represented by a mean hydraulic radius of approximately $6.6 \mu \mathrm{m}$ and a standard deviation of 0.14 .

- At low wetting phase saturations in Berea sandstone pore space, the wetting fluid invades preferentially grain contact pore space, coats intergranular pore space connected by larger throats, permitting the nonwetting fluid to rapidly invade relatively few intergranular conduits connected by large throats, controlling capillary pressure (and relative permeability). Thus, pore geometry and topology establish a hierarchy that governs how wetting and nonwetting fluid invasion processes happen in the pore space, allowing the two processes to be complementary, e.g., each fluid follows its own path.

- It is clear from this study that the transport properties of porous media (hydraulic and electric) are strongly controlled by pore microgeometry and topology.

\section{EXTENSIONS}

- Analyze experimental relative permeability data of Berea sandstone in light of the distribution of wetting and nonwetting phases in the rock pore space.

- Examine experimental relative permeability data of Berea sandstone near threshold saturation conditions.

- Develop constitutive relations between relative permeability, effective formation factor, and saturation for Berea sandstone. 
- Employ network/percolation model to predict relative permeability of Berea sandstone from microgeometry.

- Study the relationship between area-perimeter power-law relationship of pores and relative permeability.

- Evaluate rock void space power-law relationships (e.g., perimeter-area, volumesurface) further using fractal geometry.

- Apply experimental method and analysis to study single and two-phase transport properties (hydraulic and electric) of unconsolidated/partially consolidated porous media to be compared to consolidated porous media.

- Apply experimental method and analysis to study three-phase transport properties of consolidated/partially consolidated porous media.

- Apply experimental method and analysis to study single and multiphase transport properties of fractured media.

\section{ACKNOWLEDGMENTS}

This work was supported by the U.S. Department of Energy through the Assistant Secretary for Fossil Energy, Bartlesville Project Office, Advanced Extraction Process Technology (AEPT), under contract No. DE-AC22-89BC14475, with the University of California at Berkeley. Additional funding was provided by the Director, Office of Energy Research, Office of Basic Energy Sciences, Engineering and Geosciences Division, under Contract No. DE-AC03-76SF00098 with the Lawrence Berkeley Laboratory. I am happy to acknowledge technical guidance from Dr. R.W. Zimmerman, Dr. L.R. Myer, Dr. K. Pruess, and Dr. G. Sposito. Heartfelt thanks to my professors and academic advisers Dr. P.A. Witherspoon, and Dr. N.G.W. Cook. We thank Dr. R. Suarez-Rivera for providing Berea sandstone acid-treated sample KS11, and Dr. P. Persoff for providing Boise and Saint-Gilles sandstone samples. Thanks are also due to R. Curtis of Lawrence Berkeley Laboratory for technical assistance with the experimental equipment, and to R. Wilson of U.C. Berkeley for expertise with the scanning electron microscope. 